
upf.
Universitat Department
Pompeu Fabra of Economics and Business
Barcelona

Economics Working Paper Series

Working Paper No. 1639

\title{
The effects of conventional and unconventional monetary policy on exchange rates
}

Atsushi Inoue and Barbara Rossi

December 2018 


\title{
The Effects of Conventional and Unconventional Monetary Policy on Exchange Rates
}

\author{
Atsushi Inoue $^{\dagger} \quad$ Barbara Rossi* \\ Vanderbilt University ICREA-Univ. Pompeu Fabra, \\ Barcelona GSE, and CREI
}

This Draft: December 8, 2018

\begin{abstract}
What are the effects of monetary policy on exchange rates? And have unconventional monetary policies changed the way monetary policy is transmitted to international financial markets? According to conventional wisdom, expansionary monetary policy shocks in a country lead to that country's currency depreciation. We revisit the conventional wisdom during both conventional and unconventional monetary policy periods in the US by using a novel identification procedure that defines monetary policy shocks as changes in the whole yield curve due to unanticipated monetary policy moves and allows monetary policy shocks to differ depending on how they affect agents' expectations about the future path of interest rates as well as their perceived effects on the riskiness/uncertainty in the economy. Our empirical results show that: (i) a monetary policy easing leads to a depreciation of the country's spot nominal exchange rate in both conventional and unconventional periods; (ii) however, there is substantial heterogeneity in monetary policy shocks over time and their effects depend on the way they affect agents' expectations; (iii) we find favorable evidence to Dornbusch's (1976) overshooting hypothesis; (iv) changes in expected real interest rates play an important role in the transmission of monetary policy shocks.
\end{abstract}

*Corresponding author: Barbara Rossi, Universitat Pompeu Fabra, Barcelona GSE ${ }^{\ddagger}$ and CREI, C. Trias Fargas 25-27, 08005 Barcelona, Spain. E-mail: barbara.rossi@upf.edu 
${ }^{\dagger}$ Department of Economics, Vanderbilt University, VU Station B, Box \#351819, 2301 Vanderbilt Place, Nashville, TN 37235, USA. E-mail: atsushi.inoue@vanderbilt.edu

Acknowledgments. Supported by the Fundación BBVA scientific research grant (PR16_DAT_0043) on Analysis of Big Data in Economics and Empirical Applications and the Cerca Programme/Generalitat de Catalunya. We thank Charles Engel, Jordi Gali', Refet Gürkaynak, James Morley, Giovanni Ricco, Jonathan Wright, seminar participants at the 2018 ISoM conference, 2018 ASSA, UCL, Tor Vergata, Bank of Russia, the 2018 Royal Economic Society conference as well as Lukas Hoesch for excellent research assistance and Silvia Miranda-Agrippino for help with the UK data. ${ }^{\ddagger}$ The Barcelona GSE acknowledges financial support from the Spanish ministry of the Economy and Competitiveness through the Severo Ochoa Programme for Centers of Excellence in R\&D (SEV2015-0563).

J.E.L. Codes: F31, F37, C22, C53. Keywords: Exchange rates, Zero-lower bound, Unconventional monetary policy, Forward guidance. 


\section{Introduction}

Central banks have recently been forced to rely on unconventional monetary policies due to the ineffectiveness of conventional policies at the zero lower bound. The unconventional policies include altering the size and composition of Central banks' balance sheets (i.e. Large Scale Asset Purchases programs, or LSAP) and/or issuing announcements about the future path of short-term interest rates (i.e. forward guidance). Have these new policies affected the way monetary policy shocks are transmitted to international financial markets, in particular exchange rates? And do the effects differ depending on how monetary policy affects agents' expectations regarding the future path of interest rates? Regarding the first question, several studies have found that conventional, expansionary monetary policies typically depreciate the exchange rate of the country implementing such policies (see e.g. Clarida and Gali', 1994, Eichenbaum and Evans, 1996, among others). However, during the recent decade, the implementation of unconventional monetary policy has become more and more frequent: whether the way monetary policy affects international financial markets has changed as well is an open question. Furthermore, regarding the second question, monetary policy shocks are typically identified in the literature as unexpected changes in short-term interest rates that are exogenous to the state of the economy (cfr. Eichenbaum and Evans, 1996). However, monetary policy may have other dimensions, both in the conventional and in the unconventional period, as its effects may depend on how it affects agents' perception of future expected monetary policy, riskiness and uncertainty in the economy. For example, Gürkaynak, Sack and Swanson (2005a) find that monetary policy announcements have important effects on the term structure of interest rates even if the short-term interest rate did not change.

To answer these questions, we use a new approach to the identification of monetary policy shocks, where shocks are defined as shifts in the entire term structure of interest rates on a day of a monetary policy announcement. Our framework differs from the traditional literature since it naturally captures alternative dimensions of monetary policy (such as forward guidance and asset purchases programs announcements) embedded in shifts of the whole term structure triggered by unexpected monetary policy moves. The approach is 
inspired by Inoue and Rossi's (2017) Functional VARs, although we considerably depart from it by using a non-parametric approach without taking a stand on the specification of term structure models. Relative to using factor models to parameterize the term structure, using a selection of raw yields has the following advantages: first, it is a non-parametric approach and thus does not require taking a stand on the parametric model. Second, it allows us to be robust to the possibility that the number of factors that are relevant to explain exchange rate fluctuations may change over time, or that additional factors might be important in specific episodes. Although it is quite common to model the yield curve using three factors, there is some empirical evidence for a fourth factor (Cochrane and Piazzesi, 2009; Dewachter and Lyrio, 2006; and De Pooter, 2007). Furthermore, there is some evidence that the number of factors in conventional times is two while it becomes three in unconventional times (Swanson, 2017). Our approach is robust to the presence of additional factors, and even if an additional factor appears sporadically or only in part of the sample. Third, because the cross sectional dimension of yield curve data is small, estimating models with factors introduces sampling error. By using the individual yield data directly, we do not need to take into account the sampling error. On the other hand, our approach can be implemented using a parametric model (such as a factor model) instead of the raw yields.

In Section 6, we distinguish among several types of announcements and link them to the shape of the term structure changes. We view our shock as a parsimonious way of simultaneously capturing the overall unexpected monetary policy event; it might also be interesting to map the shape of the term structure changes to the specific words in the announcement. In order to see how the yield curve moves in response to specific monetary policy words or statements, a more detailed textual analysis is needed but it is outside the scope of the paper.

By examining the exchange rates of the UK, Europe, Canada and Japan vis-a'-vis the US dollar, we find that a country's monetary policy tightening in the conventional period generally leads to an appreciation of that country's nominal spot exchange rate, a result consistent with Clarida and Gali' (1994), Eichenbaum and Evans (1996) and Faust and Rogers (2003). However, interestingly, the effects on exchange rates differ depending on how monetary pol- 
icy affects agents' expectations as well as its perceived effects on the riskiness/uncertainty in the economy in specific episodes. In particular, on average across episodes, the appreciation (depreciation) that follows a contractionary (expansionary) monetary policy shock is mostly due to changes in expectations in the short-run, although changes in medium to long-term expectations turn out to be important in selected episodes. The possibility that monetary policy might be multi-dimensional was first discussed and empirically investigated in the seminal work by Gürkaynak, Sack and Swanson (2005a). In this paper we take their analysis a step further: in fact, our approach can be viewed as a way to systematically capture all the various dimensions in which monetary policy affects international financial markets via changes in agents' expectations and perception of risk/uncertainty in the economy, and their time variation.

At our daily frequency, we also find empirical evidence in favor of Dornbusch's (1976) overshooting hypothesis. This result is considerably different from similar analyses, based on monthly or quarterly data, that typically find that the US dollar continues to appreciate for a substantial period of time after a US contractionary monetary policy shock (e.g. Eichenbaum and Evans, 1995).

Since the definition of the monetary policy shock is the same no matter whether monetary policy is conventional or unconventional, we can consistently compare the effects of monetary policy in the two regimes. The effects of unconventional monetary policy on spot exchange rates are qualitatively similar to those in conventional times; hence, monetary policy did not lose its effectiveness in unconventional times. However, the exchange rate depreciation following an unconventional monetary policy easing is mostly due to changes in expectations in the medium- to long-run.

Finally, we use real exchange rates to shed light on the channels through which monetary policy operates. Exogenous movements in expected nominal interest rates should be reflected in changes in nominal exchange rates. However, changes in expected nominal interest rate might be due to either changes in future expected real interest rates or changes in future expected inflation. In order to investigate whether the transmission channel is indeed via changes in real interest rates, we focus on real exchange rates. If the channel through which 
monetary policy shocks affect real exchange rates is via changes in inflation expectations, we should not see any effects of monetary policy on real exchange rates. We find that, in the unconventional period, a real, expansionary US monetary policy shock depreciates the US dollar and a contractionary one appreciates it, even at the long end of the yield curve. Hence, this rules out that the transmission mechanism operates only via expected inflation, and suggests that expected medium- and long-run changes in real interest rates triggered by monetary policy play an important role. Given the high correlation between nominal and real exchange rates at daily frequencies, this suggests that also nominal exchange rate fluctuations are driven by monetary policy shocks via movements in real interest rates differentials.

Our work is related to the vast literature that studies the effects of monetary policy on exchange rates. It is well-known that expansionary shocks typically lead to a depreciation of the currency - see Clarida and Gali', 1994; Eichenbaum and Evans, 1996; Faust and Rogers, 2003; Scholl and Uhlig, 2008; Bouakez and Normandin, 2010, among others. However, the latter papers focus on the conventional monetary policy period, where monetary policy shocks can be identified as exogenous changes in short-term interest rates; the effects of unconventional monetary policy shocks, instead, are relatively less studied. Recent papers that focus on the unconventional period are Rogers, Scotti and Wright (2014, 2016) and Glick and Leduc (2015). As unconventional monetary policies are a combination of asset purchases and forward guidance, they estimate monetary policy surprises in a short window of time around monetary policy announcements. Rogers, Scotti and Wright (2014) study the effects of monetary policy shocks identified in two principal components extracted from a cross-section of yields on bond yields, stock prices and exchange rates for the US, UK, Euroarea and Japan. Rogers, Scotti and Wright (2016) estimate the effects of unconventional monetary policy surprises on both excess returns on carry trade portfolios as well as a variety of macroeconomic variables (bond yields, exchange rates, employment, inflation and interest rate spreads) and foreign risk premia in a VAR with external instruments. Glick and Leduc (2015) distinguish between changes in the Fed Funds Rate (FFR) around monetary policy announcements; changes in the one-year ahead euro-dollar future rate (short-run path surprises); and changes in the first principal component from several long-term Treasury 
rate futures (long-run path surprises). They find that monetary policy is effective in both conventional and unconventional periods. Also, in the conventional period, the U.S. dollar depreciates in response to a short-term easing but not to a long-term one; on the contrary, in the unconventional period, the U.S. dollar depreciates in response to both short-term and long-term path surprises. Our paper differs from these contributions in several ways. A first difference is that, in the latter papers, the shock is the exogenous change in the principal component(s) extracted from a cross section of interest rates, while in our work the shock is the shift in the entire term structure due to an exogenous monetary policy move. It is the analysis of how the whole yield curve shifts over time that allows us to crucially differentiate our results from those existing in the literature. In fact, we use an alternative measure of monetary policy shocks that allows shocks to potentially differ in each monetary policy episode depending on how the shock is perceived by the agents at different horizons. A second, important difference is that Rogers, Scotti and Wright (2014) and Glick and Leduc (2015) use an event study approach which allows them to estimate the contemporaneous correlation between changes in the term structure due to monetary policy on specific dates and the exchange rate, but is otherwise silent on the dynamic effects; in contrast, our paper estimates the whole dynamic impulse response. Rogers, Scotti and Wright $(2014,2016)$ also complement their analyses with VARs using either heteroskedasticity-based identification (as in Rogers, Scotti and Wright, 2014, and Wright, 2012), or external instruments (as in Rogers, Scotti and Wright, 2016) to trace out the effects of monetary policy shocks over time. Our approach instead relies on the Functional VAR approach (Inoue and Rossi, 2017a), which provides the dynamic response to the shift in the whole term structure. Finally, our analysis naturally leads to time-varying responses of exchange rates that fundamentally depend on the ways in which monetary policy affects agents' expectations of current and future interest rates as well as the risk and uncertainty in the economy. Our results that the effects of monetary policy on exchange rates are similar in the conventional and unconventional periods are consistent with Neely (2015).

In a related paper, Gali' (2018) analyzes the effectiveness of forward guidance in open economies. According to economic theory, under standard economic assumptions, the im- 
pact of an announcement of a future adjustment in interest rates on the current exchange rate either does not depend on the timing of the adjustment or it is larger the longer the horizon of implementation, depending on whether prices are assumed to be fixed or flexible. Empirically, however, Gali' (2018) finds instead that expectations of interest rate differentials in the near (distant) future have larger (smaller) effects than implied by theory. Since the theory is inconsistent with the empirical results, he concludes that there is a forward guidance exchange rate puzzle. In this paper, instead, we focus on the overall response of exchange rates to a monetary policy "event", which is defined as the shift in the entire term structure around monetary policy announcement dates, as opposed to interest rate changes at selected maturities. ${ }^{1}$

Our paper is also related to the literature that measures the effects of unconventional monetary policy on the yield curve, and more broadly the literature on the effects of monetary policy announcements using high-frequency identification, such as Kuttner (2001), Gürkaynak, Sack and Swanson (2005a, 2005b, 2007), Wright (2012) and Altavilla and Giannone (2017). While our work builds on these contributions, it substantially differs from them: unlike these papers, which focus only on the effects of monetary policy on yields at specific maturities, we use instead shifts in the whole yield curve to identify unconventional monetary policy shocks; furthermore, we study their effects on exchange rates by measuring the response of exchange rates to the whole shift in the term structure due to the policy itself.

The paper is structured as follows. Sections 2 and 3 describe the data and the empirical approach, respectively. Section 4 presents the empirical results on the effects of monetary policy shocks on exchange rates in conventional times, while Section 5 discusses the results for the unconventional period. Section 6 provides an economic analysis of specific episodes and Section 7 discusses the robustness of the results to the presence of informational effects. Section 8 discusses the channels of transmission of monetary policy shocks to exchange rates as well as the effects of relative term structure movements, while Section 9 investigates which

\footnotetext{
${ }^{1}$ There are several other differences between our work and Gali' (2018). An additional difference is that Gali's (2018) results are unconditional, i.e. independent of which shocks affect agents' expectations of interest rates, whereas we condition on monetary policy announcements.
} 
expectations matter the most in different monetary policy regimes. Section 10 concludes.

\section{The Data}

The term structure data are daily zero-coupon yields (mnemonics "SVENY") from Gürkaynak, Sack and Wright (2007) and include yields at 1 to 30 years maturities. The daily frequency is dictated by the availability of the data. The 3- and 6-month daily zero-coupon yields are from the Federal Reserve Board (Fed) H-15 release. The data are from January 1995 to June 2016. The sample starts in 1995 due to the fact that the Fed did not release statements of monetary policy decisions after its Federal Open Market Committee meetings before 1994. Note that the frequency of the data is daily. While one might be interested in investigating the identification at a higher frequency, Gürkaynak, Sack and Swanson (2007a) show that daily data are sufficient for extracting monetary policy shocks using a high-frequency identification if the sample is limited to post-1995 data, which is our case.

The nominal bilateral exchange rate data for the Euro, British pound, Canadian dollar and the Yen vis-a'-vis the U.S. dollar (respectively denoted by EURUS, GBPUS, CADUS and YENUS) are from Bloomberg. We calculate the daily exchange rate change (measured as foreign currency units for one US dollar) as the (log of the) value at the end of the day minus that at the end of the previous day. ${ }^{2}$ The exchange rate data are in units of foreign currency for one US dollar (USD); thus, in this paper, an increase in the exchange rate denotes an appreciation of the US dollar relative to the foreign currency.

The dates of US conventional monetary policy announcements are from Nakamura and Steinsson (2018) and include Federal Open Market Committee (FOMC) meetings. The unconventional monetary policy announcement dates are instead from Wright (2012), although we updated them to the end of our sample. In particular, the unconventional monetary policy dates include the announcements of the start of LSAP-I on November 25, 2008; LSAP-II on August 10, 2010; and LSAP-III on September 13, 2012; as well as announcements of

\footnotetext{
${ }^{2}$ The EUR/USD series starts on $1 / 1 / 2000$.
} 
additional Treasury and bond purchases, among others. ${ }^{3}$

\section{The Empirical Approach}

Our goal is to measure the effects of monetary policy on exchange rates using a more comprehensive measure of monetary policy shocks. Our measure of monetary policy shocks is an exogenous shift in the entire term structure of interest rates. The idea is that the term structure contains important information on the expected path of future interest rates as well as any changes perceived by financial markets, associated with their perception of riskiness and uncertainty in the economy.

In what follows, we first describe our approach to shock identification; then, we discuss how we estimate the exchange rate responses.

\subsection{Shock Identification}

Let $\mathcal{Y}_{\tau, t}$ denote the yield to maturity at time $t$, where $\tau=\tau_{1}, \tau_{2, \ldots}, \tau_{M}$ is the maturity expressed in years, and $M$ is the number of maturities considered by the researcher. We assume that, on days of a monetary policy announcement, the change in the yield curve is mainly caused by monetary policy actions. The monetary policy shock is thus the change in the term structure on the day of the announcement:

$$
\varepsilon_{t}^{m p}(\tau)=\Delta \mathcal{Y}_{\tau, t} \cdot d_{t},
$$

where $\Delta \mathcal{Y}_{\tau, t} \equiv \mathcal{Y}_{\tau, t}-\mathcal{Y}_{\tau, t-1}$ is the change in the yield curve as a function of maturity $\tau$ on any day $t ; d_{t}$ is a dummy variable equal to unity on a day of a monetary policy announcement, and zero otherwise. Each monetary policy shock can be potentially different: for example, it could manifest itself as a parallel shift in the term structure; or it could affect its slope by affecting more (less) short-term interest rates relative to long-term ones; or it could affect the curvature by affecting the medium-term rates more than the rest of the maturities -

\footnotetext{
${ }^{3}$ See Inoue and Rossi (2017a) and its Not-for-Publication Appendix (Inoue and Rossi, 2017b) for details on the announcement dates.
} 
or, it could be a combination of all these. These different dimensions of monetary policy are embedded in changes in the yield curve associated with monetary policy moves, which we estimate by $\varepsilon_{t}^{m p}(\tau)$ (see Inoue and Rossi, 2017a). Note that the monetary policy shock depends on the maturity, $\tau$.

Figure 1 illustrates the approach. Panel A in the figure plots a specific monetary policy shock in the unconventional period. The solid line in the top graph in Figure 1, Panel A, depicts the term structure before the announcement (made on 16/12/2008) that the Fed Funds Rate reached the zero lower bound while the dotted line with asterisks depicts the term structure right after the announcement. Clearly, the monetary policy event is associated with a downward shift in the whole yield curve, especially at medium-term maturities. The difference between the term structure before and after the announcement is the monetary policy shock that we use in this paper. The shock itself, $\varepsilon_{t}^{m p}(\tau)$, is plotted in the bottom graph in Figure 1, Panel A: clearly the difference between the yield curve before and after the announcement is negative, indicating an expansionary shock, and larger at medium to long maturities, indicating that the shock is mainly perceived in the medium run.

Panel B in Figure 1 plots the shocks for several important monetary policy announcements in the unconventional period, including announcements of LSAP-I, II and III (on 25/11/2008, 10/8/2010 and 13/9/2012, respectively), additional Treasury Purchases (3/11/2010) and the maturity extension program (21/9/2011 and 20/6/2012). As it is clear from the pictures, the monetary policy shocks appear to have different shapes depending on the type of announcement. Our goal in the following sections is exactly to study how the different shapes affect exchange rates' responses.

\section{INSERT FIGURE 1 HERE}

On the one hand, our approach to measuring monetary policy shocks is quite different from that in the traditional exchange rate literature. In the traditional literature, such as Clarida and Gali', 1994, and Eichenbaum and Evans, 1996), the monetary policy shock is identified as the exogenous change in the short-term interest rate (e.g. the Fed Funds rate in the US). By considering changes in the whole term structure, we can comprehensively 
measure the overall stance of an exogenous monetary policy action, whether it is just an unexpected change in the short-term interest rate or the shift in financial market expectations of future interest rates associated to, e.g., quantitative easing (QE) announcements. On the other hand, our "high-frequency" identification approach builds on Kuttner (2001) and Gürkaynak, Sack and Swanson (2005a,b, 2007), although it differs from them as we focus on the change in both the shape and the magnitude of the whole yield curve.

Measuring monetary policy shocks as shifts in the term structure in a short window of time around a monetary policy announcement allows us to identify the exogenous variation in monetary policy under the assumption that any other shocks during the same period of time have only minor effects. This assumption is credible in our context since the window we rely upon is one day. The approach is convenient since it captures only monetary policy changes that are fully unexpected by financial markets. It is important to note that these shocks are monetary policy shocks only if the information set of the private agents coincides with that of the central bank. However, one might worry that the change in expectations may be due to an informational effect, rather than monetary policy, if the Central bank conveys new information about the state of the economy to private agents via its announcements; we verify below that this is not the case in the majority of the events. Also, note that monetary policy shocks that are intended by the policymaker to be expansionary may actually be contractionary if they are not as expansionary as financial markets expect. Similarly to the traditional high frequency identification approach, such shocks will be contractionary in our framework.

We measure the monetary policy shock as the change in the US term structure in a short window of time around the announcement date, as opposed to the change in the US term structure relative to the foreign term structure: under the realistic assumption that the foreign monetary policy authority does not react to a US shock within the same window of time, we capture a genuine US monetary policy shock. On the other hand, the movements in the US term structure may include changes in markets' expectations of future foreign yields; we incorporate this into our notion of a US monetary policy shock. The reason is that we aim at constructing an overall, comprehensive shock measure. Hence, the same US 
monetary policy shock may affect different exchange rates in a different manner. In a later section we consider shocks to term structure differentials.

Finally, the channels through which monetary policy transmits to exchange rates are two: via movements in real interest rates and via changes in inflation expectations. In a later section, we rely on real exchange rates to shed light on the transmission channels.

\subsection{The Estimation of Exchange Rates' Responses}

Let $s_{i, t}$ denote the log of the nominal bilateral exchange rate of country $i$ vis-à-vis the US dollar (USD) at time $t$, that is the units of that country's currency for one US dollar. Thus, an increase in $s_{i, t}$ denotes an appreciation of the US dollar relative to the foreign country. At each point in time, we estimate the response of the rate of growth of the exchange rate $\left(\Delta s_{i, t} \equiv s_{i, t}-s_{i, t-1}\right)$ to the monetary policy shock $\left(\varepsilon_{t}^{m p}(\tau)\right)$ using Inoue and Rossi's $(2017)$ Functional VAR approach as follows.

Recall that the monetary policy shock is proxied by the combination of the changes in the yields at different maturities on the day of the announcement, i.e. the shift in the term structure on monetary policy announcement dates:

$$
\varepsilon_{t}^{m p}(\tau)=\Delta \mathcal{Y}_{\tau, t} \cdot d_{t} \equiv \Delta \mathcal{Y}_{\tau, t}^{*}
$$

Hence, the "functional shock" is constructed in daily data as the difference between the yield curve at the end of the announcement day and the yield curve on the day before the announcement.

Once we have the shocks, we combine the changes in each of the yields on the announcement day, $\Delta \mathcal{Y}_{\tau, t}^{*}$, to obtain the overall effect of the monetary policy event as the linear combination of the changes in the term structure yields using as weights the derivative of the exchange rate with respect to the respective yield. More formally:

$$
\begin{aligned}
& E\left(\Delta s_{i, t+h} \mid\left\{\mathcal{Y}_{\tau, t}+\varepsilon_{t}^{m p}(\tau)\right\}_{\tau=\tau_{1}}^{\tau_{M}}, \mathcal{I}_{t}\right)-E\left(\Delta s_{i, t+h} \mid\left\{\mathcal{Y}_{\tau, t}\right\}_{\tau=\tau_{1}}^{\tau_{M}}, \mathcal{I}_{t}\right) \\
= & \sum_{\tau=\tau_{1}}^{\tau_{M}} E\left(\frac{\partial \Delta s_{i, t+h}}{\partial \mathcal{Y}_{\tau, t}} \mid \mathcal{I}_{t}\right) \varepsilon_{t}^{m p}(\tau) \\
\equiv & \sum_{\tau=\tau_{1}}^{\tau_{M}} \Delta_{\tau, t}
\end{aligned}
$$


where $\mathcal{I}_{t}$ is the information set at time $t,\left\{\mathcal{Y}_{\tau, t}\right\}_{\tau=\tau_{1}}^{\tau_{M}}$ and $\left\{\mathcal{Y}_{\tau, t}+\varepsilon_{t}^{m p}(\tau)\right\}_{\tau=\tau_{1}}^{\tau_{M}}$ denote the $(M \times 1)$ vector of yields before and after the shock, $E\left(\partial \Delta s_{i, t+h} / \partial \mathcal{Y}_{\tau, t} \mid \mathcal{I}_{t}\right)$ is the impulse response coefficients to a shock in the yield curve at maturity $\tau$ after $h$ period, $h=1,2, \ldots, 15$, and is obtained as follows.

First, we estimate a reduced-form VAR model with the change in the log of the exchange rate and the change in each of the $M$ available raw yields at the daily frequency:

$$
X_{t}=\mu+B_{0}+B_{1} X_{t-1}+\cdots+B_{p} X_{t-p}+u_{t}
$$

where $X_{t}=\left[\Delta \mathcal{Y}_{1 / 4, t}, \Delta \mathcal{Y}_{1, t}, \Delta \mathcal{Y}_{5, t}, \Delta \mathcal{Y}_{10, t}, \Delta \mathcal{Y}_{20, t}, \Delta s_{i, t}\right]^{\prime}, E\left(u_{t} u_{t}^{\prime}\right) \equiv \Sigma$, and $p=2$ in our implementation. ${ }^{4}$

Inverting the VAR model yields a reduced-form moving average:

$$
X_{t}=\widetilde{\mu}+u_{t}+\Theta_{1} u_{t-1}+\ldots
$$

While the monetary policy shocks are directly identified outside the VAR by selecting a small window of time around the announcement, we need additional assumptions to identify the VAR transmission mechanism parameters in the Structural VAR. We assume that the yield curve responds to the exchange rate changes only with a lag. Let $A$ denote the Cholesky factor of $\Sigma$, i.e., $A A^{\prime}=\Sigma$. Write $A$ as

$$
A=\left[\begin{array}{cc}
A_{11} & 0 \\
A_{21} & a_{22}
\end{array}\right]
$$

and $\Theta_{h}$ as

$$
\Theta_{h}=\left[\begin{array}{cc}
\Theta_{11, h} & \Theta_{12, h} \\
\Theta_{21, h} & \theta_{22, h}
\end{array}\right]
$$

where $A_{11}$ and $\Theta_{11, h}$ are $(M \times M), \Theta_{12, h}$ is $(1 \times M), A_{21}$ and $\Theta_{21, h}$ are $(1 \times M)$ and $a_{22}$ and $\theta_{22, h}$ are scalars. The Appendix shows that $\left[E\left(\partial \Delta s_{i, t+h} / \partial \mathcal{Y}_{1 / 4, t} \mid \mathcal{I}_{t}\right), \ldots, E\left(\partial \Delta s_{i, t+h} / \partial \mathcal{Y}_{20, t} \mid \mathcal{I}_{t}\right)\right]$ can be written as

$$
\Theta_{21, h}+\Theta_{22, h} A_{21} A_{11}{ }^{-1} .
$$

\footnotetext{
${ }^{4}$ We use the term structure at the following maturities in our estimation: three months, 1, 5, 10 and 20 years. An alternative approach is to use the entire yield curve fitted using a parametric model following Nelson and Siegel (1987) and Diebold and Li (2006) - see Inoue and Rossi (2017) for the alternative parametric approach.
} 
Thus, the overall effect of the monetary policy event $\varepsilon_{t}^{m p} \equiv\left[\varepsilon_{t}^{m p}(1 / 4), \ldots, \varepsilon_{t}^{m p}(20)\right]^{\prime}$ is

$$
\left(\Theta_{21, h}+\Theta_{22, h} A_{21} A_{11}^{-1}\right) \varepsilon_{t}^{m p}
$$

where $h=0,1, \ldots, H$. See the Appendix for our Bayesian procedure to estimate (6). ${ }^{5}$

Our approach of using individual yields has the following advantages: First, it is nonparametric and, therefore, does not require taking a stand on parametric model. Second, although it is quite common to model the yield curve using three factors, there is some empirical evidence of a fourth factor (e.g. Cochrane and Piazzesi, 2009; Dewachter and Lyrio, 2006; and De Pooter, 2007). Our approach is robust to the presence of an additional factor, even if it shows up only in sporadic monetary policy episodes. This is important for our approach since we view the change in the entire yield curve as a shock. Third, because the cross sectional dimension of yield curve data is small, estimating models or factors introduces sampling errors; by using the individual yield data directly, we do not need to take into account the sampling error.

Our choice of variables in the VAR is parsimonious; hence one might worry about misspecification. For example, the VAR does not include inflation or output, differently from e.g. Eichenbaum and Evans (1995). The reason is that the VAR is estimated at the daily frequency, and the broader set of variables considered by Eichenbaum and Evans are predetermined at that frequency and will not respond within the horizon of our responses (which is a few days). Thus, ignoring them will not bias inference. More generally, on the one hand, the high frequency identification (HFI) approach avoids the shock contamination: if one is interested in identifying only the monetary policy shock, under the HFI assumption it will be appropriately identified even if the VAR is misspecified. On the other hand, if the model is misspecified (e.g. because we did not include an important variable), the transmission might be incorrectly estimated. Section 8 includes an analysis where the endogenous movements of the foreign term structure are taken into account, in the sense that the shock is measured as the exogenous movement in the US term structure relative to the foreign term structure.

To allow for changes in the transmission mechanism in different monetary policy periods,

\footnotetext{
${ }^{5}$ The VAR is estimated using Bayesian methods to control for parameter proliferation - see the Appendix for more details.
} 
we estimate eq. (3) in two sub-samples: the conventional monetary policy period (1995:12008:10) and the unconventional period (2008:11-2016:6). Note that the start of the second sub-sample is marked by the start of the first large scale asset purchasing program (LSAP-I), dated November 25, 2008.

In what follows, we separately analyze the effects of conventional and unconventional monetary policy. The next section focuses on monetary policy in conventional times, while the following section focuses on unconventional times. We also decompose the response in each component $\Delta_{\tau, t}$ separately and discuss the results in Section 9 .

\section{Measuring the Effects of Monetary Policy on Ex- change Rates in Conventional Times}

In this section we study the effects of monetary policy shocks on exchange rates in the conventional monetary policy period. By conventional monetary policy we mean situations where the monetary authority's instrument is the short-term interest rate. In our data, the conventional period lasts from the beginning of our sample until the end of October 2008 (included).

Our results are depicted in Figure 2. Each of the figures 2A-D corresponds to a different exchange rate: the US dollar vis-a'-vis the UK pound (depicted in Figure 2A), the Euro (Figure 2B), the Canadian dollar (Figure 2C) and the Yen (Figure 2D). In each figure, we separately consider contractionary and expansionary monetary policy moves as well as their impact at the short- and medium-end of the term structure, depicted in four panels: Panels I and III focus on events traditionally referred to as contractionary monetary policy, as they increase the short-term interest rate, while Panels II and IV focus on expansionary monetary policy.

INSERT FIGURE 2 HERE

In particular, Panel I focuses on fully contractionary monetary policy shocks; that is, 
shocks that are contractionary at both very short- and medium-term maturities, ${ }^{6}$ and where the effect at the medium-end of the term structure is even more contractionary than that on short-term rates (that is, $\Delta \mathcal{Y}_{1 / 4, t}^{*}>0$ and $\Delta \mathcal{Y}_{5, t}^{*}-\Delta \mathcal{Y}_{1 / 4, t}^{*}>0$ ). The graph on the right in Panel I depicts the monetary policy shock as a function of the maturity (in years). Thus, the events depicted in Panel I correspond to monetary policy announcements where the term structure increased at the selected maturities; in fact, the difference between the interest rates after and before the announcement $\left(\varepsilon_{t}^{m p}(\tau)\right)$ is positive. Since the shock is contractionary, agents revise their expectations of current and future interest rates upwards, and even more so for future interest rates.

Panel II, instead, considers fully expansionary shocks, that is shocks that decrease both the short- and the medium-end of the term structure, and are such that the effects are perceived to be even more expansionary in the medium-run than in the short-run (that is, $\Delta Y_{1 / 4, t}^{*}>0$ and $\left.\Delta Y_{5, t}^{*}-\Delta Y_{1 / 4, t}^{*}<0\right)$. We also separately consider cases in which monetary policy is more contractionary at short than at long maturities (Panel III, where $\Delta Y_{1 / 4, t}^{*}<0$ and $\Delta Y_{5, t}^{*}-\Delta Y_{1 / 4, t}^{*}>0$ ), and cases in which monetary policy is less expansionary at long than at short maturities (Panel IV, where $\Delta Y_{1 / 4, t}^{*}>0$ and $\Delta Y_{5, t}^{*}-\Delta Y_{1 / 4, t}^{*}<0$ ). That is, Panel III focuses on cases in which agents expect interest rates to increase in the short-run but not to increase as much (or even decrease) in the long-run. On the contrary, Panel IV considers cases in which the reaction at the short end of the yield curve is expansionary while medium-term yields do not decrease as much as short-term ones (or may even increase).

Each panel has two graphs: as we mentioned, the graph on the right-hand side depicts the monetary policy shocks; on the left hand side, instead, we depict the exchange rate response to each of the shocks depicted on the right hand side. Note that each monetary policy shock is potentially different in both magnitude and shape across maturities, as it can potentially move the yield curve in a different way. Thus, we depict several exchange rate responses, one for each of the monetary policy shocks. Note that the responses are in the same units as the exchange rate (in growth rates).

Our results show that, on average, for all the bilateral exchange rates that we consider, a

\footnotetext{
${ }^{6}$ The very short-term maturity is 3 months and the medium-term maturity is 5 years.
} 
monetary policy tightening (easing) during the conventional monetary policy period generally leads to an appreciation (depreciation) of the US dollar, consistently with the results in Clarida and Gali' (1994), Eichenbaum and Evans (1996) and Faust and Rogers (2003). This result can be appreciated by looking at the two graphs in the top panels in Figures 1A-1D that distinguish between shocks that are fully contractionary and fully expansionary (Panels I and II). For all countries except Canada, a US monetary policy tightening typically results in an appreciation of the US dollar. Similarly, a US monetary easing typically results in a depreciation, as shown in Panel II, where the magnitude again depends on the specific shape of the variation in the yield curve.

Importantly, in most cases the exchange rate response is consistent with Dornbusch's (1976) overshooting model, which predicts that contractionary monetary policy shocks generate a large initial appreciation followed by subsequent depreciations. In fact, at the daily frequency we do not observe the puzzling persistent appreciations typically seen in monthly data studies such as Eichenbaum and Evans (1996). ${ }^{7}$

However, note that the effects of monetary policy depend on how it affects agents' expectations and their perception of risk in the short-versus the medium- and long-run. The exchange rate response, in fact, depends on how the yield curve shifts as a result of monetary policy moves. In the conventional identification approach, shocks of different magnitude result in parallel shifts in the responses, as they only depend on the effect of monetary policy on interest rates at the short-term maturity; in our approach, shocks of different shape may result in exchange rate responses with more complex shapes.

For example, notice how, in the UK pound-US dollar exchange rate, responses with very similar short-run magnitude end up having very different effects on exchange rates. For example, in Panel III in Figure 2A, all shocks increase short-term interest rates; however, their effects on medium- and long-term interest rates are very different, and hence the response of

\footnotetext{
${ }^{7}$ The responses we depict are for the change in the exchange rate. To obtain the responses of the level of the exchange rate one needs to cumulate them. When the response changes sign after the initial impact, the cumulative response will not be hump-shaped and, hence, it is consistent with Dornbusch's (1976) overshooting hypothesis.
} 
exchange rates differs. ${ }^{8}$ This example clearly illustrates the differences between the approach to identification that we use in this paper and the conventional identification: in the conventional Cholesky identification approach, these two shocks would be indistinguishable since they are characterized by a similar increase in the 3-month interest rate, and would thus end up having a similar effect on exchange rates. However, it is clear that they do not have the same effect in our approach. Furthermore, this example clarifies how our approach is different from a VAR where researchers focus on a few interest rates on selected maturities: by selecting only the 3-month and the 5-year maturities, the researcher would be unable to distinguish the two shocks, as they are the same at these maturities - thus leading to incorrect empirical conclusions, as the shocks are very different at other maturities.

Our results point to several differences in the international transmission mechanism of US monetary policy shocks. In fact, note how different the responses of the exchange rate are to the same US monetary policy shock. For example, the effects of a US monetary policy easing are larger in Japan than in any of the other countries.

Panel III in Figures 2A-D focuses on the case where the monetary policy shock is contractionary at short maturities but is perceived not quite as contractionary at medium-term maturities, that is, the 5-year interest rate is expected to be lower than the 3-month one. Such shocks typically lead to a short-run appreciation of the US dollar or, only in the case of Canada, to a short-run depreciation. On the contrary, Panel IV depicts results for the case where the shock is perceived to be expansionary in the short-run but not as much in the medium run; in such cases, the exchange rate may either appreciate or depreciate. Again, one can immediately appreciate how different this result would be in the conventional identification approach, which only focuses on changes in short-term rates.

\section{INSERT FIGURE 3 HERE}

We now turn to discussing in detail the differences between our results and the traditional

\footnotetext{
${ }^{8}$ In particular, unreported results show that the shock that leads to the largest decrease in 2-3 years interest rates ends up causing a depreciation of the dollar, while the opposite is true for the shocks that leads to an increase in the interest rates at similar maturities.
} 
approach. Note that the information in the raw yield curve data at the shortest maturities is described by the 3 -month maturity rate, $\mathcal{Y}_{1 / 4, t}$. Thus, one can replicate the traditional approach typically adopted in the literature (maintaining the high frequency identification) as the special case where the VAR includes only the exchange rate and $\mathcal{Y}_{1 / 4, t}$. In that case, the response of the exchange rate to the monetary policy shock is:

$$
\begin{aligned}
& E\left(\Delta s_{i, t+h} \mid \mathcal{Y}_{1 / 4, t}+\varepsilon_{t}^{t r a d}(1 / 4), \mathcal{I}_{t}\right)-E\left(\Delta s_{i, t+h} \mid \mathcal{Y}_{1 / 4, t}, \mathcal{I}_{t}\right) \\
= & E\left(\frac{\partial \Delta s_{i, t+h}}{\partial \mathcal{Y}_{1 / 4, t}} \mid \mathcal{I}_{t}\right) \varepsilon_{t}^{t r a d}(1 / 4) .
\end{aligned}
$$

Note that the magnitude of the responses in our framework is different from that in the traditional approach, however. In our approach, the magnitude of the response is the actual change in the rate of growth of the exchange rate due to the monetary policy shock, and it is not normalized in standard deviation units. Hence, our responses cannot be directly compared to those in the literature. Furthermore, most of the previous literature estimates VARs with exchange rates in levels rather than in first differences.

Figure 3 revisits the empirical evidence based on the traditional approach, eq. (7). We distinguish between expansionary and contractionary monetary policy, depending on whether the change in the 3 -month rate is positive or negative (i.e. $\Delta Y_{1 / 4, t}>0$ for contractionary traditional shocks and $\Delta Y_{1 / 4, t}<0$ for expansionary ones) . Our results confirm that, even in our review of the traditional approach, contractionary (expansionary) shocks lead to currency's appreciation (depreciation).

Notice however how, in the traditional approach, the responses are proportional to each other: in fact, other dimensions of monetary policy besides changes in the 3-month interest rate are completely ignored and the exchange rate responses are the same up to a scaling factor, the magnitude depending on the change in the (scalar value of the) short-term interest rate. In fact, the reason why only one response is reported in the conventional approach is exactly because the responses are proportional to each other and they only differ by the magnitude of the contemporaneous effect. In particular, notice how an expansionary shock in this case always leads to an exchange rate depreciation, no matter how monetary policy affects expectations in the medium and long-run. In our framework, instead, the reaction of exchange rates is much richer, as it depends on how the term structure changes at different 
maturities. ${ }^{9}$

\section{Measuring the Effects of Monetary Policy on Ex- change Rates in Unconventional Times}

We now turn to analyzing the differences between conventional and unconventional monetary policy. By unconventional monetary policy we mean situations where the Central bank cannot affect the short-term interest rate (as it is stuck at the zero lower bound), and instead either purchases assets to counteract the tightening in financial markets or decrease uncertainty ("Large Scale Asset Purchases", or LSAP in short) or issues announcements about the future path of interest rates that convey information on the length of the zero lower bound period ("Forward guidance"). The start of the unconventional monetary policy period in the US is marked by the first LSAP, in November 2008, although forward guidance was allegedly implemented as a policy instrument since the early 2000 (Gürkaynak, Sack and Swanson, 2005a). Note that our framework does automatically capture both LSAP and forward guidance directly in the way monetary policy shifts in the entire yield curve.

Figure 4 depicts the exchange rate response to the monetary policy shock. Since in the unconventional period short-term interest rates are stuck at the zero-lower bound and cannot be moved further, we distinguish between contractionary and expansionary policy based solely on changes in medium-term interest rates, depicted in Panels I and II respectively. The medium term is defined to be 5 years. That is, expansionary monetary policy shocks are defined as shocks where the change in the 5-year yield on an announcement date is negative $\left(\Delta Y_{5, t}^{*}<0\right)$, while contractionary shocks are such that it is positive $\left(\Delta Y_{5, t}^{*}>0\right)$.

The graphs on the right in Figure 4, Panels I and II, depict the US monetary policy shocks in the unconventional period $\left(\varepsilon_{t}^{m p}(\tau)\right)$. As the figures show, the monetary policy shock is zero at the short-end of the yield curve, and progressively moves away from zero at

\footnotetext{
${ }^{9}$ Note that, even for the traditional monetary policy shock, the empirical evidence is more in line with the overshooting hypothesis (except for Japan) than traditional studies based on monthly or quarterly data. This suggests that the daily frequency in our data may be a crucial element to uncover overshooting.
} 
the long end of the yield curve. This reflects the well-known fact that, in the unconventional period, monetary policy mostly operates by affecting medium- and long-term expectations. Notice, however, how the expected lift-off from the zero lower bound is very different across episodes: in some cases it is more gradual while in others it is more sudden.

\section{INSERT FIGURE 4 HERE}

Comparing conventional and unconventional monetary policy, thus, it is clear that movements in exchange rates during unconventional monetary policy periods are mostly associated with perceived effects of monetary policy in the medium- and long-run.

By comparing Panels I and II in Figure 4, we find that, on average, expansionary policy depreciates the exchange rate while contractionary policy appreciates it. ${ }^{10}$ The exceptions are Canada and the UK, for which expansionary policies may result in both appreciation and depreciations.

By comparing Figures 3 and 4, we draw the following main conclusion: overall, the effects of unconventional monetary policy are similar to those in the conventional period: expansionary monetary policy shocks in the US typically result in a depreciation of the US dollar. The magnitudes are also similar.

Our empirical results are related to Rogers, Scotti and Wright (2014, 2016) and Glick and Leduc (2015), who have investigated the effects of unconventional monetary policy on exchange rates as well. However, there are several important differences between our paper and theirs. A first difference is that, in the latter papers, the shock is the exogenous change in the principal component(s) extracted from a cross section of interest rates, while in our work the shock is the entire shift in the entire term structure due to an exogenous monetary policy move. It is the analysis of how the whole yield curve shifts over time that allows us to crucially differentiate our results from theirs. A second, important difference is that Rogers, Scotti and Wright (2014) and Glick and Leduc (2015) use an event study approach which allows them to estimate the contemporaneous correlation between changes in the term

\footnotetext{
${ }^{10}$ Our unconventional sample includes some episodes of contractionary policy.
} 
structure due to monetary policy on specific dates and the exchange rate, but is otherwise silent on the dynamic effects; in contrast, our paper estimates the whole dynamic impulse response. Rogers, Scotti and Wright $(2014,2016)$ also complement their analyses with VARs either using a heteroskedasticity-based identification (as in Rogers, Scotti and Wright, 2014, and Wright, 2012), or external instruments (as in Rogers, Scotti and Wright, 2016) to trace

out the effects of monetary policy shocks over time. Our approach instead relies on the Functional VAR approach (Inoue and Rossi, 2017a), which provides the dynamic response to the shift in the whole term structure viewed as a function of maturity. Finally, our analysis naturally leads to time-varying responses of exchange rates that fundamentally depend on the ways in which monetary policy affects agents' expectations of current and future interest rates as well as the risk and uncertainty in the economy.

\section{How Do Exchange Rates Respond to Monetary Pol- icy Shocks?}

In order to understand how exchange rates move after a monetary policy announcement, let's focus on the specific events during the unconventional monetary policy period depicted in Figure 1. Figure 5 plots the response of exchange rates to each of these shocks.

Recall from Figure 1 that large scale asset purchases typically decrease yields at most maturities. The decrease has a clear hump-shaped pattern, with the largest decrease showing up on yields at the medium-term (5 years) maturity; interestingly, the effects of LSAP are less important in the longer run as we move from LSAP-I to LSAP-III, and in the latter case yields at longer maturities increase. LSAPs typically result in a depreciation of the US dollar. In particular, Figure 5 shows that LSAP-I appreciates the US dollar against the UK pound and the Canadian dollar, and depreciates against the euro and the yen, while LSAP II and III result in an immediate depreciation in the exchange rate relative to all countries. The treasury security purchases announcement on 12/1/2008 decrease the yield curve as well, and the effects are larger at longer maturities than at shorter ones. Again, as a result, the exchange rate depreciates relative to all countries. 
The responses contrast sharply with those associated with the maturity extension announcements on $9 / 21 / 2011$ and $6 / 20 / 2012$. The latter increase the yield curve at short maturities and decrease it a long maturities, and result in a broad appreciation of the US dollar against all currencies.

\section{INSERT FIGURE 5 HERE}

\section{The Information Channel}

The identification approach in the previous sections requires that monetary policy announcements carry information about monetary policy changes, as opposed to new information about the state of the economy. The idea that monetary policy announcements affect agents' beliefs about economic fundamentals, and not only about monetary policy, has been proposed by Nakamura and Steinsson (2018), and is referred to as "the information channel". Whether the information channel is empirically relevant is an open question that has attracted a lot of interest. The information channel is more plausible if Central banks have superior information about the state of the economy relative to market participants. On the one hand, Romer and Romer (2000) and Nakamura and Steinsson (2018) found evidence that this is the case in the US on average over a long sample of data. On the other hand, Rossi and Sekhposyan $(2016,2018)$ investigate how the Fed's superior information content has evolved over time and show that, in the last decade, the Central bank lost its informational advantage; hence, the latter suggest that the informational channel may not be too important in our empirical analysis.

To verify the robustness of our results to the presence of information channel effects, we construct an informationally-robust measure of monetary policy shocks along the lines of Miranda-Agrippino and Ricco (2018). The presence of an informational channel implies that a monetary policy shock identified in a VAR will be the convolution of the true monetary policy shock and an endogenous response to the information contained in the announcement about the future state of the economy. Such information should be contained in the Central 
bank's own forecast of the future state of the economy or its own revisions of previous forecasts. As the proxy for the Central bank forecast we use the Greenbook forecasts of inflation, output and unemployment. The informationally-robust measure of monetary policy shocks is therefore constructed as the residual from a regression of our functional monetary policy shock on the Greenbook forecasts:

$$
\varepsilon_{t}^{m p}(\tau)=\gamma_{1}^{\prime} F_{t}^{c b} x_{q-1}+\gamma_{2}^{\prime}\left(F_{t}^{c b} x_{q-1}-F_{t-1}^{c b} x_{q-1}\right)+\varepsilon_{t}^{m p i}(\tau)
$$

where $\varepsilon_{t}^{m p i}(\tau)$ is the informationally-robust monetary policy shock; $F_{t}^{c b} x_{q-1}$ denotes the Greenbook forecasts of inflation, real output growth and unemployment made at time $t$ for the previous quarter, and $\left(F_{t}^{c b} x_{q-1}-F_{t-1}^{c b} x_{q-1}\right)$ denotes the forecast revision. ${ }^{11}$

\section{INSERT FIGURE 6 HERE}

Figure 6 repeats the analysis in Figure 2 using the monetary policy shock robust to informational effects, $\varepsilon_{t}^{m p i}(\tau) .{ }^{12}$ By comparing Figure 6 with Figure 2, we note that our broad empirical findings are qualitatively unchanged: fully contractionary/expansionary shocks lead to an exchange rate appreciation/depreciation (Panels I and II in Figure 6). The other shocks depicted in panels III and IV in Figure 6 lead, as before, to heterogeneous responses; in some cases, the average response changes after taking into account informational effects (as in the case of Panels III for the UK and Europe).

\footnotetext{
${ }^{11}$ Given the small sample we have available, we can include only a limited number of regressors. Thus, we include only the forecasts for the previous quarter as opposed to the nowcast and forecasts for future values, as the latter are less significant overall when included in regression (8) across countries. In other words, we estimate exactly equation (8).

${ }^{12}$ Note that the analysis focuses on the conventional period since, in the unconventional period, regression (8) would estimate a non-zero adjusted short-term interest rate even if the short-term interest rate is at the zero lower bound. Also, estimating regression (8) in the zero lower bound period is impractical as the number of observations is very small.
} 


\section{The Transmission of Monetary Policy Shocks}

In this section, we ask two questions. The first is whether the transmission of monetary policy shock involves changes in real interest rates or changes in inflation expectations. The second is what are the effects of movements in the relative term structures on exchange rates. We restrict our sample to the UK and Europe and the unconventional period, due to data limitations.

\subsection{Expected Real Interest Rates Versus Inflation Expectations}

Consider the traditional UIRP condition, which states that: ${ }^{13}$

$$
i_{t}^{f}=i_{t}+E_{t}\left(s_{t+1}-s_{t}\right)
$$

where $s_{t}$ is the log of the nominal exchange rate (that is, the units of foreign currency for one US dollar), $i_{t}$ is the nominal interest rate in the domestic country and $i_{t}^{f}$ is the foreign interest rate. Solving for the exchange rate:

$$
s_{t}=\sum_{\tau=0}^{\infty} E_{t}\left(i_{t+\tau}-i_{t+\tau}^{f}\right)+\lim _{T \rightarrow \infty} E_{t}\left(s_{t+T}\right)
$$

Thus, since one has available expected interest rates $\mathcal{Y}_{\tau, t}=E_{t}\left(i_{t+\tau}\right)$ and $\mathcal{Y}_{\tau, t}^{f}=E_{t}\left(i_{t+\tau}^{f}\right)$ from term structure data, one could substitute them in eq. (10):

$$
s_{t}=\sum_{\tau=0}^{\infty}\left(\mathcal{Y}_{\tau, t}-\mathcal{Y}_{\tau, t}^{f}\right)+\lim _{T \rightarrow \infty} E_{t}\left(s_{t+T}\right),
$$

Hence, the net present value model in eq. (11) suggests that exogenous movements in the term structure of expected nominal interest rates should be reflected in changes in nominal exchange rates. However, changes in the nominal interest rate might be due to either changes in future expected real interest rates or changes in future expected inflation. To see this, let the log of the real exchange rate $q_{t}$ be defined as $q_{t}=p_{t}+s_{t}-p_{t}^{f}$, where $p_{t}$ and $p_{t}^{f}$ are the $\log$ of the home and foreign price levels, respectively. Thus, substituting the latter into eq.

\footnotetext{
${ }^{13}$ We assume that there are no risk premia for the sake of motivating this discussion.
} 
(10), we obtain:

$$
s_{t}=\sum_{\tau=0}^{\infty} E_{t}\left(i_{t+\tau}-i_{t+\tau}^{f}\right)+\lim _{T \rightarrow \infty} E_{t}\left(q_{t+T}+p_{t+T}^{f}-p_{t+T}\right)
$$

Note that $\lim _{T \rightarrow \infty} E_{t}\left(q_{t+T}\right)$ is independent of monetary policy shocks (in deviation from the steady state), while $\lim _{T \rightarrow \infty} E_{t}\left(p_{t+T}^{f}-p_{t+T}\right)$ does depend on monetary policy in the long run, as monetary policy influences expectations of future inflation. A purely expansionary monetary policy shock, for example, might cause nominal interest rates to go down at the short end of the yield curve but up at the long end. Nominal interest rates go down at the short end because expansionary monetary policy lowers real interest rates. But if expansionary monetary policy also increases inflation expectations in the future, that effect may dominate at the longer end of the yield curve, and nominal interest rates may go up for longer maturities. Thus, it is unclear whether monetary policy actions are transmitted to exchange rates via changes in real interest rates or via changes in inflation expectations.

In this section, we investigate whether the transmission channel is indeed via changes in real interest rates. Note that, in a real version of eq. (12), the long-run term on the right-end side of eq. (12) is independent of long-run inflation expectations. ${ }^{14}$ To see this, let $E_{t} \pi_{t+\tau}$ and $E_{t} \pi_{t+\tau}^{f}$ be the expected inflation in the home and foreign countries and let the real interest rate $r_{t}$ be defined as $r_{t} \equiv i_{t}-E_{t}\left(\pi_{t+1}\right)$ - and similarly for the foreign real interest rate, $r_{t}^{f} \equiv i_{t}^{f}-E_{t}\left(\pi_{t+1}^{f}\right)$. Then, the UIRP in eq. (9) can be rewritten as $r_{t}^{f}=r_{t}+E_{t}\left(q_{t+1}-q_{t}\right)$. Hence,

$$
q_{t}=\sum_{\tau=0}^{\infty} E_{t}\left(r_{t+\tau}-r_{t+\tau}^{f}\right)+\lim _{T \rightarrow \infty} E_{t}\left(q_{t+T}\right),
$$

where $E_{t}\left(r_{t+\tau}-r_{t+\tau}^{f}\right)$ can be directly measured from term structure data as the difference between the expected real interest rates at maturity $\tau$ in the domestic and foreign countries, $\mathcal{Y}_{\tau, t}^{r}=\mathcal{Y}_{\tau, t}-E_{t}\left(\pi_{t+\tau}\right)$ and $\mathcal{Y}_{\tau, t}^{r f}=\mathcal{Y}_{\tau, t}-E_{t}\left(\pi_{t+\tau}^{f}\right)$. Since $\lim _{T \rightarrow \infty} E_{t}\left(q_{t+T}\right)$ is independent of monetary policy, conditional on monetary policy shock days we should observe that movements in the real exchange rate reflect movements in the real interest rate. Hence, eq. (13) helps interpreting the results.

\footnotetext{
${ }^{14}$ We are grateful to Jordi Gali' for the precious suggestions regarding the analysis in this section.
} 
Thus, in this section, we redo the same analysis as in the previous section, except that we use real exchange rates and real interest rates instead of their nominal counterparts. Thus, the shock is $\Delta \mathcal{Y}_{\tau, t}^{r *}=\Delta \mathcal{Y}_{\tau, t}^{r} \cdot d_{t}$. Comparing the results based on real versus nominal variables will help us understand whether the effects were driven by movements in expected future real interest rates or in inflation expectations.

We estimate the real interest rate as the nominal interest rate minus the expected inflation: $E_{t}\left(r_{t+\tau}\right)=E_{t}\left(i_{t+\tau}\right)-E_{t}\left(\pi_{t+\tau+1}\right)$. We follow Gali' (2017) in constructing expected inflation from swaps daily zero coupon inflation data. In particular, we use the US inflation linked swap rates from Thomson Reuters Datastream (the mnemonic is USDISxY, where " $\mathrm{x}$ " is the maturity in years).

The real exchange rate is obtained from the nominal bilateral exchange rate by adjusting for the ratio of the price levels of the two countries. The price levels are the CPI indices, monthly and seasonally adjusted. We interpolate the monthly series to obtain daily series assuming a constant growth of the price level over the month. ${ }^{15}$ The price level is measured by the CPI index. The CPI index for the UK is from the British Office for National Statistics, CPI INDEX 00: ALL ITEMS 2015=100, series ID D7BT, available at https://www.ons.gov.uk/economy/ inflationandpriceindices/timeseries/d7bt/mm23. The CPI index for the US is the Consumer Price Index for All Urban Consumers: All Items, Index 1982-1984=100, Monthly, Seasonally Adjusted (CPIAUCSL) from the St. Louis FRED database. For Europe, we use the Euro area (changing composition) HICP Overall index, Working day and seasonally adjusted available at the SDW dataset of the European Central Bank (http://sdw.ecb.europa.eu/ (mnemonics ICP.M.U2.Y.000000.3.INX).

We restrict the analysis to maturities of $1,5,10,15$ and 20 years because of the availability

\footnotetext{
${ }^{15}$ The real exchange rate level $Q_{t}$ is measured as the foreign cost of 1 US dollar times the price in the US and divided by the price in the foreign country and is calculated as follows. Let $m$ and $d$ denote the month and the day that correspond to $t$. Then we assume that $Q_{t}=\left(P_{m-1} / P_{m-1}^{*}\right) S_{t}\left(\left(P_{m} / P_{m}^{*}\right) /\left(P_{m-1} / P_{m-1}^{*}\right)\right)^{(d / 30)}$, where $S_{t}$ is the exchange rate in levels (units of foreign currency for 1 US dollar), $P_{m}$ is the price level in the US in month $m$ and $P_{m}^{*}$ is the foreign price level. Since the exchange rate is ultimately used in the estimation in growth rates (that is, we use $\Delta q_{t}=q_{t}-q_{t-1}$ in the VAR, where $q_{t}=\ln Q_{t}$ ), the base year of the price indices does not matter.
} 
of the term structure data (data are unavailable for maturities shorter than one year). The analysis focuses only on the unconventional period since the daily swap data are available starting in mid-2007 for the US and the UK and mid-2008 for Europe. Also, the data are available only for the US, UK and Europe.

Figure 7 shows that, in the unconventional period and for both currencies, a real, expansionary US monetary policy shock depreciates the US dollar and a contractionary one appreciates it, even at the long end of the yield curve. The magnitudes are similar to those in Section 5. Hence, this rules out that the transmission mechanism only operates via expected inflation, and suggests that expected long-run changes in real interest rates triggered by monetary policy play an important role.

\section{INSERT FIGURE 7 HERE}

\subsection{The Response to Changes in Term Structure Differentials on Monetary Policy Announcement Dates}

The main focus of our paper is to quantify the effects of a US monetary policy shock on exchange rates. However, as noted before, a US monetary policy shock may change financial markets' expectations about future foreign interest rates, not just domestic rates. ${ }^{16}$ In this section, we instead investigate the response of exchange rates to the component in the US monetary policy shock that only affects relative interest rate expectations - that is, in our context, relative shifts of domestic versus foreign real yield curves. Note that this is not the response of exchange rates to the domestic monetary policy shock - rather, the response of exchange rates to the movements in the changes in the relative term structure due to the domestic monetary policy shock. Hence, in this section, the shock, $\varepsilon_{t}^{m p}(\tau)$, is defined as the change in the yield curve differential on the day of the monetary policy announcement:

\footnotetext{
${ }^{16}$ In the previous sections, we included the reaction of both domestic and foreign rates in our monetary policy shock notion to obtain a comprehensive measure of the overall US monetary policy shock, no matter whether it affects domestic or foreign markets.
} 
$\Delta \tilde{\mathcal{Y}}_{\tau, t}^{r *}=\Delta \tilde{\mathcal{Y}}_{\tau, t}^{r} \cdot d_{t}$, where $\Delta \tilde{\mathcal{Y}}_{\tau, t}^{r}=\Delta Y_{\tau, t}-E_{t} \pi_{t+\tau}-\left(\Delta \mathcal{Y}_{\tau, t}^{f}-E_{t} \pi_{t+\tau}^{f}\right) \cdot{ }^{17}$

We estimate the response of exchange rates as follows:

$$
\begin{aligned}
& E\left(\Delta s_{i, t+h} \mid\left\{\widetilde{\mathcal{Y}}_{\tau, t}^{r}+\varepsilon_{t}^{m p d i f f}(\tau)\right\}_{\tau=\tau_{1}}^{\tau_{M}}, \mathcal{I}_{t}\right)-E\left(\Delta s_{i, t+h} \mid\left\{\widetilde{\mathcal{Y}}_{\tau, t}^{r}\right\}_{\tau=\tau_{1}}^{\tau_{M}}, \mathcal{I}_{t}\right) \\
= & \sum_{\tau=\tau_{1}}^{\tau_{M}} E\left(\frac{\partial \Delta s_{i, t+h}}{\partial \widetilde{\mathcal{Y}}_{\tau, t}^{r}} \mid \mathcal{I}_{t}\right) \varepsilon_{t}^{m p}(\tau)
\end{aligned}
$$

where $\varepsilon_{t}^{m p}(\tau)=\Delta \widetilde{\mathcal{Y}}_{\tau, t}^{r} \cdot d_{t} \equiv \Delta \widetilde{\mathcal{Y}}_{\tau, t}^{r *}$ and $E\left(\frac{\partial \Delta s_{i, t+h}}{\partial \widetilde{\mathcal{Y}}_{\tau, t}^{r}} \mid \mathcal{I}_{t}\right)$ are estimated from the VAR in eq. (3), where $X_{t}=\left(\Delta s_{i, t}, \Delta \tilde{\mathcal{Y}}_{1 / 4, t}^{r}, \Delta \tilde{\mathcal{Y}}_{1, t}^{r}, \Delta \tilde{\mathcal{Y}}_{5, t}^{r}, \Delta \tilde{\mathcal{Y}}_{10, t}^{r}, \Delta \tilde{\mathcal{Y}}_{20, t}^{r}\right)^{\prime}$, and $h=1,2, \ldots, 15$ is the horizon of the response. ${ }^{18}$ Again, the VAR coefficients are estimated separately in the conventional and unconventional regimes to allow for changes in the transmission mechanism.

Data for zero-coupon yield curves data are from the Bank of England (https://www.bankof england.co.uk/statistics/yield-curves), the European Central Bank Data Warehouse (http:// sdw.ecb.europa.eu/ browse.do?node=9691126) and from the Bank of Canada (https://www. bankofcanada.ca/rates/interest-rates/bond-yield-curves/). Expected inflation in Europe and the UK is constructed from the Euro and UK inflation linked swap zero-coupon rates available at Thomson Reuters Datastream (the mnemonics are GBPISxY and EURISxY, where " $\mathrm{x}$ " is the maturity in years).

Figure 8 depicts the responses. Again, for both currencies, a real, expansionary US monetary policy shock depreciates the US dollar and a contractionary one appreciates it. Hence, the results are similar when considering relative term structure changes.

\section{INSERT 8 HERE}

\section{Which Expectations Matter the Most?}

Given that, in our framework, the monetary policy shock has multiple dimensions, it is important to examine which changes in agents' expectations about future interest rates and

\footnotetext{
${ }^{17}$ As before, $\tau$ is the maturity of the yield in the term structure and $d_{t}$ is a dummy variable indicating days of US monetary policy announcements.

${ }^{18}$ Again, the maturity of the yields is expressed in years.
} 
risk premia cause the exchange rate appreciation/depreciation. We do so by reporting the components of the responses defined in eq. (2). The results are shown in Figures 9 and 10 for the conventional and unconventional periods, respectively. Overall, we find interesting differences across currencies as well as specific episodes, although the results are broadly similar for conventional and unconventional monetary policy regimes.

\section{INSERT FIGURE 9 HERE}

By comparing the shape of the responses, depicted in the graph in the top left corner for each country, with the various components in the decomposition, depicted in the remaining graphs, we draw the following conclusions. In the conventional period, the most important components are the short-term rates (typically one year). The importance of specific maturities depends on the currency: for example, the most important components are the 5 years for the UK, the one year for Europe and Japan, and the 3 months and the 1 year for Canada.

\section{INSERT FIGURE 10 HERE}

In the unconventional period, the exchange rates fluctuations are also driven by longer term maturities, typically 5 years (but also the 10 or 20 years, depending on the country), in addition to the one year maturity. Interestingly, however, fluctuations at the very longend of the yield curve are most important in contractionary episodes while slightly shorter maturities are most important in expansionary episodes. Again, the details depend on the specific country.

\section{Conclusions}

In this paper we investigated the effects of monetary policy shocks on exchange rates. We identify monetary policy shocks as shifts in the whole yield curve in order to analyze how 
changes in agents' expectations of interest rates and changes in risk premia across all maturities dynamically affect exchange rates.

We find that, on average across episodes, the effects of monetary policy shocks on exchange rates are qualitatively similar in both conventional and unconventional periods; in particular, a US monetary policy easing results in a depreciation of the US dollar exchange rate. However, the exchange rate response differs depending on the effects of monetary policy on people's expectations of the interest rate path and risk premia in the short, medium and long run in specific episodes. The monetary policy transmission mechanism does involve changes in real interest rates, even at long maturities. Thus, our approach can help in quantifying and further advancing our understanding of the different dimensions of monetary policy first discussed in Gürkaynak, Sack and Swanson (2005a). Furthermore, we find empirical evidence in favor of the overshooting hypothesis in our daily data. 


\section{References}

Altavilla, Carlo and Domenico Giannone (2017), "The Effectiveness of Non-Standard Monetary Policy Measures: Evidence from Survey Data", Journal of Applied Econometrics $32(5), 952-964$.

Bouakez, Hafedh and Michel Normandin (2010), "Fluctuations in the Foreign Exchange Market: How Important Are Monetary Policy Shocks?", Journal of International Economics.

Clarida, Richard and Jordi Gali' (1994). "Sources of Real Exchange Rate Fluctuations: How Important Are Nominal Shocks?", Carnegie Rochester Conference Series on Public Policy 41, 1-56.

Cochrane, J.H., and M. Piazzesi (2009), "Decomposing the Yield Curve," mimeo, University of Chicago.

De Pooter, M. (2007), "Examining the Nelson-Siegel Class of Term Structure Models," mimeo, Tinbergen Institute.

Dewachter, H., and M. Lyrio (2006), "Macro Factors and the Term Structure of Interest Rates," Journal of Money, Credit and Banking 38, 119-140.

Diebold, Francis X. and Canlin Li (2006), "Forecasting the Term Structure of Government Bond Yields," Journal of Econometrics 130(2), 337-364.

Dornbusch, Rudiger (1976), "Expectations and Exchange Rate Dynamics", Journal of Political Economy 84, 1161-76.

Eichenbaum, Martin and Charles Evans (1996), "Some Empirical Evidence on the Effects of Monetary Policy Shocks on Exchange Rates," Quarterly Journal of Economics 1-22.

Faust, Jon and John Rogers (2003), "Monetary Policy's Role in Exchange Rate Behavior," Journal of Monetary Economics 1-54.

Gali', Jordi (2018), "Forward Guidance and the Exchange Rate," Barcelona GSE Working Paper n. 1021. 
Glick, Reuven and Sylvain Leduc (2015), "Unconventional Monetary Policy and the Dollar: Conventional Signs, Unconventional Magnitudes", Federal Reserve Bank of San Francisco W.P. 2015-18.

Gürkaynak, Refet S., Bryan Sack and Eric Swanson (2005a), "Do Actions Speak Louder Than Words? The Response of Asset Prices to Monetary Policy Actions and Statements", International Journal of Central Banking 1(1),55-93.

Gürkaynak, Refet S., Bryan Sack and Eric Swanson (2005b), "The Sensitivity of LongTerm Interest Rates to Economic News: Evidence and Implications for Macroeconomic Models," American Economic Review 95(1), 425-436.

Gürkaynak, Refet S., Bryan Sack and Eric Swanson (2007), "Market-Based Measures of Monetary Policy Expectations," Journal of Business and Economic Statistics 25, 201-212.

Gürkaynak, Refet S., Bryan Sack and Jonathan Wright (2007), "The U.S. Treasury Yield Curve: 1961 to the Present", Journal of Monetary Economics 54(8), 2291-2304.

Kuttner, Kenneth (2001), "Monetary Policy Surprises and Interest Rates: Evidence from the Federal Funds Futures Market," Journal of Monetary Economics 47 (3), 523-544.

Inoue, Atsushi and Barbara Rossi (2017), "The Effects of Conventional and Unconventional Monetary Policy: A New Approach", mimeo.

Inoue, Atsushi and Barbara Rossi (2017b), "Not-for-Publication Appendix to: The Effects of Conventional and Unconventional Monetary Policy: A New Approach", mimeo.

Miranda-Agrippino, Silvia and Giovanni Ricco (2018), "The Transmission of Monetary Policy Shocks", Bank of England Working Paper.

Nakamura, Emi and Jon Steinsson (2018), "High Frequency Identification of Monetary Non-Neutrality: The Information Effect," Quarterly Journal of Economics 133(3), 12831330. 
Neely, Christopher J. (2015), "Unconventional Monetary Policy Had Large International Effects," Journal of Banking \&3 Finance 52(C), 101-111.

Nelson, Charles R. and Andrew F. Siegel (1987), "Parsimonious Modeling of Yield Curve", Journal of Business 60, 473-489.

Rogers, Jon, Chiara Scotti and Jonathan H. Wright (2014), "Evaluating Asset-Market Effects of Unconventional Monetary Policy: A Multi-Country Review," Economic Policy 29, $3-50$.

Rogers, Jon, Chiara Scotti and Jonathan H. Wright (2016), "Unconventional Monetary Policy and International Risk Premia", Board of Governors IFD Papers No. 1172.

Romer Christina D. and David H, Romer (2000), "Federal Reserve Information and the Behavior of Interest Rates", American Economic Review 90(3), 429-457.

Rossi, Barbara and Tatevik Sekhposyan (2016), "Forecast Rationality Tests in the Presence of Instabilities, With Applications to Federal Reserve and Survey Forecasts", Journal of Applied Econometrics 31(3), 507-532.

Rossi, Barbara and Tatevik Sekhposyan (2018), "Is the Information Channel Empirically Relevant?", mimeo.

Scholl, Almuth and Harald Uhlig (2008), "New Evidence on the Puzzles: Results from Agnostic Identification on Monetary Policy and Exchange Rates," Journal of International Economics 76(1), 1-13.

Swanson, E. (2017), "Measuring the Effects of Federal Reserve Forward Guidance and Asset Purchases on Financial Markets", UC Irvine, mimeo.

Uhlig, Harald (2005), "What Are the Effects of Monetary Policy on Output? Results From an Agnostic Identification Procedure," Journal of Monetary Economics, 52, 381-419.

Wright, Jonathan H. (2012), "What does Monetary Policy do to Long-term Interest Rates at the Zero Lower Bound?," Economic Journal 122, F447-F466. 


\section{Appendix. Details About the Estimation Procedure}

Let $s_{t+h}, y_{t}$ and $I_{t}$ denote an exchange rate at time $t+h$, an $(M \times 1)$ vector of yields at time $t$ and the information set at time $t$ excluding $y_{t}$, respectively. Following Inoue and Rossi (2017a), define

$$
f_{t}\left(y_{t}\right)=E\left(s_{t+h} \mid y_{t}, I_{t}\right)
$$

To simplify the notation, we drop the subscript $t$ from this point on. Then the $h$-step-ahead impulse response of an exchange rate to a yield curve shock $\varepsilon$, where $\varepsilon$ is an $(M \times 1)$ vector of yield shocks, is defined as

$$
\lim _{\alpha \rightarrow 0} \frac{f(y+\alpha \varepsilon)-f(y)}{\alpha}
$$

provided the limit exists.

To make this concept operational, we consider a VAR model of $M$ yields $y_{t}$ and exchange rate returns $s_{t}$ with normally distributed disturbance terms:

$$
X_{t}=B_{0}+B_{1} X_{t-1}+\cdots+B_{p} X_{t-p}+u_{t}
$$

where the last element of $X_{t}$ is the exchange return, $u_{t} \stackrel{i i d}{\sim} N(0, \Sigma)$ and $\Sigma$ is an $(M+1) \times$ $(M+1)$ positive definite matrix. If we are to identify all structural impulse responses, we need to impose the short-run restriction that the yield curve does not contemporaneously respond to exchange rate shocks. In other words, the impact matrix takes the form of:

$$
\left[\begin{array}{ccccc}
x & x & \ldots & x & 0 \\
x & x & \ldots & x & 0 \\
\ldots & \ldots & \ldots & \ldots & \ldots \\
x & x & \ldots & x & 0 \\
x & x & \ldots & x & x
\end{array}\right],
$$

where $x$ denotes a non-zero element. Instead of assuming lower-triangularity, we assume a zero-block restriction; this is because we do not want to assume the recursive ordering among the $M$ yields. To impose the restriction in eq. (19), let

$$
A=\left[\begin{array}{cc}
A_{11} & 0 \\
A_{21} & a_{22}
\end{array}\right]
$$


denote the Cholesky factor of $\Sigma$ such that $A A^{\prime}=\Sigma$, where $A_{11}$ is $(M \times M), A_{21}$ is $(1 \times M)$ and $a_{22}$ is $(1 \times 1)$. Let $Q$ denote a draw from the Haar distribution over the space of $(M \times M)$ orthogonal matrices, such that $Q^{\prime} Q=Q Q^{\prime}=I$, and define

$$
\tilde{A}=A\left[\begin{array}{ll}
Q & 0 \\
0 & 1
\end{array}\right]=\left[\begin{array}{cc}
A_{11} Q & 0 \\
A_{21} Q & a_{22}
\end{array}\right] \text {. }
$$

By construction, $\tilde{A}$ takes the form of (19) and satisfies $\tilde{A} \tilde{A}^{\prime}=\Sigma$. Thus the $h$-step-ahead structural impulse response matrix is given by $\Theta_{h} \tilde{A}$ where $\Theta_{h}$ is the $h$-step-ahead reducedform moving average coefficient matrix. Note that some elements of these structural impulse response matrices are not point-identified but are set-identified, since we allow for arbitrary correlations among the elements in the yield curve shock.

To derive the $h$-step-ahead impulse response of the exchange rate to a yield curve shock $\varepsilon,(17)$, write $\Theta_{h}$ as

$$
\Theta_{h}=\left[\begin{array}{cc}
\Theta_{11, h} & \Theta_{12, h} \\
\Theta_{21, h} & \Theta_{22, h}
\end{array}\right] .
$$

Then the impulse response can be written as

$$
\left(\Theta_{21, h} A_{11} Q+\Theta_{22, h} A_{21} Q\right)\left(A_{11} Q\right)^{-1} \varepsilon=\left(\Theta_{21, h}+\Theta_{22, h} A_{21} A_{11}^{-1}\right) \varepsilon
$$

To estimate the VAR parameters $B_{0}, B_{1}, \ldots, B_{1}$ and $\Sigma$, we use the normal-Wishart family with the uninformative prior parameters for (see Appendix B of Uhlig, 2005, for example) and proceed as follows:

Step 1: Draw $B_{0}, B_{1}, \ldots, B_{p}$ and $\Sigma$ from their posterior distribution and $Q$ from the Haar distribution. Let $A$ denote the Cholesky factor of $\Sigma$.

Step 2: Calculate the $h$-step-ahead reduced-form moving average matrix $\Theta_{h}$ from $B_{1}, \ldots, B_{p}$. That is, $\Theta_{h}$ is the upper-left $(M+1) \times(M+1)$ sub-matrix of the $h$-th power of the companion matrix:

$$
\left[\begin{array}{cccc}
B_{1} & B_{2} & \cdots & B_{p} \\
I_{(M+1)(p-1)} & 0
\end{array}\right]
$$

where $I_{(M+1)(p-1)}$ is the $(M+1)(p-1) \times(M+1)(p-1)$ identity matrix and the 0 is the $(M+1)(p-1) \times(M+1)$ matrix of zeros. A draw of the $h$-step-ahead structural impulse response matrix is $\Theta_{h} \tilde{A}$ where $\tilde{A}=A Q$. 
Step 3: A draw of the $h$-step-ahead structural impulse response of the exchange rate to the yield curve shock $\varepsilon$ is given by (23).

Step 4: Repeat Steps 1-3 many times. 


\section{Figures}

Figure 1, Panel A. US Monetary Policy Shocks
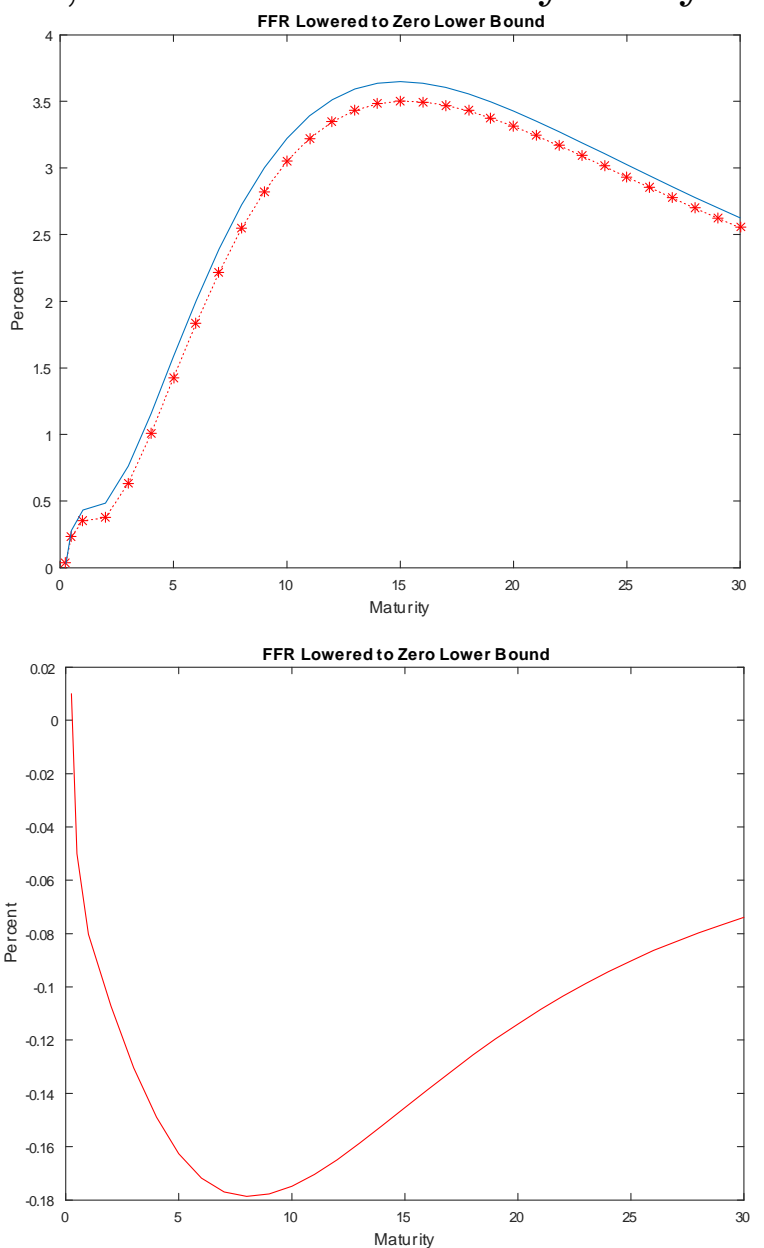
Figure 1, Panel B. US Monetary Policy Shocks

LSAP-I, Nov. 252008
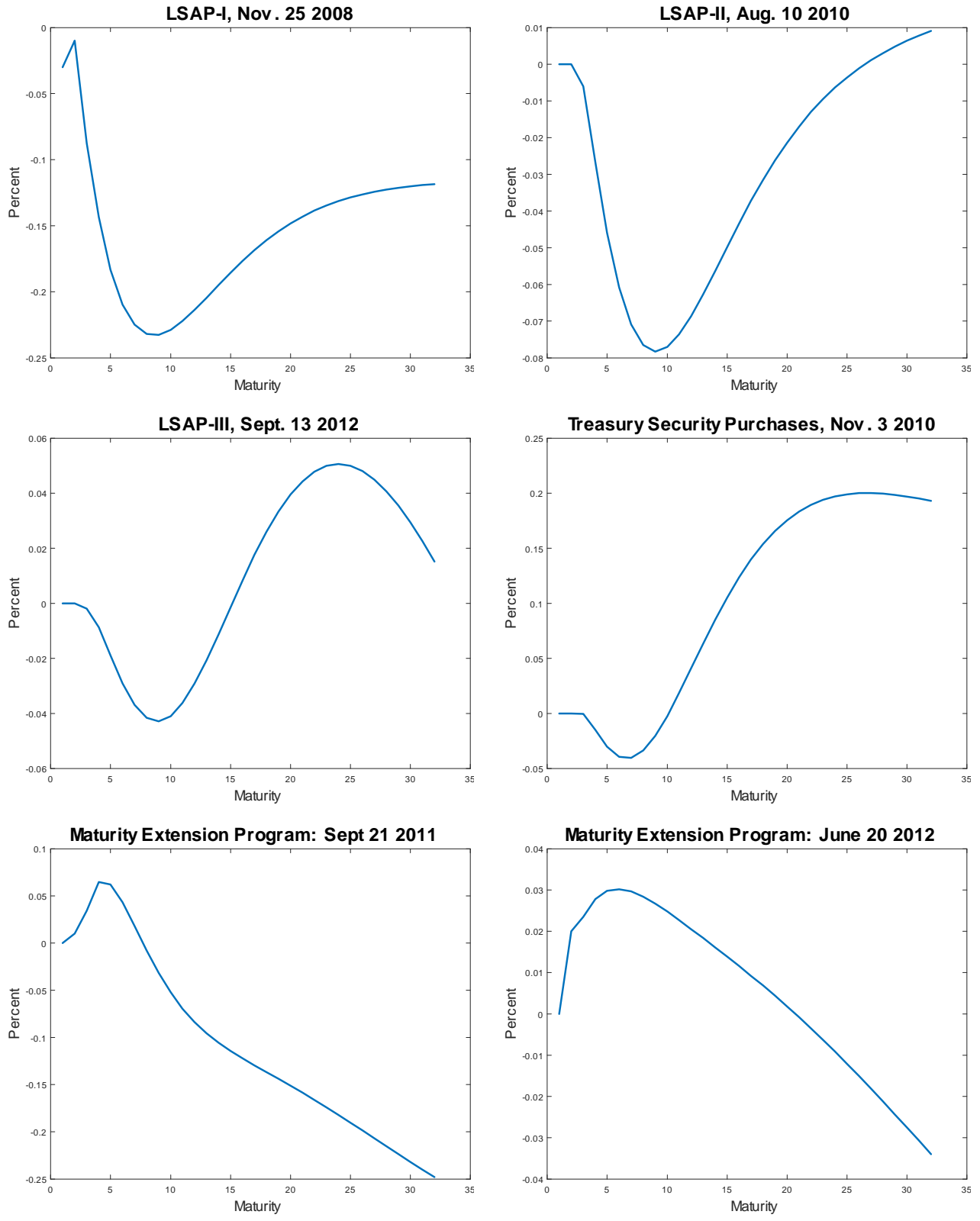


\section{Figure 2. Response to Monetary Policy Shocks: Conventional Period}

\section{Panel A. United Kingdom}
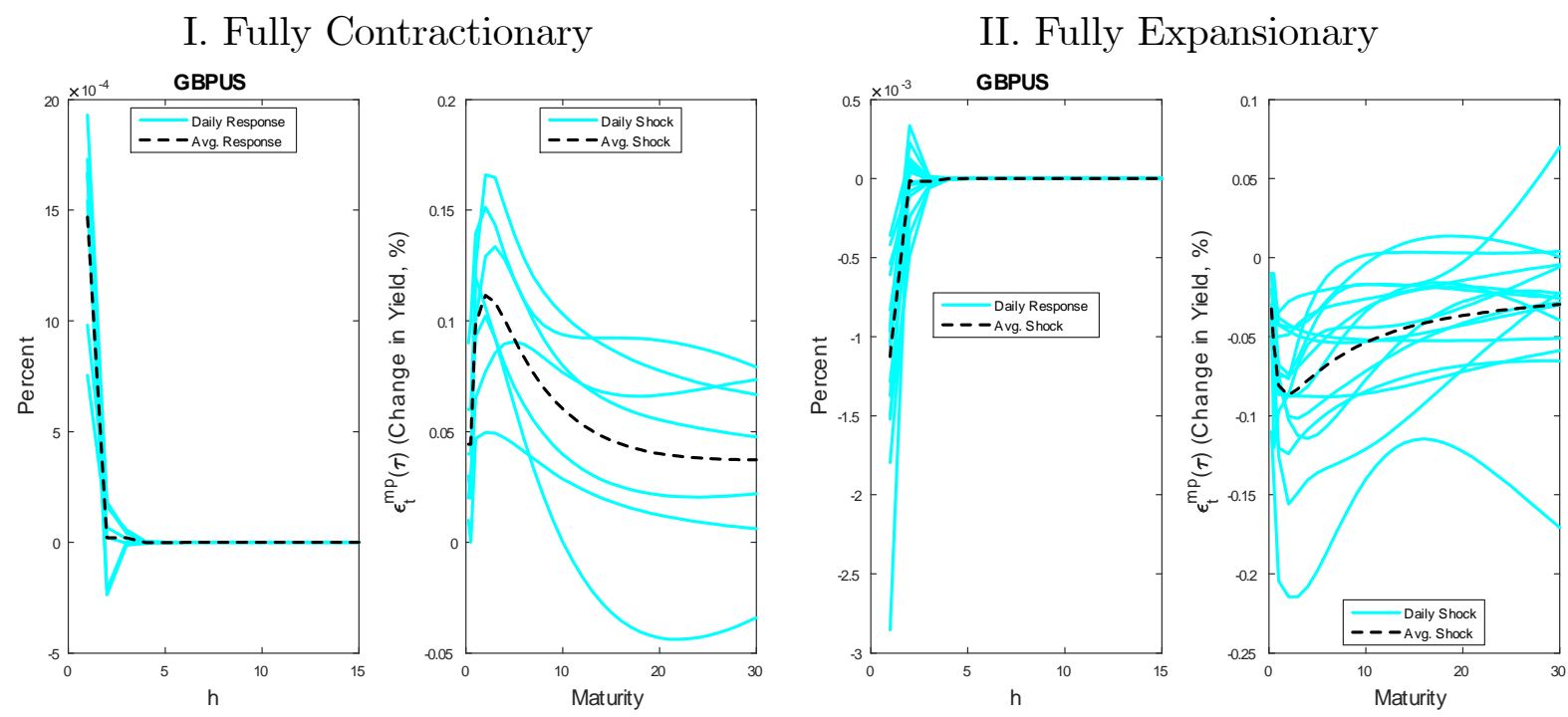

III. More Contractionary at Short
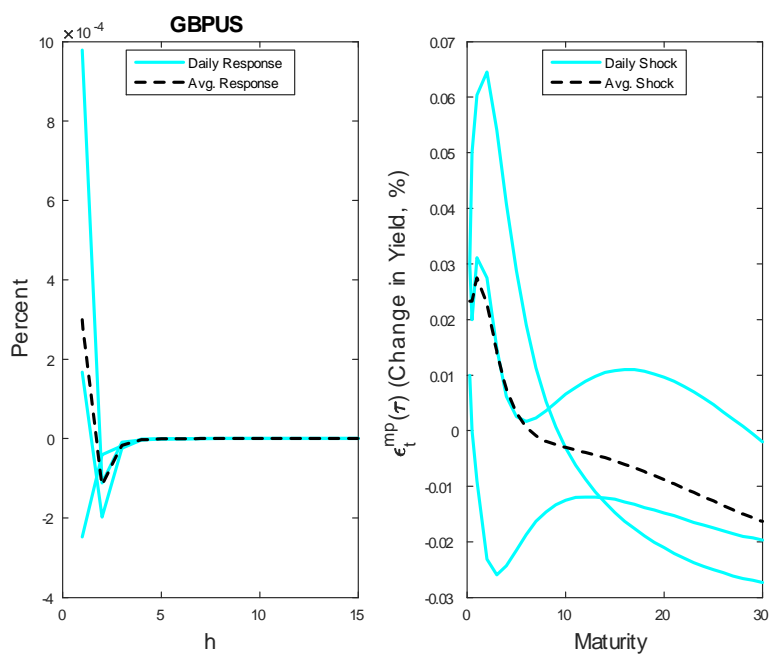

IV. More Expansionary at Short
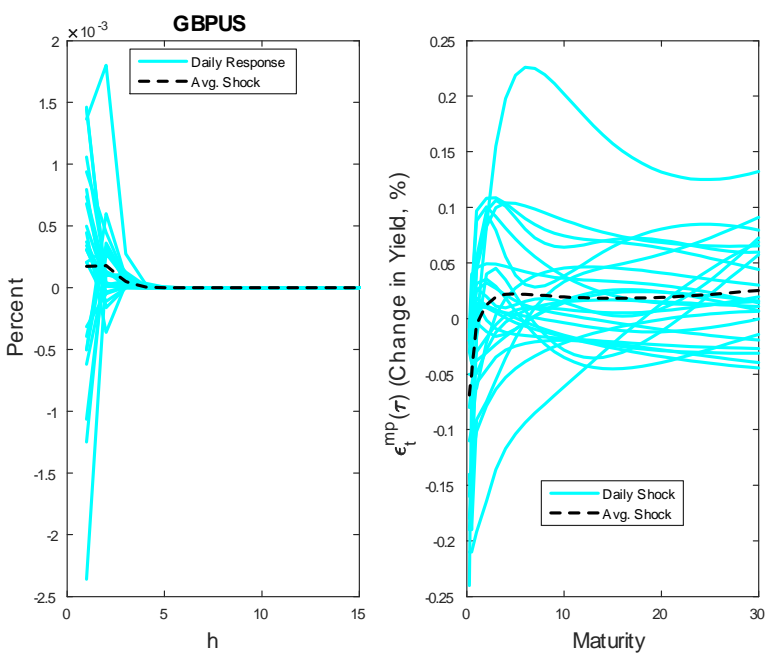
Figure 2 (continued)

\section{Panel B. Euro}
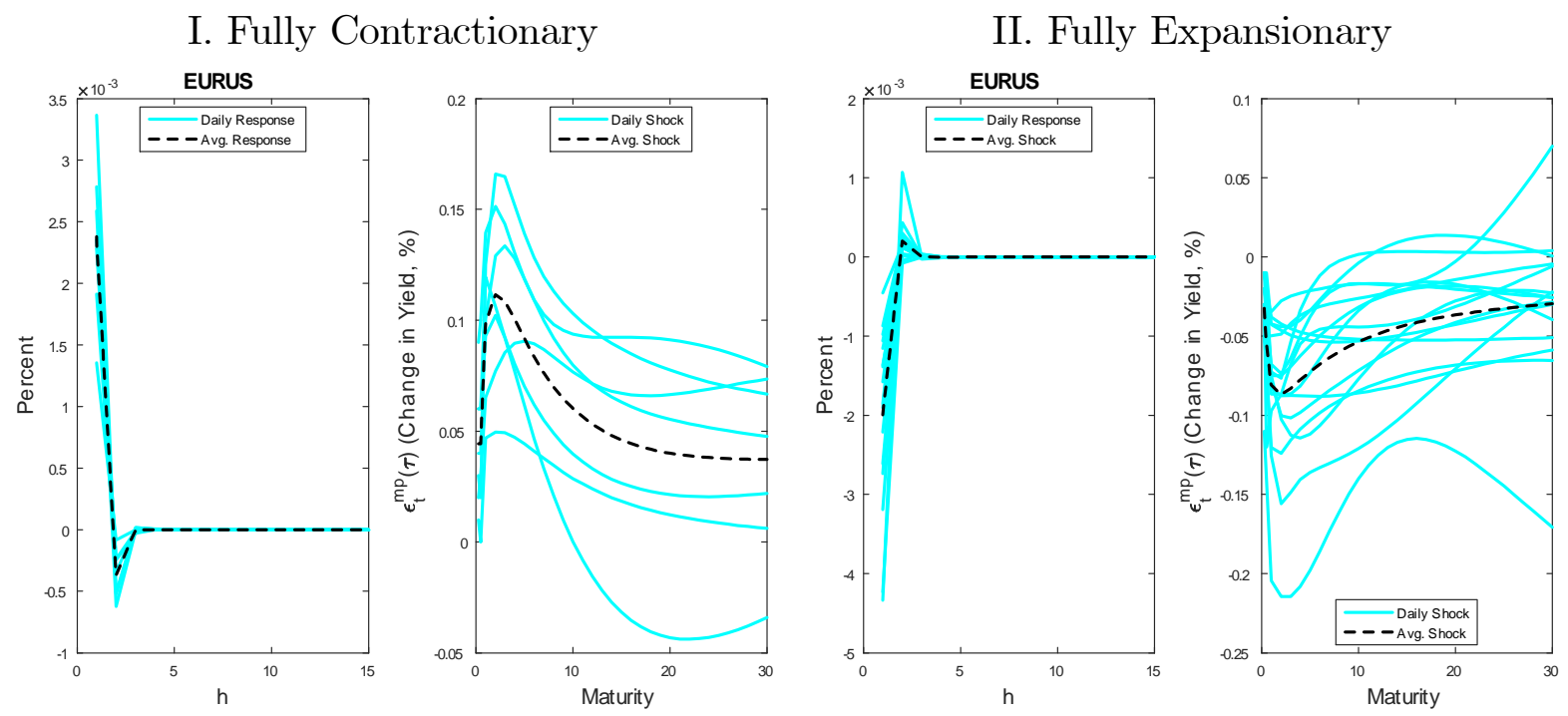

III. More Contractionary at Short
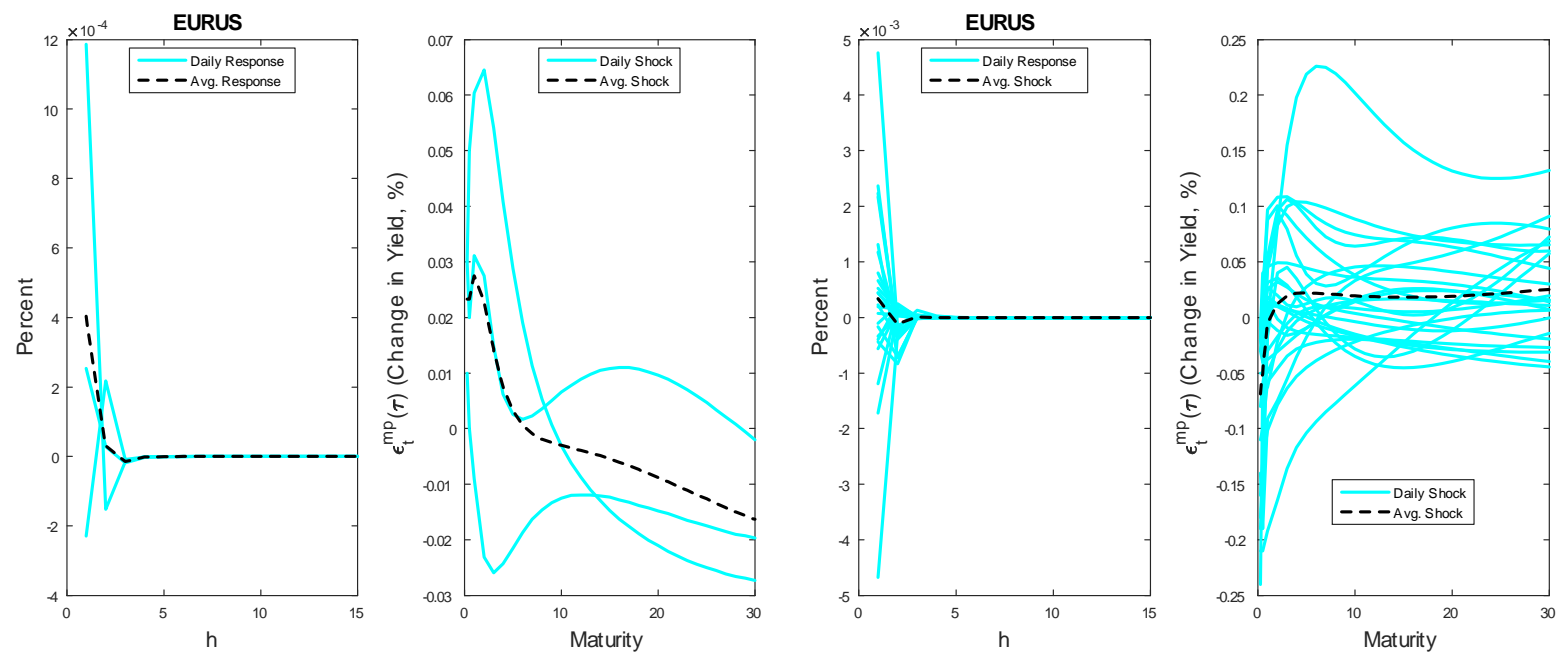
Figure 2 (continued)

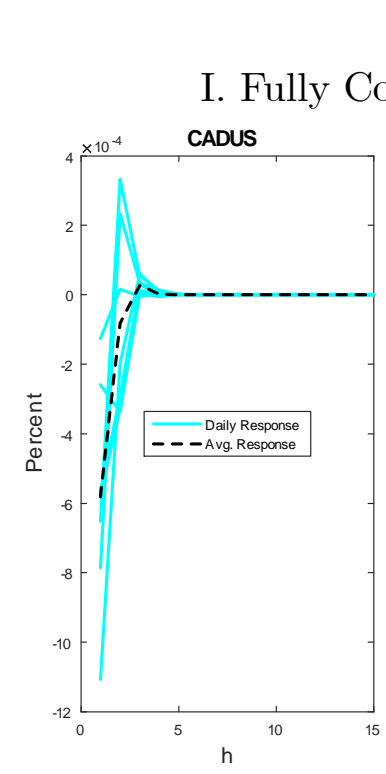

Panel C. Canada
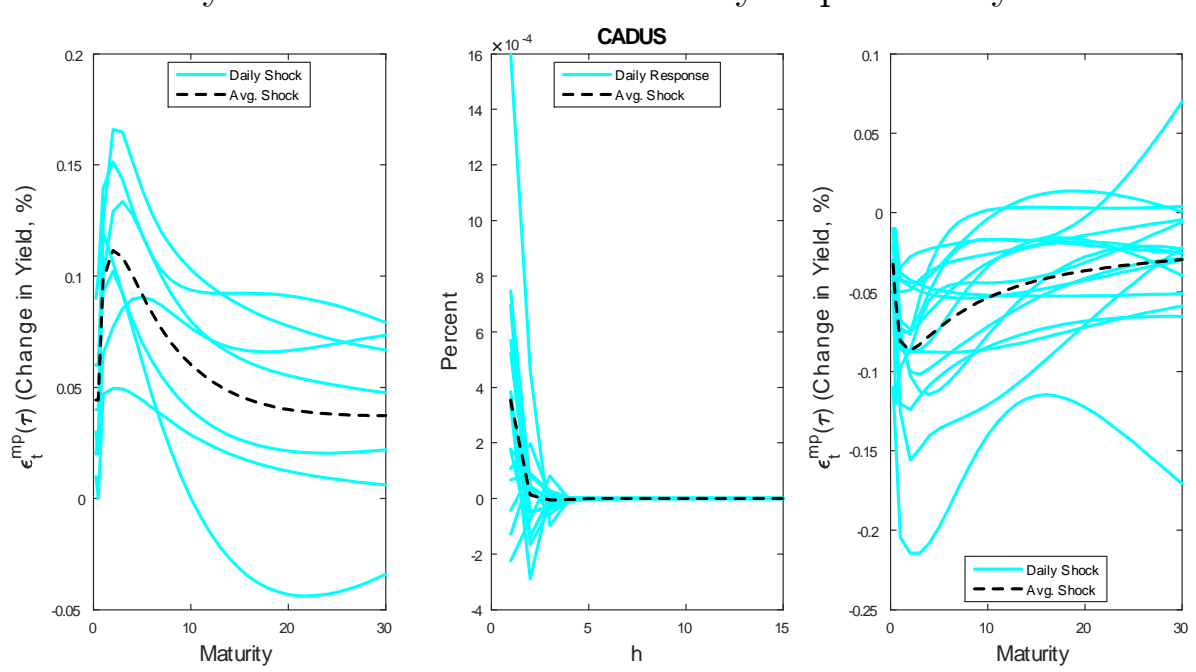

III. More Contractionary at Short
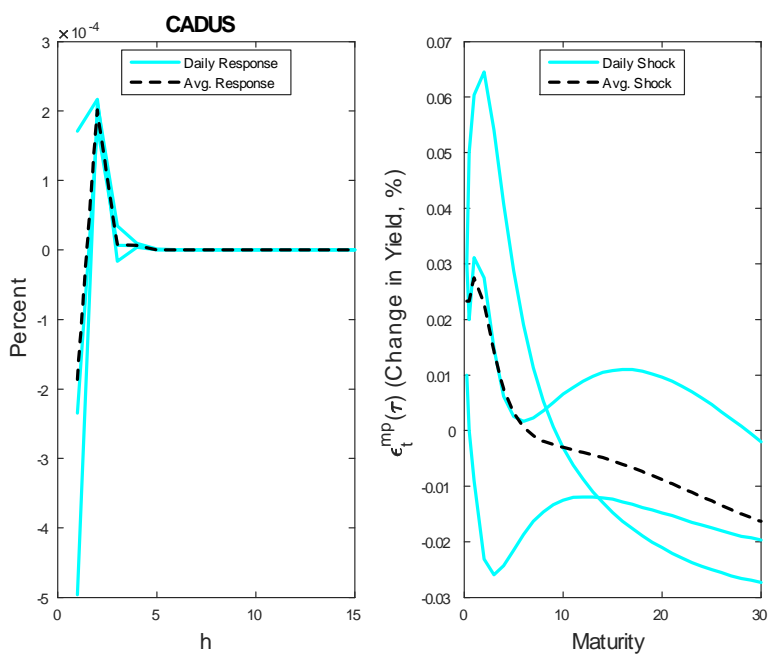

IV. More Expansionary at Short
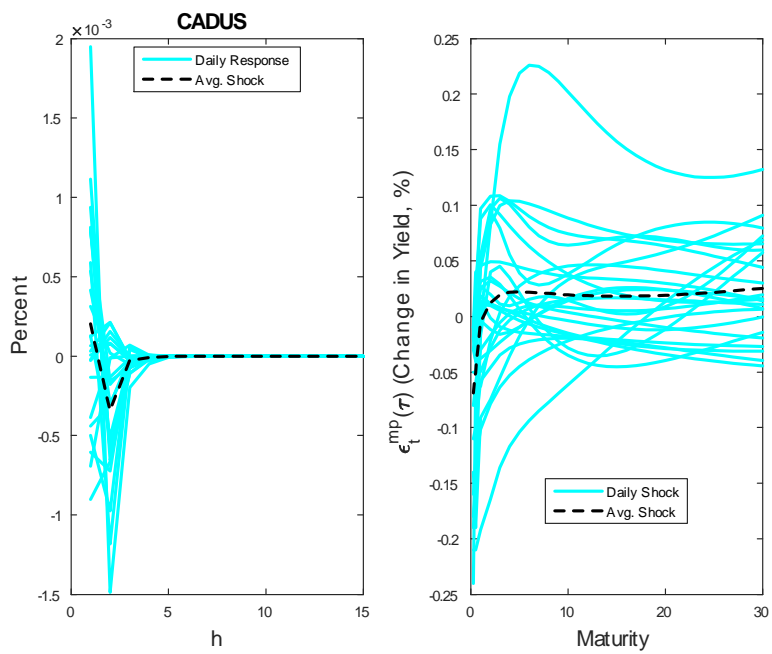
Figure 2 (continued)

Panel D. Japan
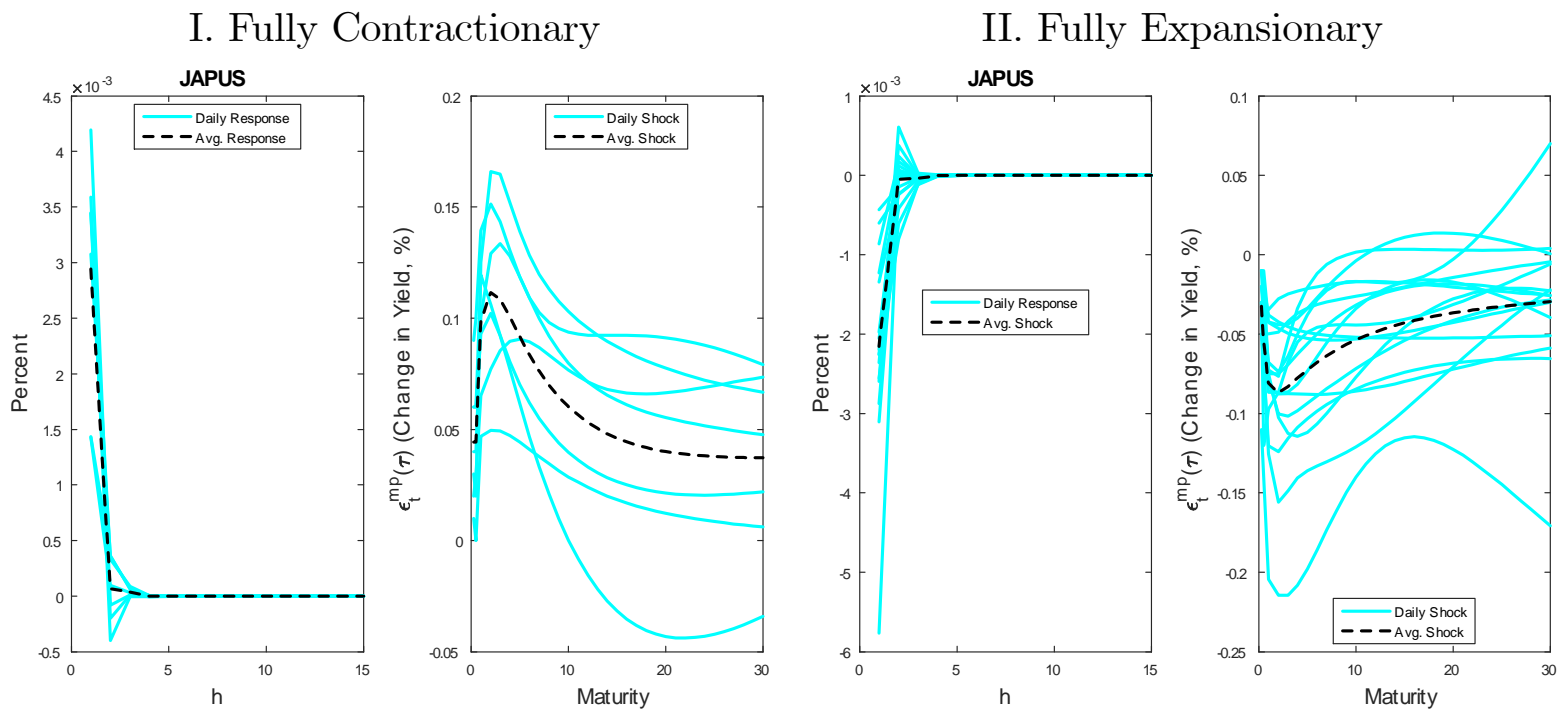

III. More Contractionary at Short
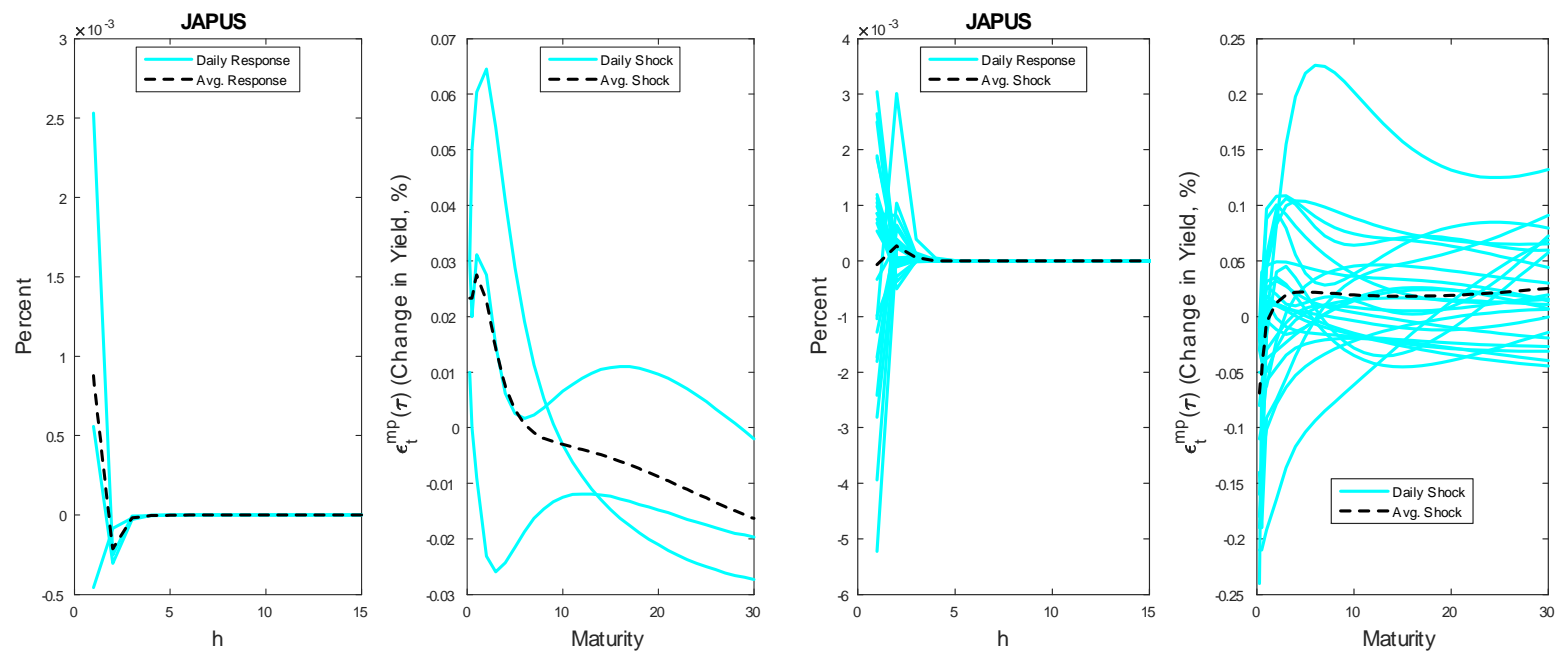

Note to the Figure. "Fully Contractionary" means $\Delta \mathcal{Y}_{1 / 4, t}^{*}>0, \Delta \mathcal{Y}_{5, t}^{*}-\Delta \mathcal{Y}_{3, t}^{*}>0$ and "Fully Expansionary" means $\Delta \mathcal{Y}_{1 / 4, t}^{*}<0, \Delta \mathcal{Y}_{5, t}^{*}-\Delta \mathcal{Y}_{1 / 4, t}^{*}<0$. "More Contractionary at Short" means $\Delta \mathcal{Y}_{1 / 4, t}^{*}<0, \Delta \mathcal{Y}_{5, t}^{*}-\Delta \mathcal{Y}_{3, t}^{*}>0$ while "Less Expansionary at Long" means $\Delta \mathcal{Y}_{1 / 4, t}^{*}>0, \Delta \mathcal{Y}_{5, t}^{*}-\Delta \mathcal{Y}_{1 / 4, t}^{*}<0$. 


\section{Figure 3. Responses to Traditional Monetary Policy}

\section{Shocks in the Conventional Period}

Panel A. United Kingdom
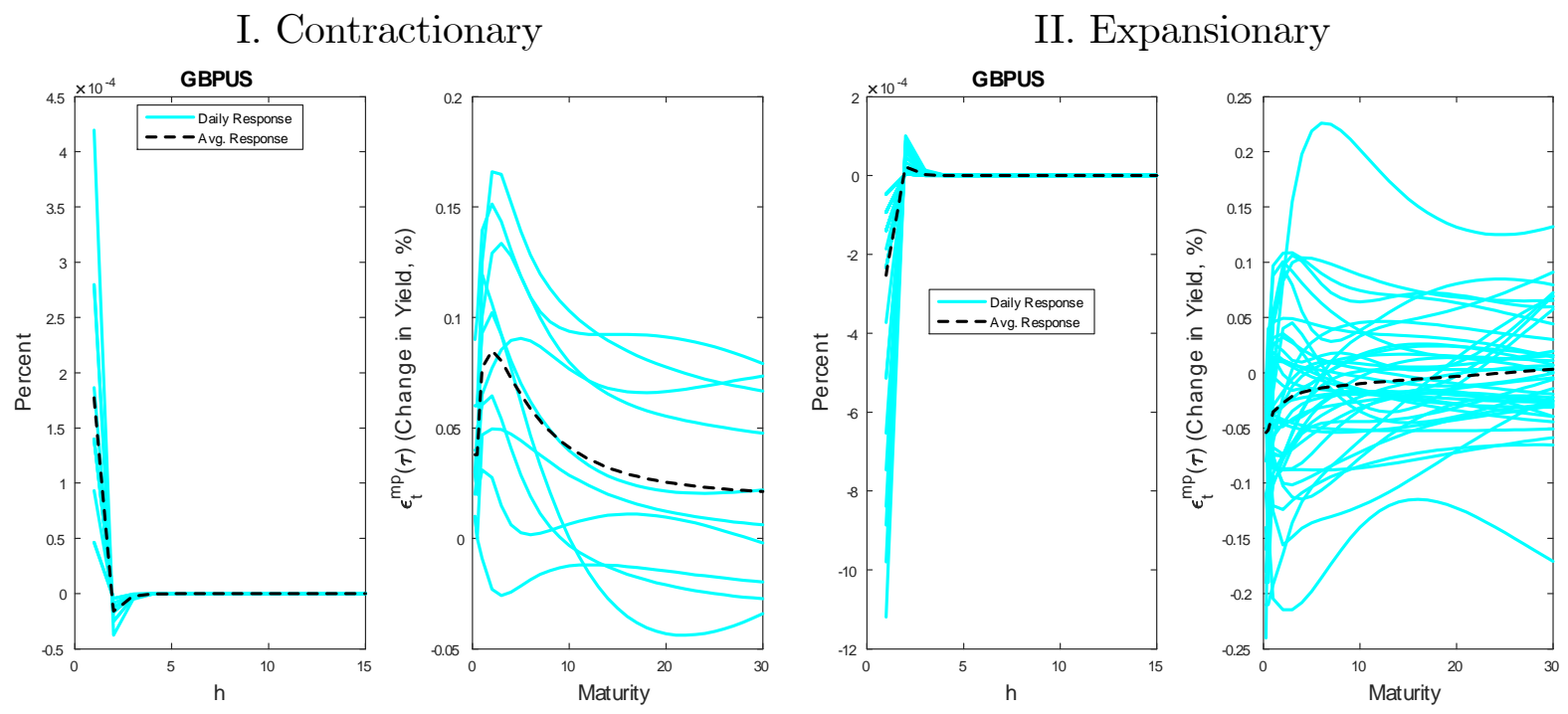

Panel B. Europe

I. Contractionary

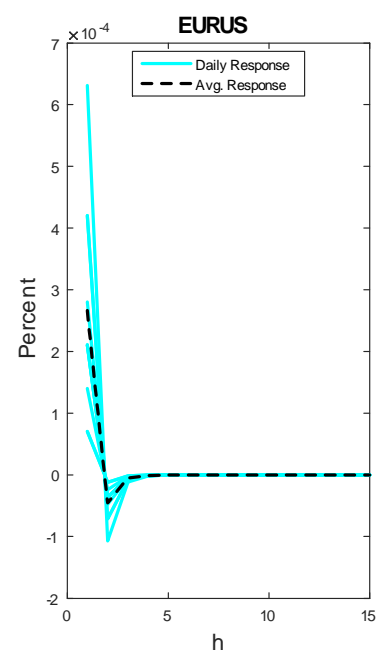

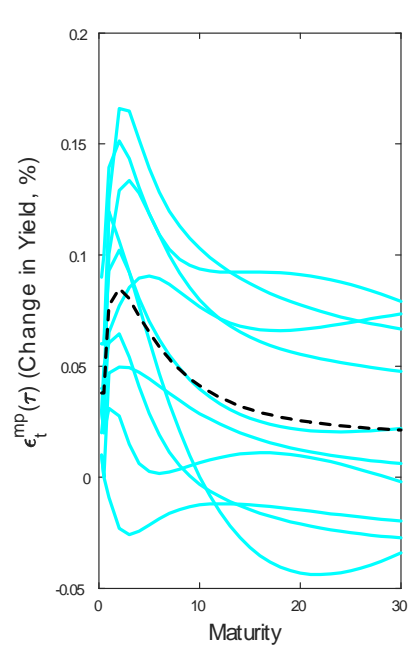

II. Expansionary
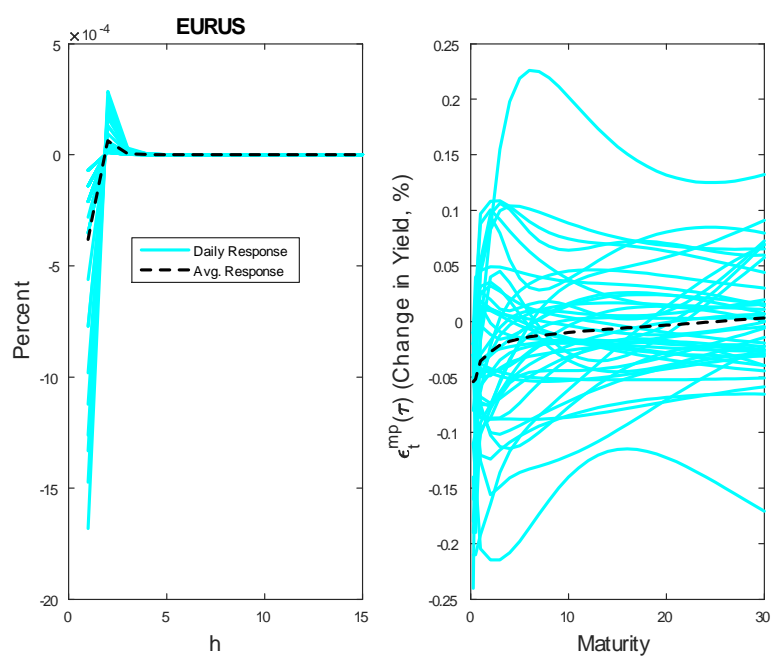
Figure 3 (continued)

\section{Panel C. Canada}
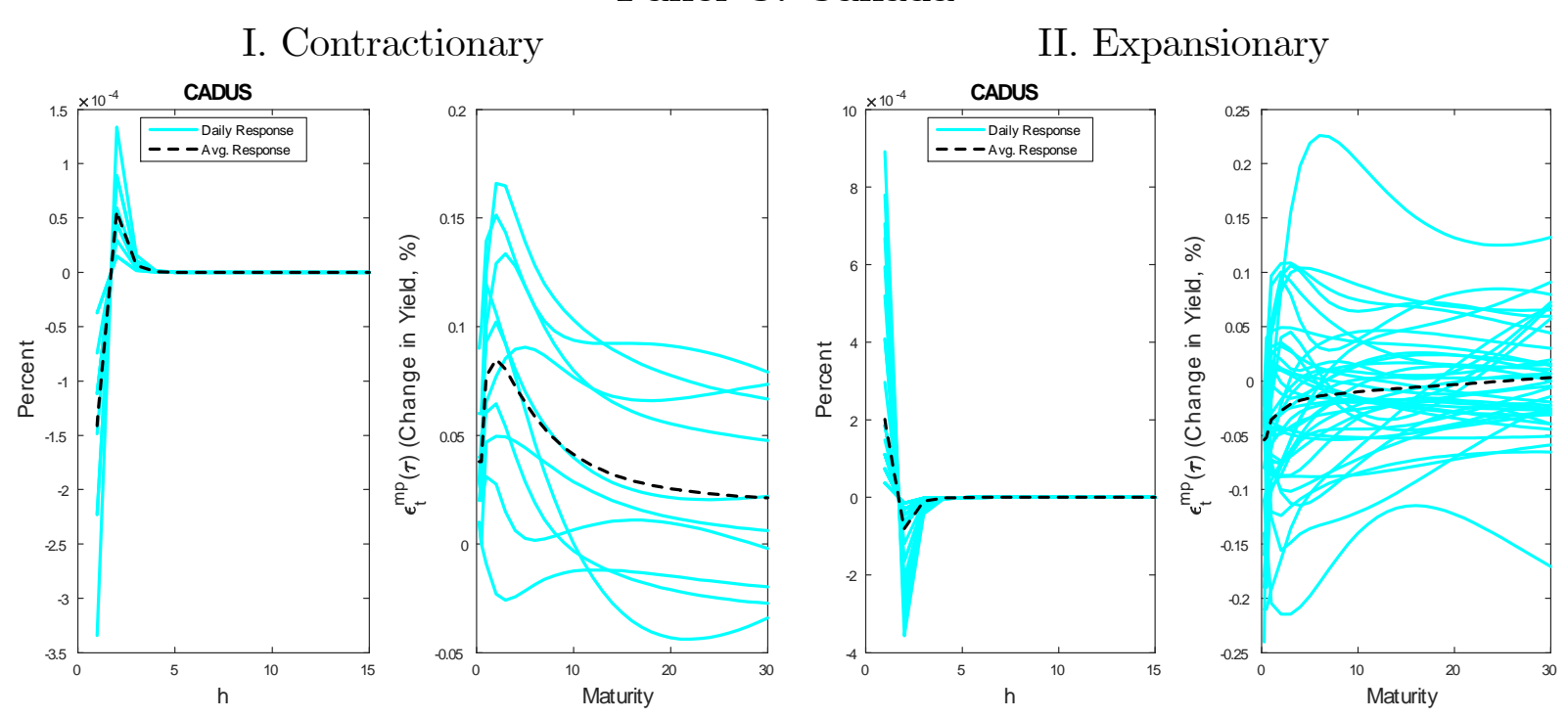

Panel D. Japan
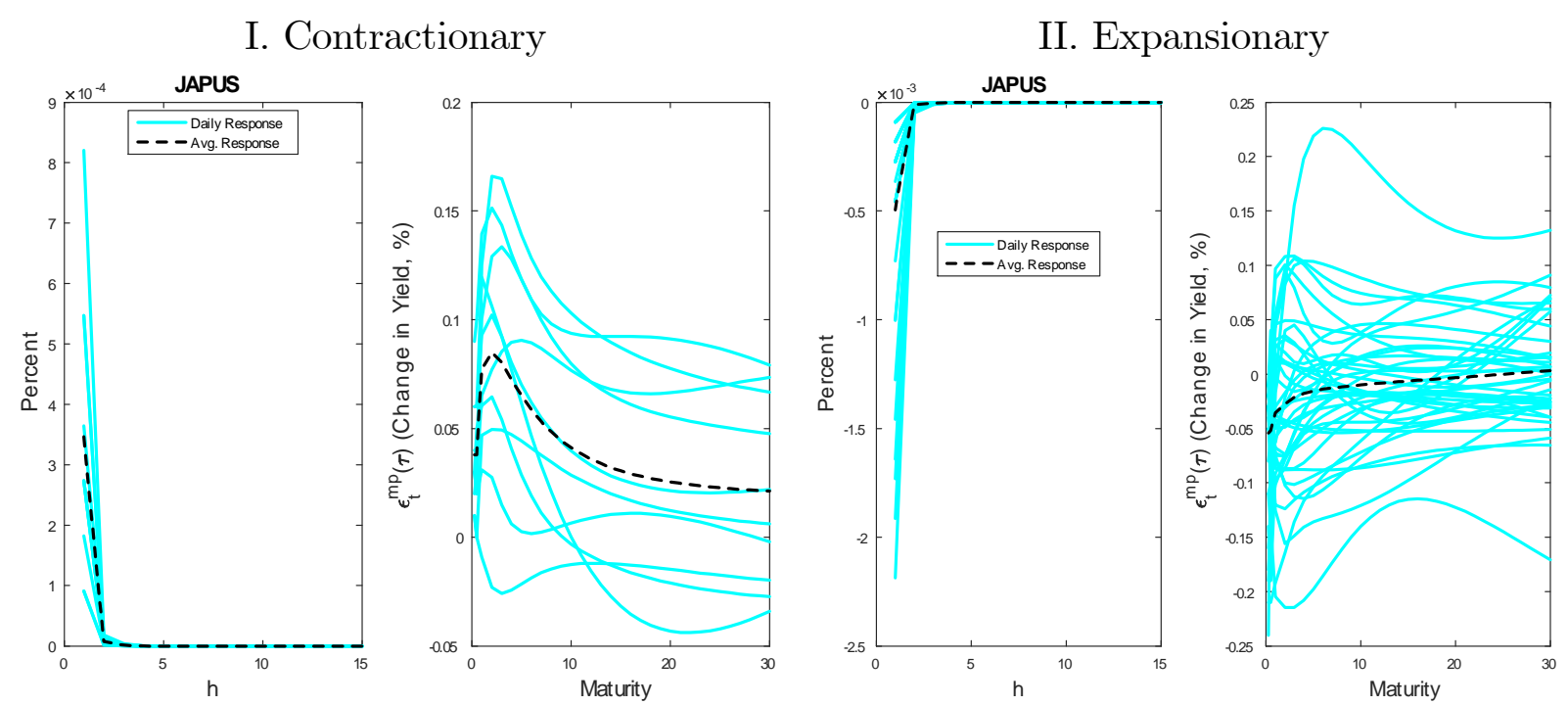

Notes to the figure. Each of the eight figures plots the monetary policy shock (panel on the right) and the corresponding exchange rate's response (panel on the left) for the currencies indicated in the title. The monetary policy shocks are selected to be contractionary (Panel A) and expansionary (Panel B) at the shortest maturity. "Contractionary" means $\Delta \mathcal{Y}_{1 / 4, t}^{*}>0$ and "Expansionary" means $\Delta \mathcal{Y}_{1 / 4, t}^{*}<0$. 
Figure 4. Response to Monetary Policy Shocks: Unconventional Period Panel A. United Kingdom
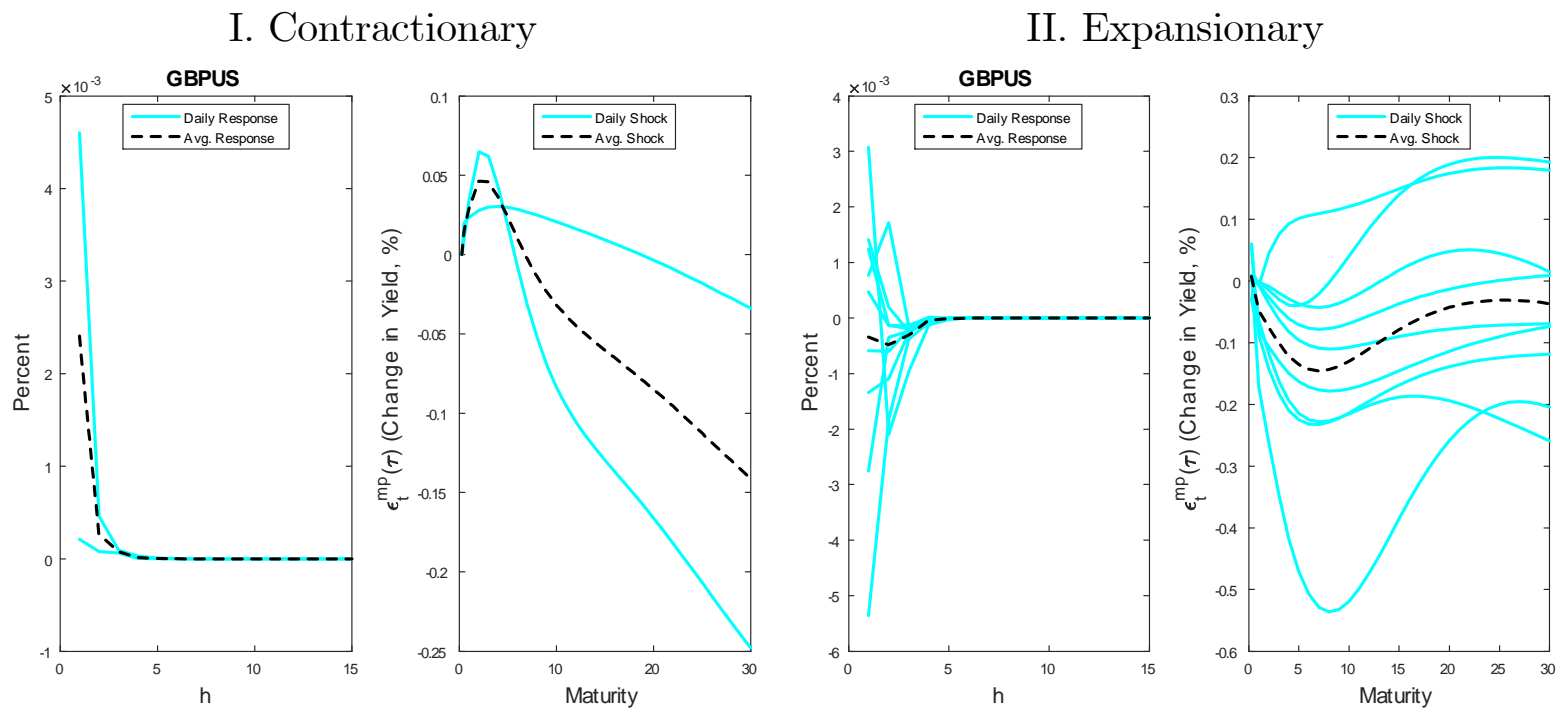

Panel B. Europe
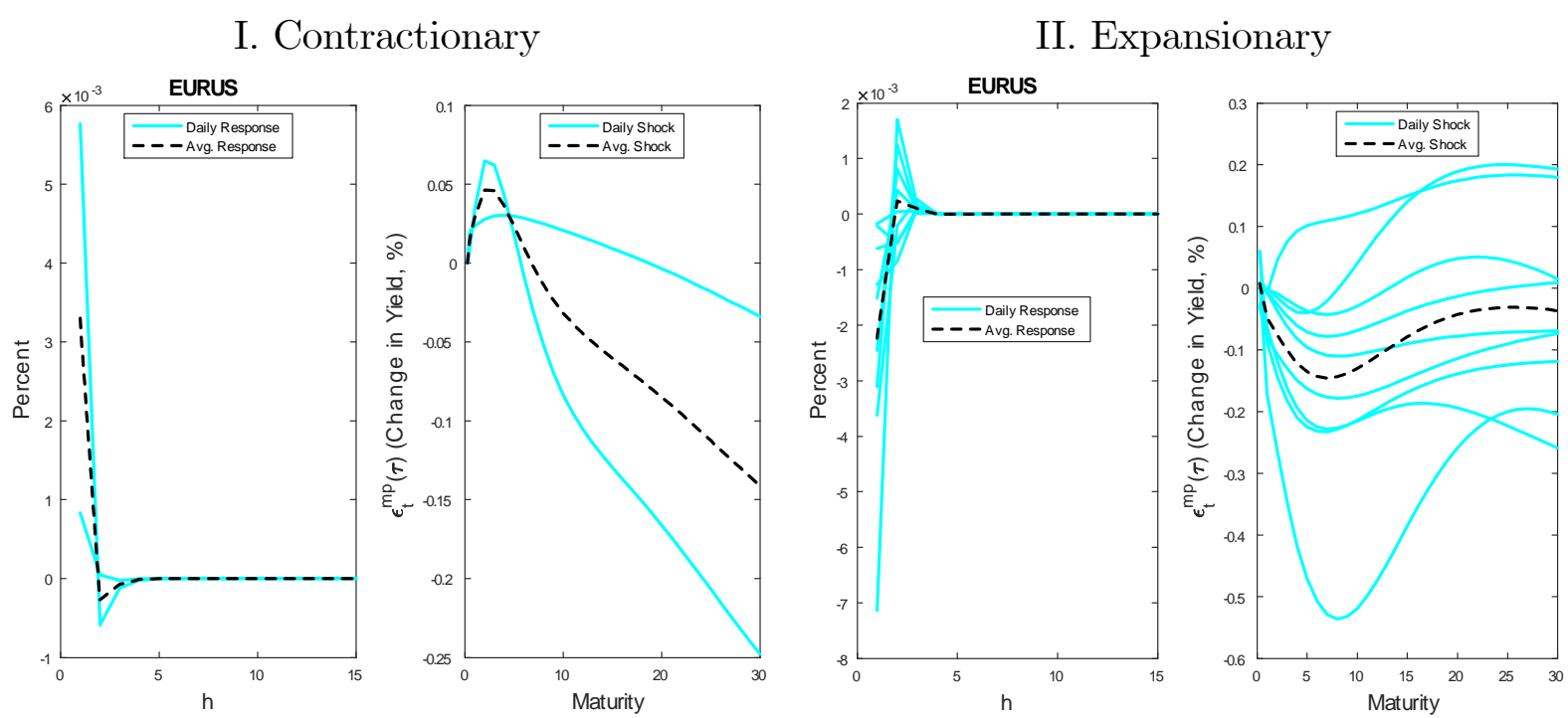
Figure 4 (continued)

Panel C. Canada
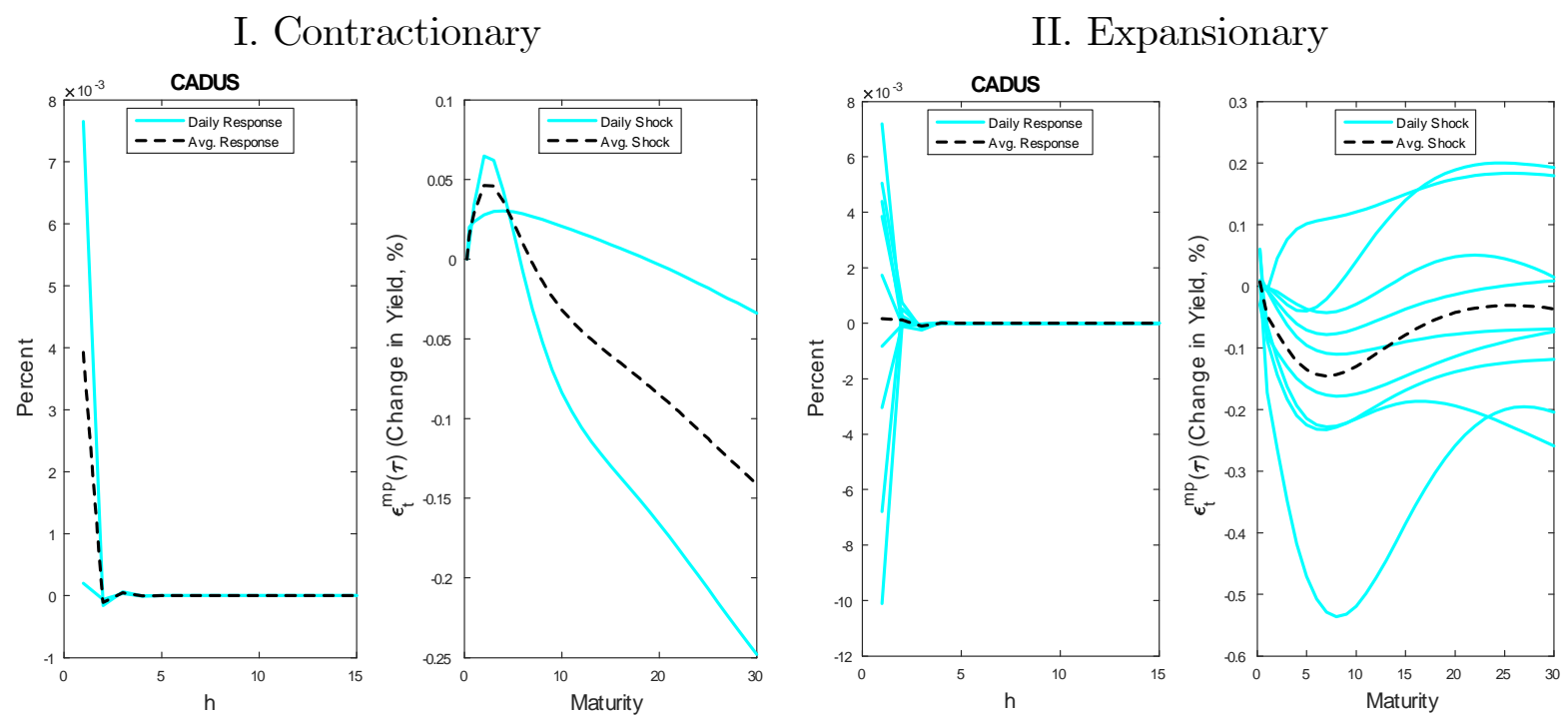

Panel D. Japan
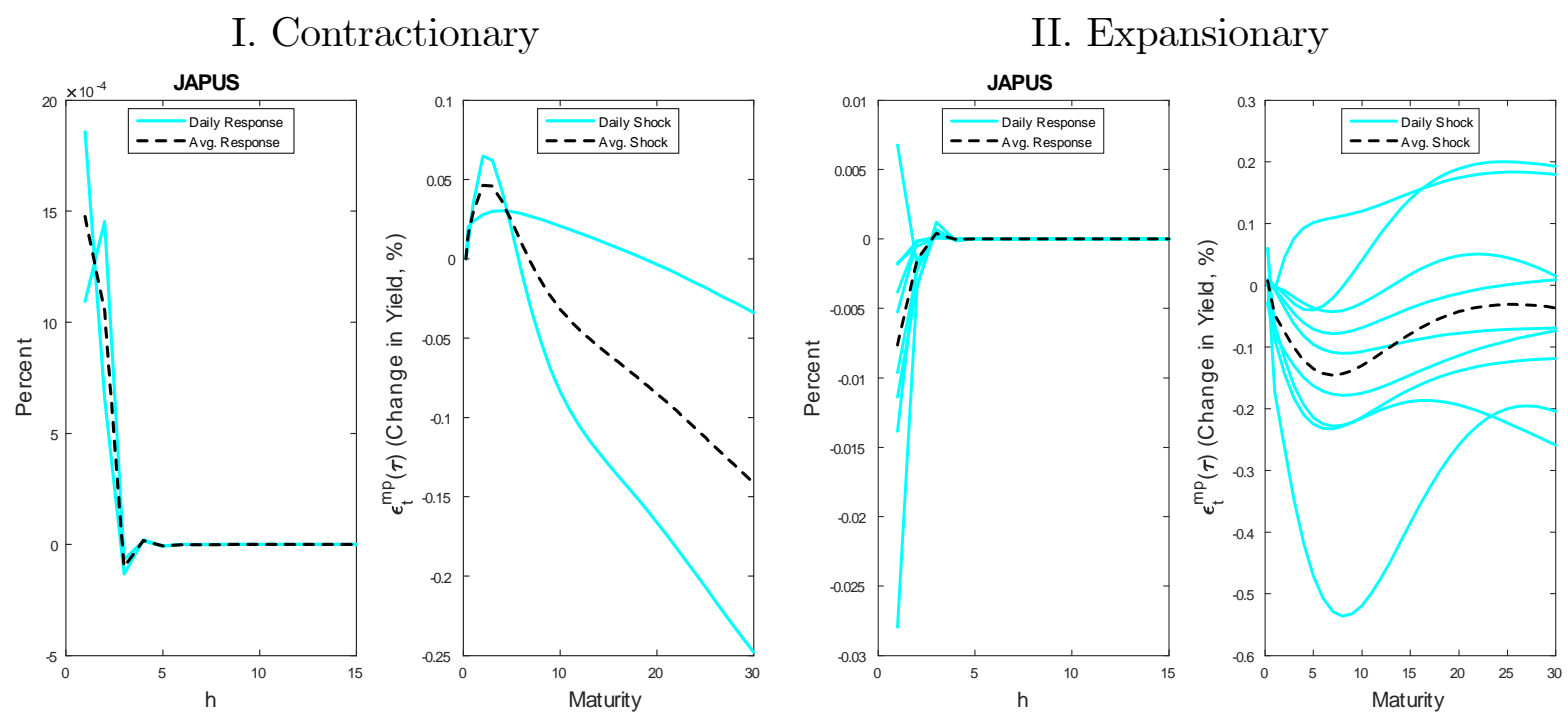

Note to the figure. Each of the four figures plots the monetary policy shock (panel on the right) and the corresponding exchange rate's response (panel on the left) for the currencies indicated in the title. "Contractionary" means $\Delta \mathcal{Y}_{5, t}^{*}>0$ and "Expansionary" means $\Delta \mathcal{Y}_{5, t}^{*}<0$ for all countries. 
Figure 5. Response to Monetary Policy Shocks
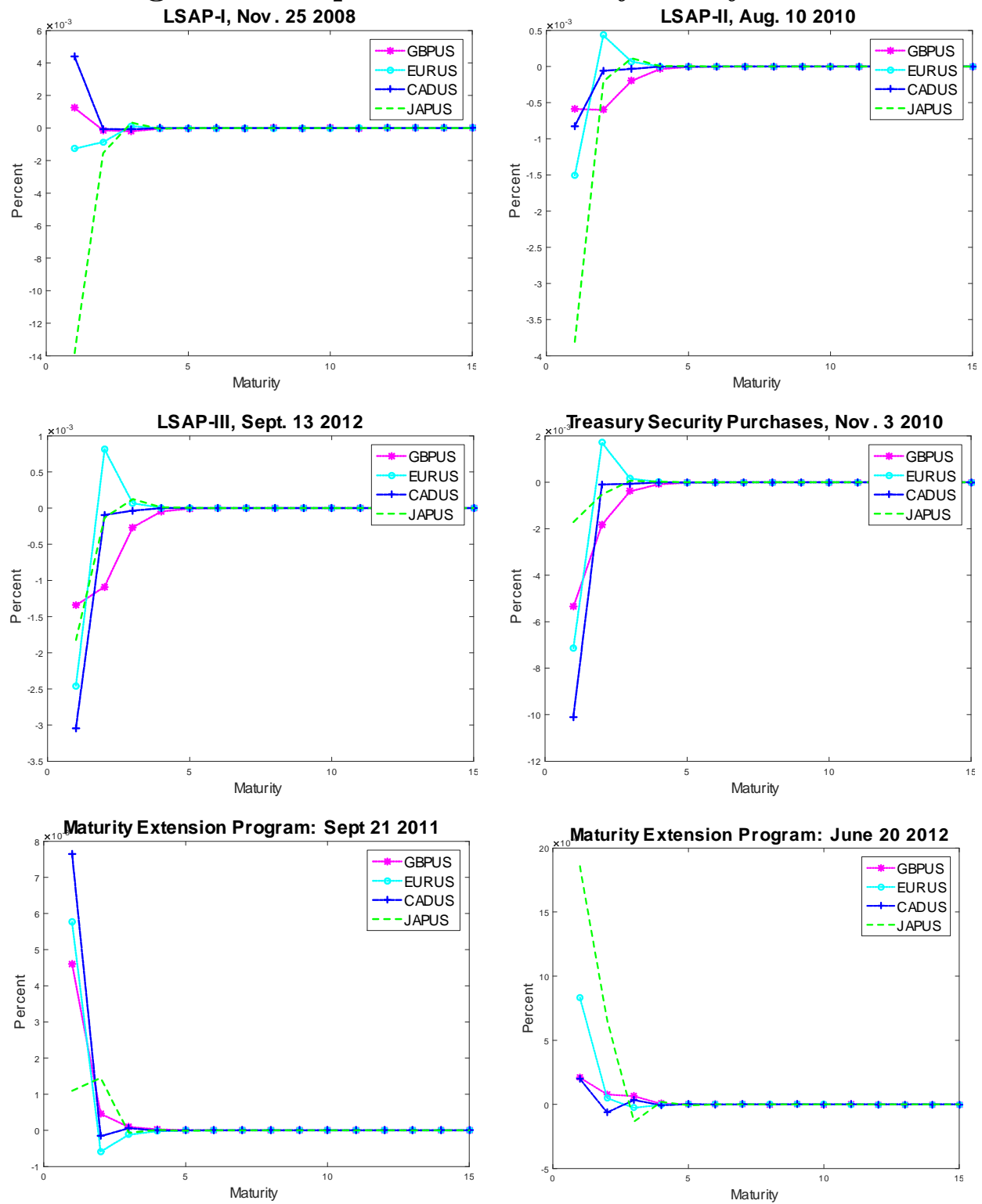

Note. The figure plots responses of exchange rates on selected announcement dates. 


\section{Figure 6. Response to Monetary Policy Shocks Robust to Informational}

\section{Effects: Conventional Period}

\section{Panel A. United Kingdom}
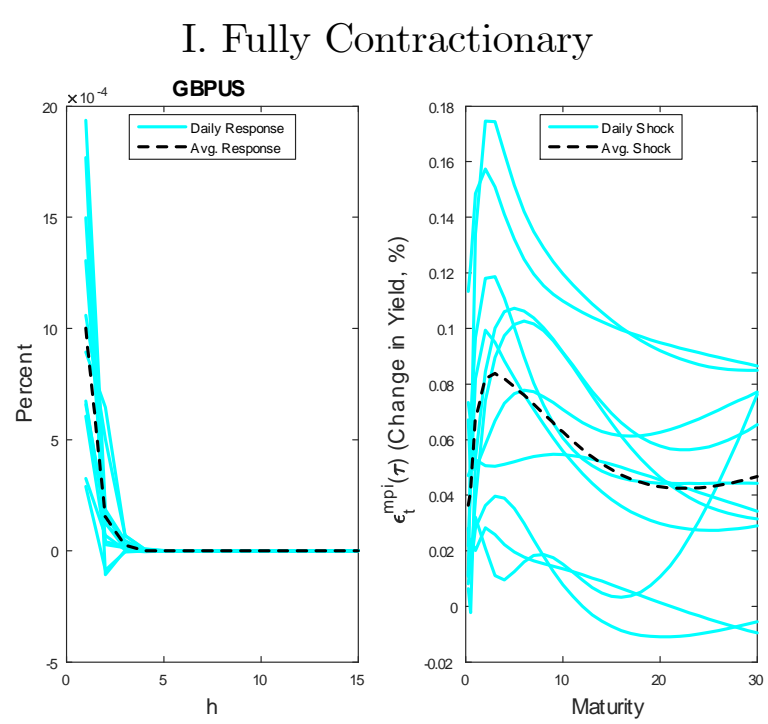

III. More Contractionary at Short

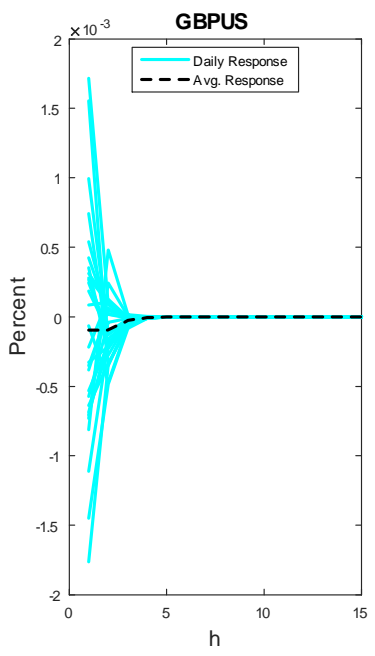

II. Fully Expansionary
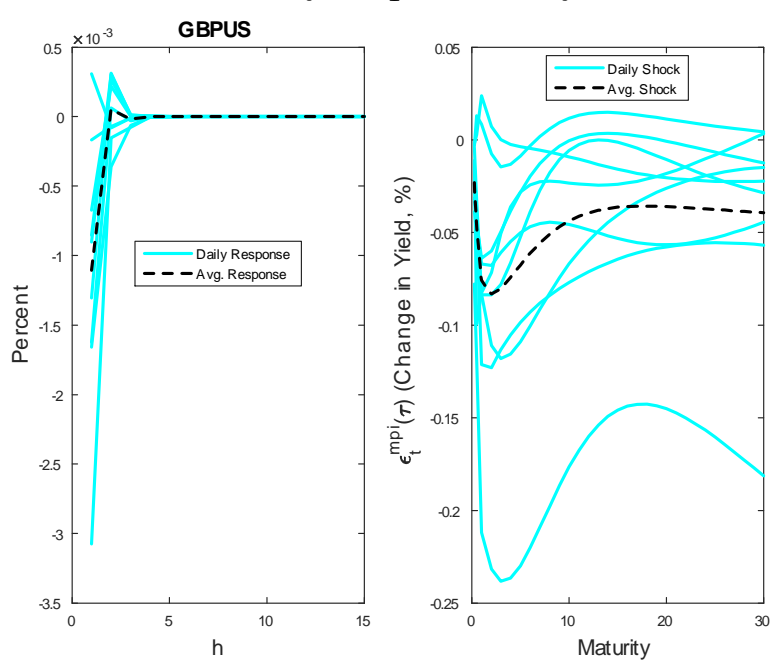

IV. More Expansionary at Short

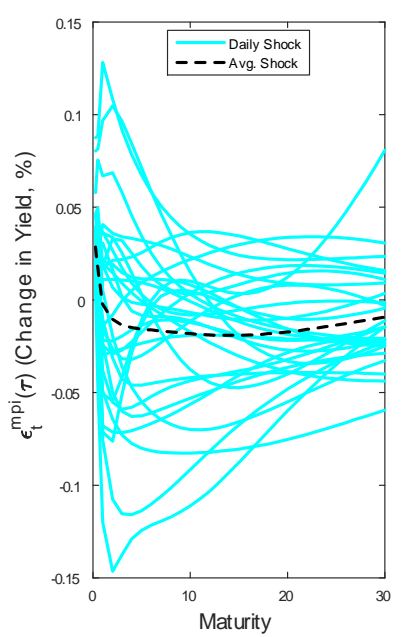

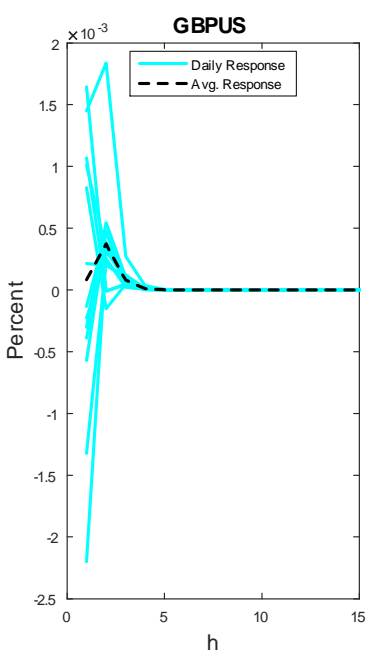

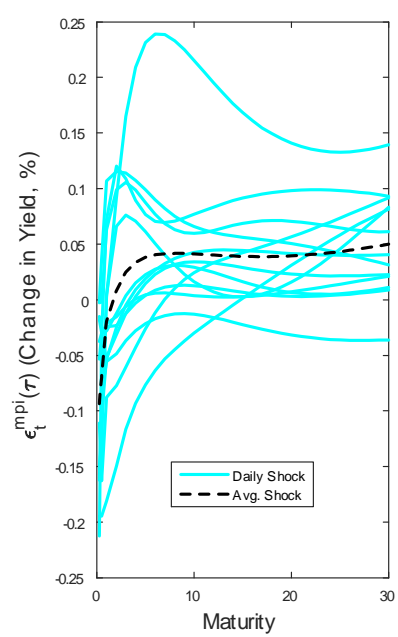




\section{Figure 6 (continued)}

\section{Panel B. Euro}
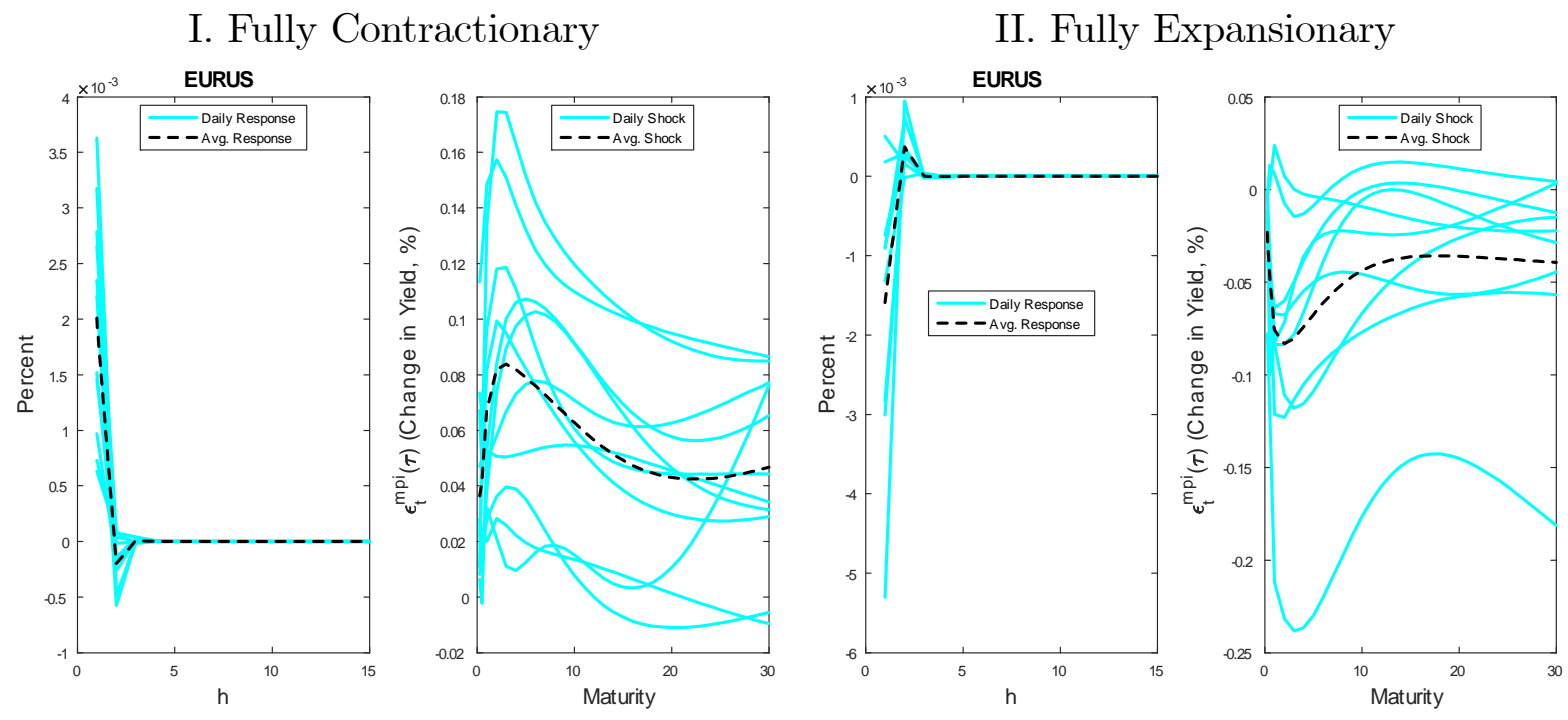

III. More Contractionary at Short
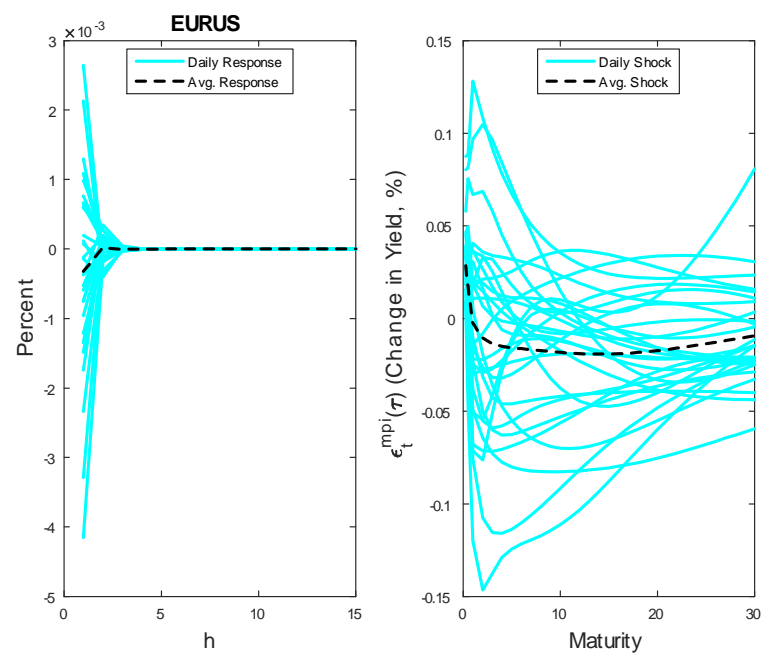

IV. More Expansionary at Short
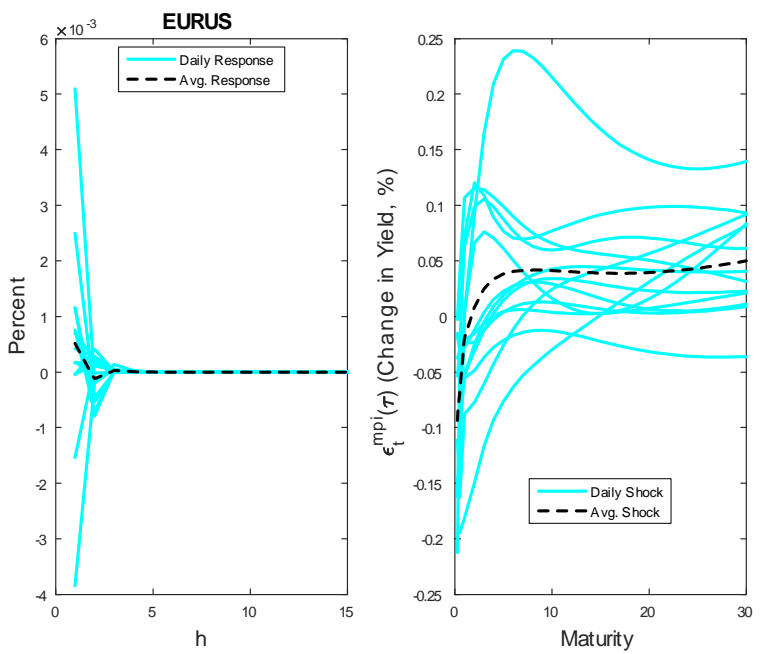


\section{Figure 6 (continued)}

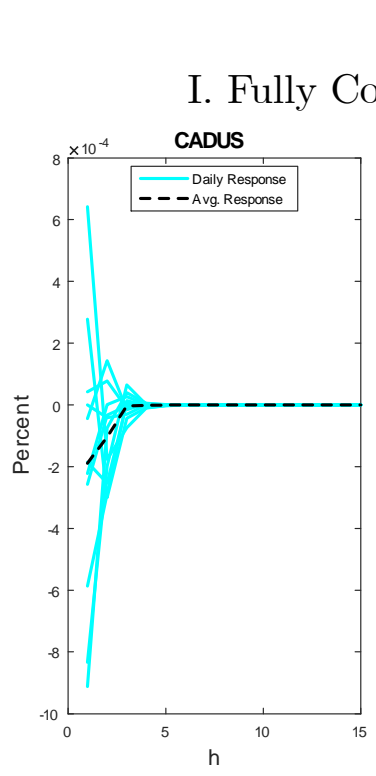

\section{Panel C. Canada}
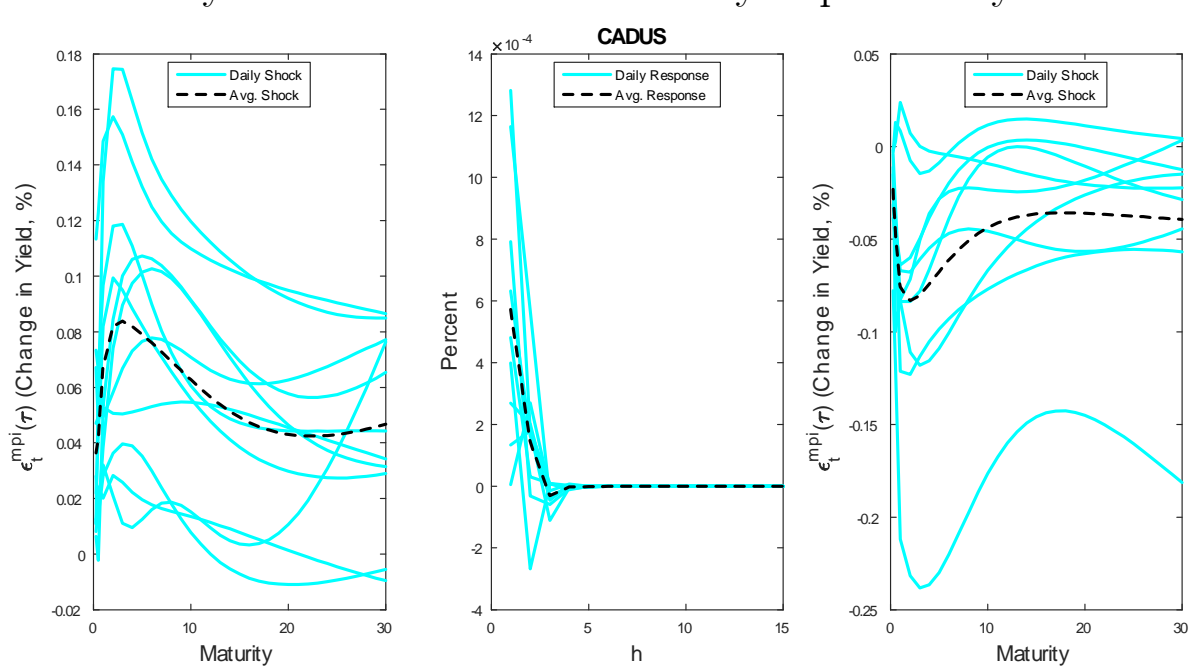

III. More Contractionary at Short
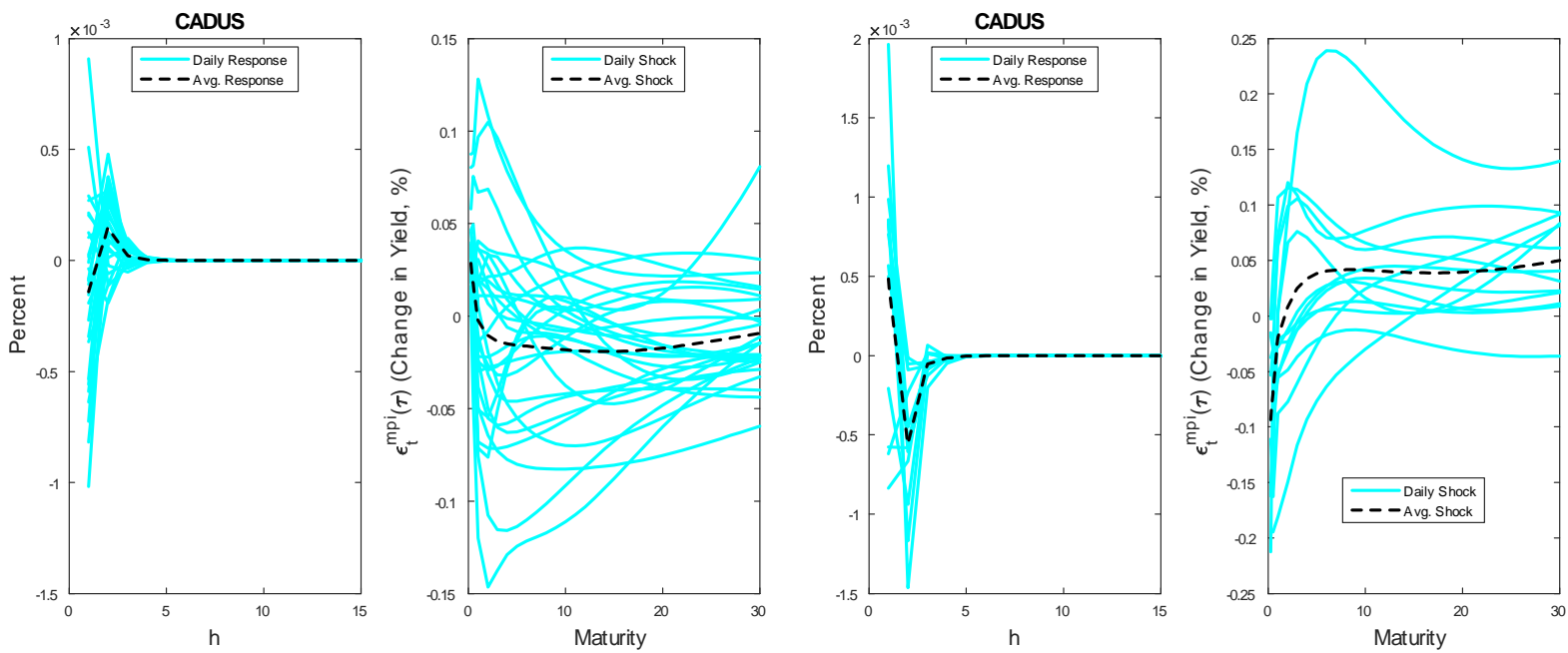


\section{Figure 6 (continued)}

\section{Panel D. Japan}
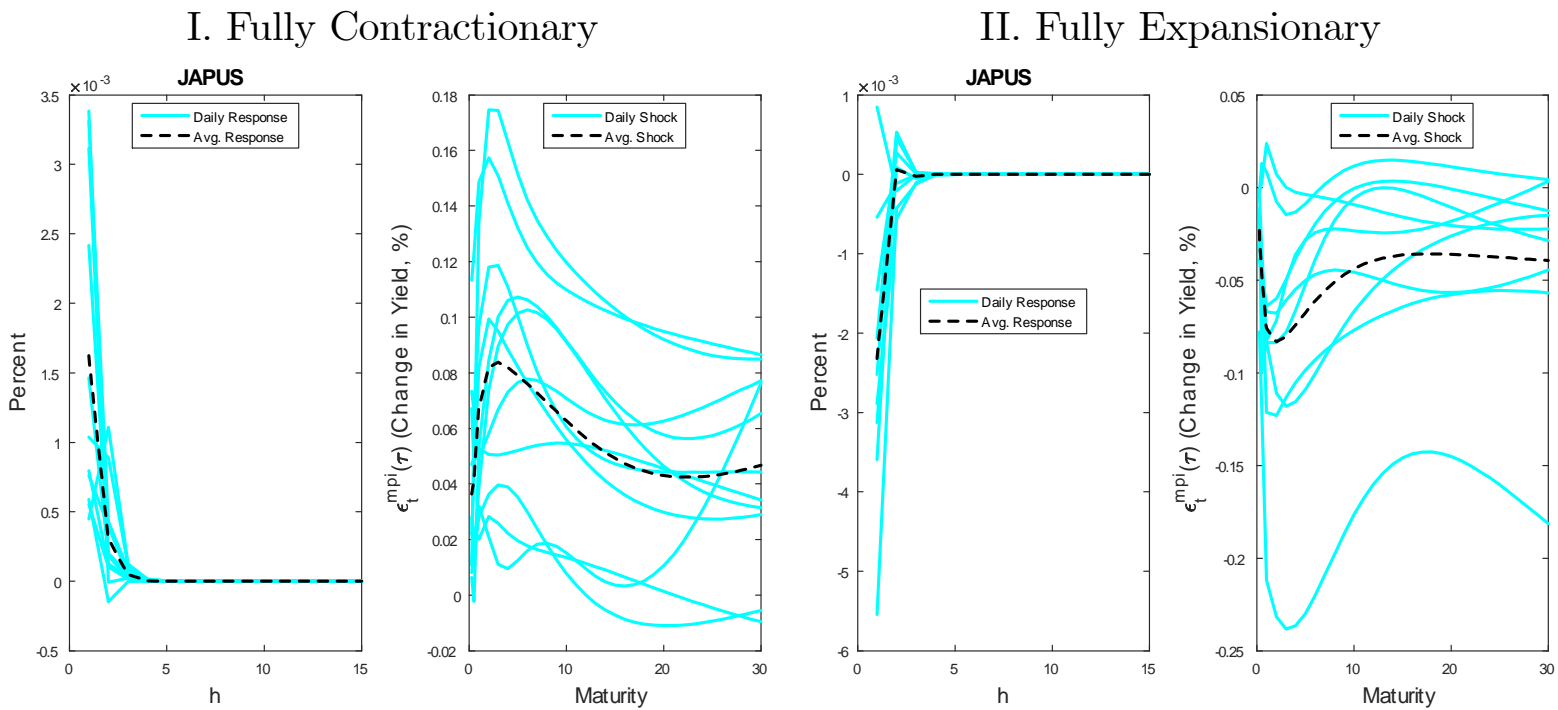

III. More Contractionary at Short
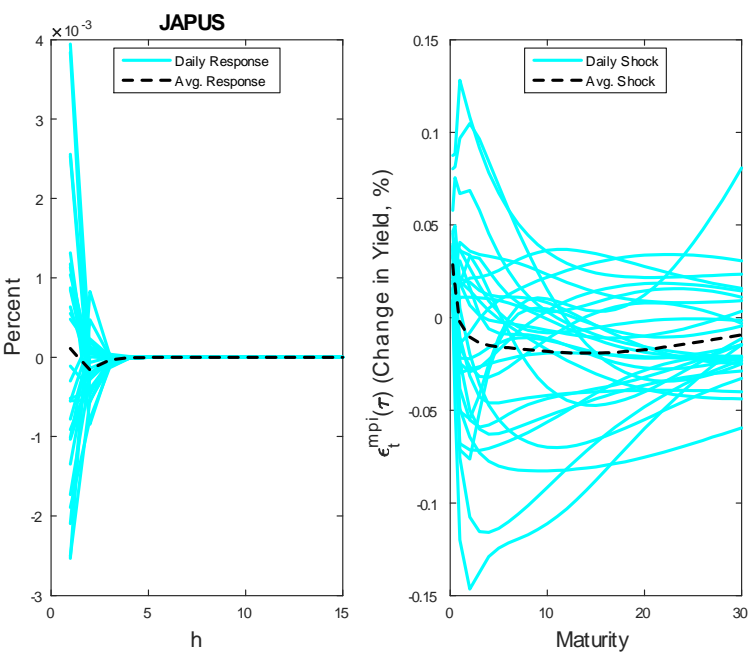

IV. More Expansionary at Short
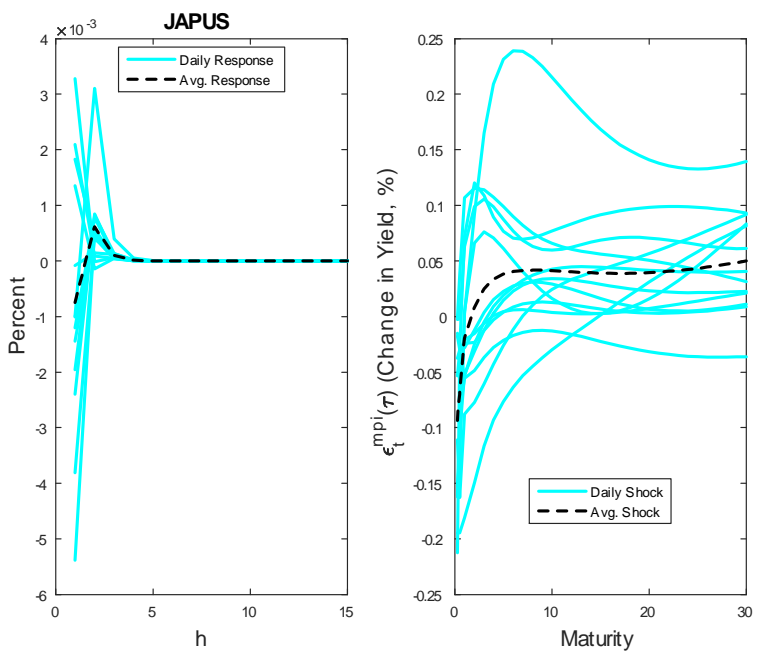

Note to the Figure. "Fully Contractionary" means $\Delta \mathcal{Y}_{1 / 4, t}^{*}>0, \Delta \mathcal{Y}_{5, t}^{*}-\Delta \mathcal{Y}_{1 / 4, t}^{*}>0$ and "Fully Expansionary" means $\Delta \mathcal{Y}_{1 / 4, t}^{*}<0, \Delta \mathcal{Y}_{5, t}^{*}-\Delta \mathcal{Y}_{1 / 4, t}^{*}<0$. "More Contractionary at Short" means $\Delta \mathcal{Y}_{1 / 4, t}^{*}<0, \Delta \mathcal{Y}_{5, t}^{*}-\Delta \mathcal{Y}_{1 / 4, t}^{*}>0$ while "Less Expansionary at Long" means $\Delta \mathcal{Y}_{1 / 4, t}^{*}>0, \Delta \mathcal{Y}_{5, t}^{*}-\Delta \mathcal{Y}_{1 / 4, t}^{*}<0$. 
Figure 7. Response to Real US Monetary Policy Shocks:

\section{Unconventional Period}

\section{Panel A. United Kingdom}
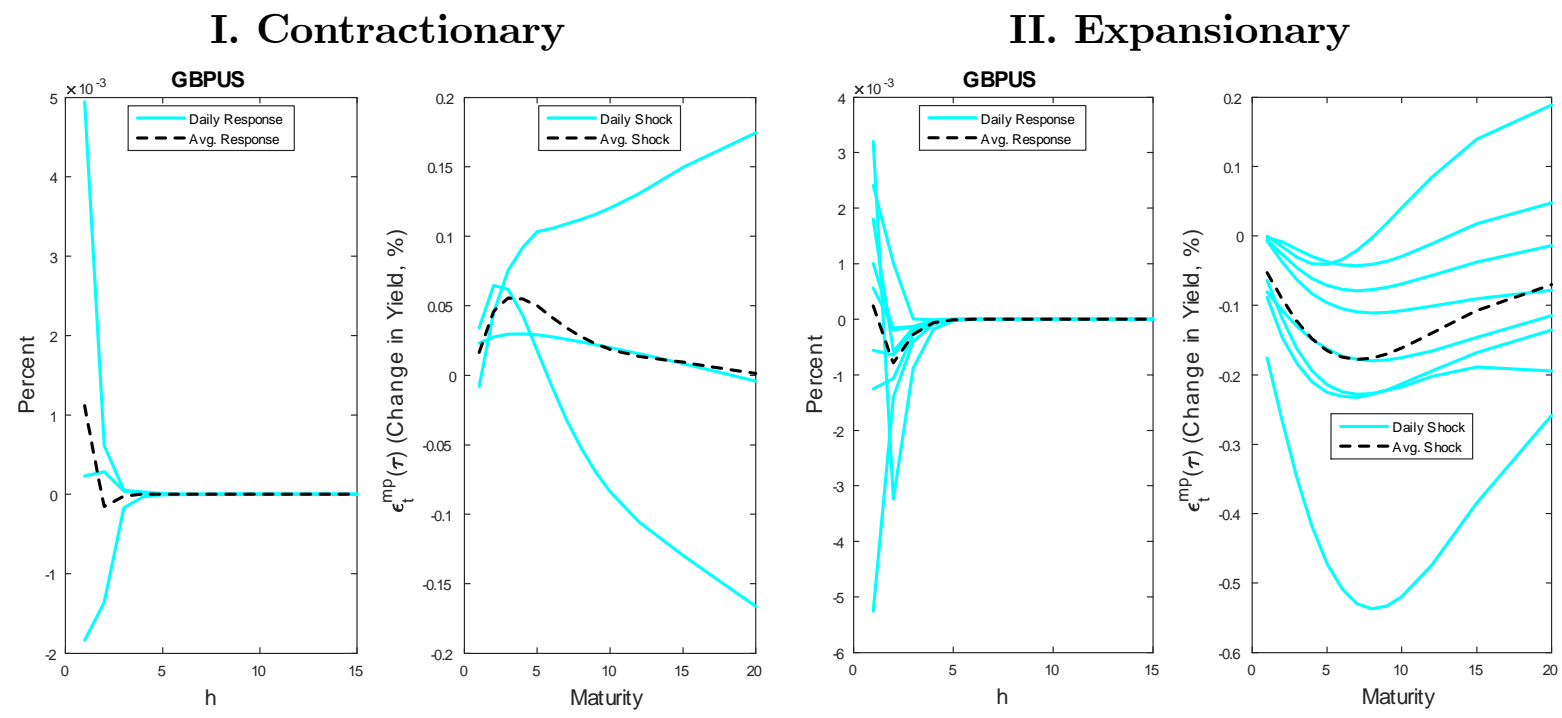

Panel B. Europe
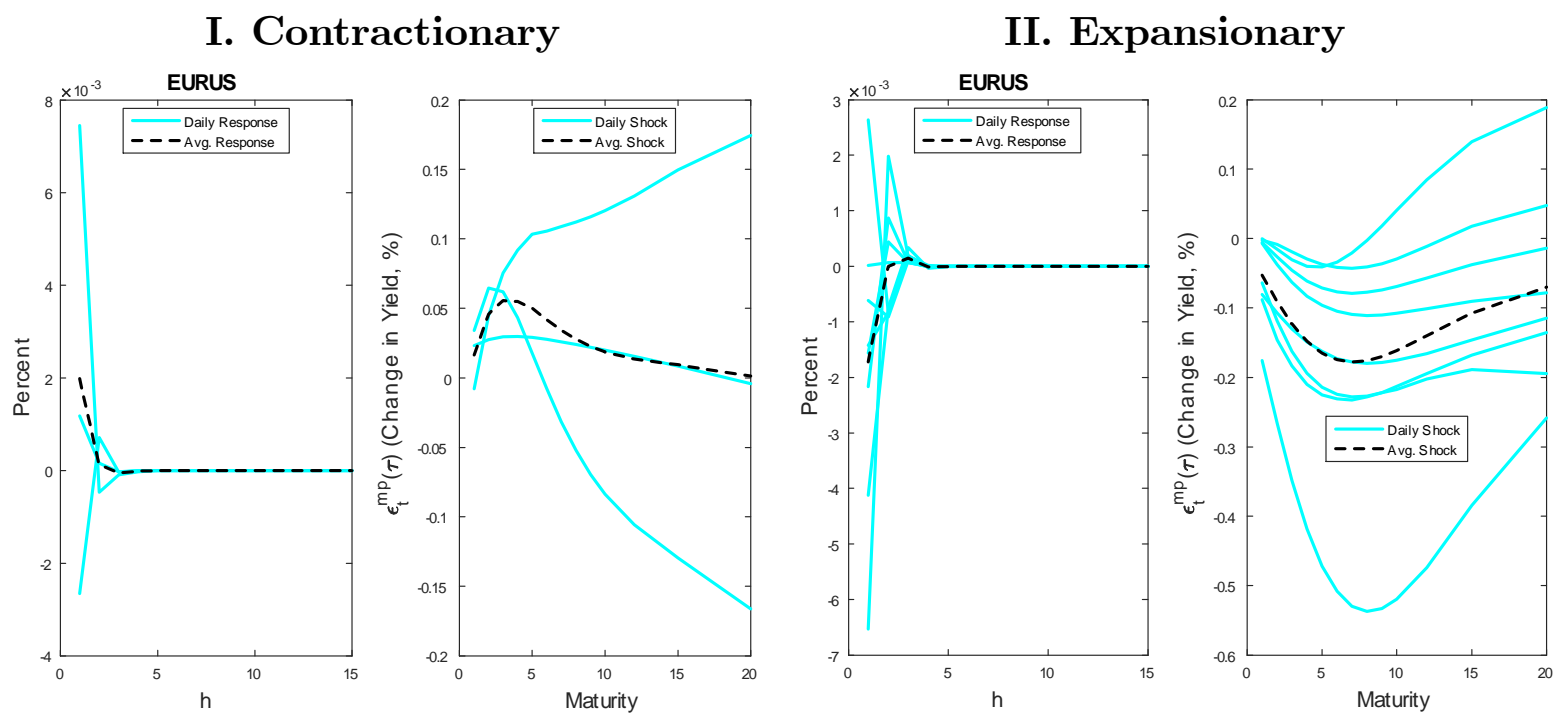

Note to the figure. Each of the four figures plots the monetary policy shock (panel on the right) and the corresponding exchange rate's response (panel on the left) for the currencies indicated in the title. "Contractionary" means $\Delta \mathcal{Y}_{5, t}^{r *}>0$ and "Expansionary" means $\Delta \mathcal{Y}_{5, t}^{r *}<0$ for all countries. 
Figure 8. Response to Relative Movements in the Relative Home vs. Foreign Real Term Structures on US Monetary Policy Announcement Dates: Unconventional Period Panel A. United Kingdom
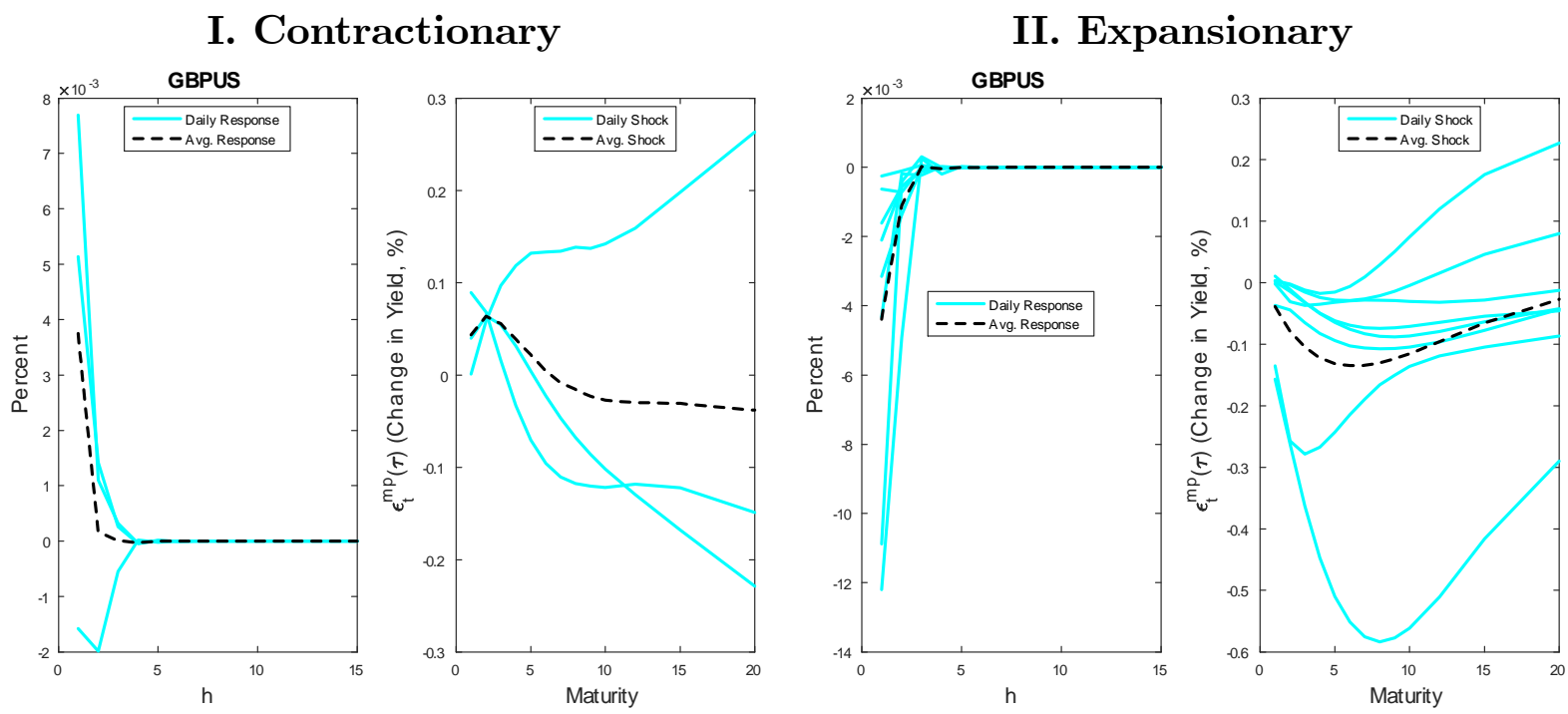

Panel A. Europe
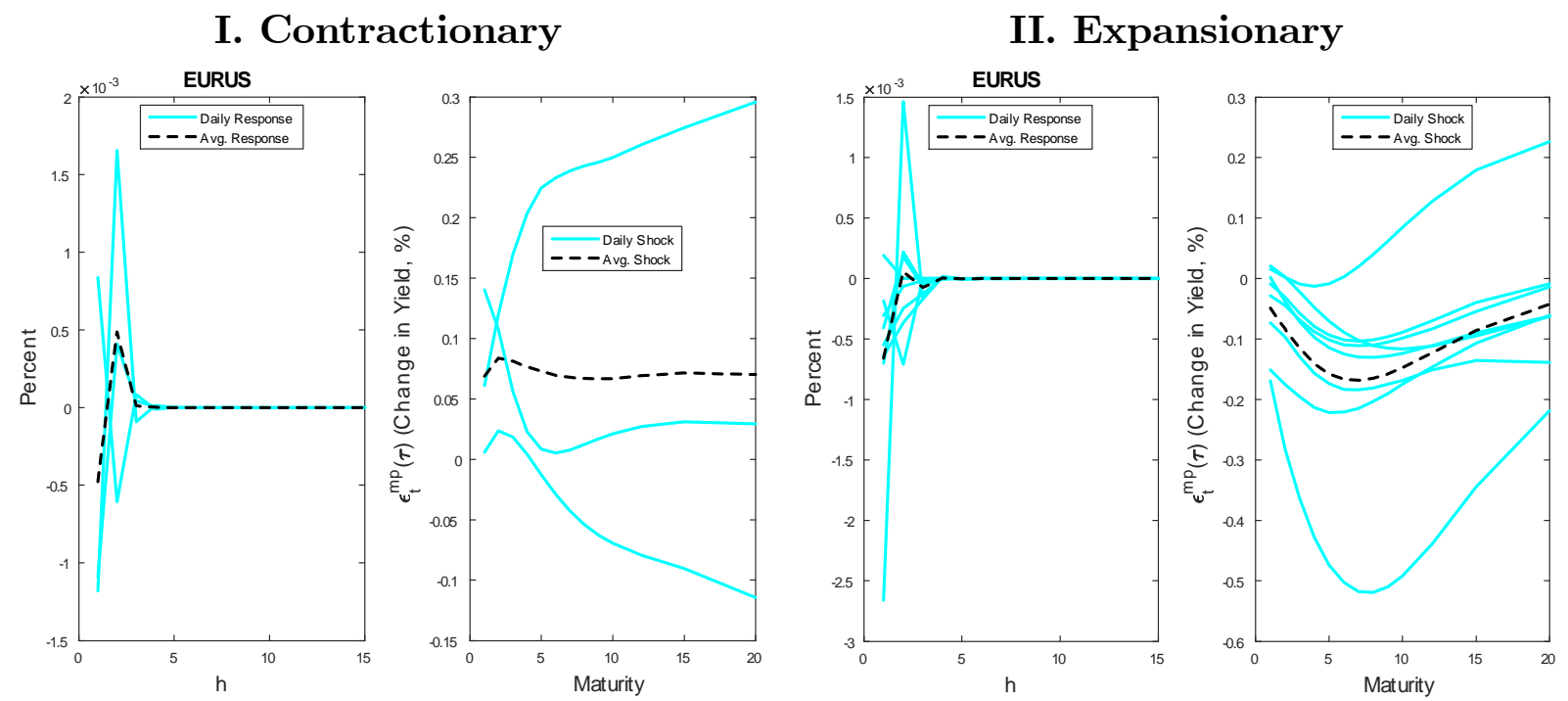

Note to the figure. Each of the four figures plots the monetary policy shock (panel on the right) and the corresponding exchange rate's response (panel on the left) for the currencies indicated in the title. "Contractionary" means $\Delta \widetilde{\mathcal{Y}}_{5, t}^{r *}>0$ and "Expansionary" means $\Delta \widetilde{\mathcal{Y}}_{5, t}^{r *}<0$ for all countries. 
Figure 9. Response Decomposition: Conventional Period, UK Panel A. United Kingdom

I. Fully Contractionary
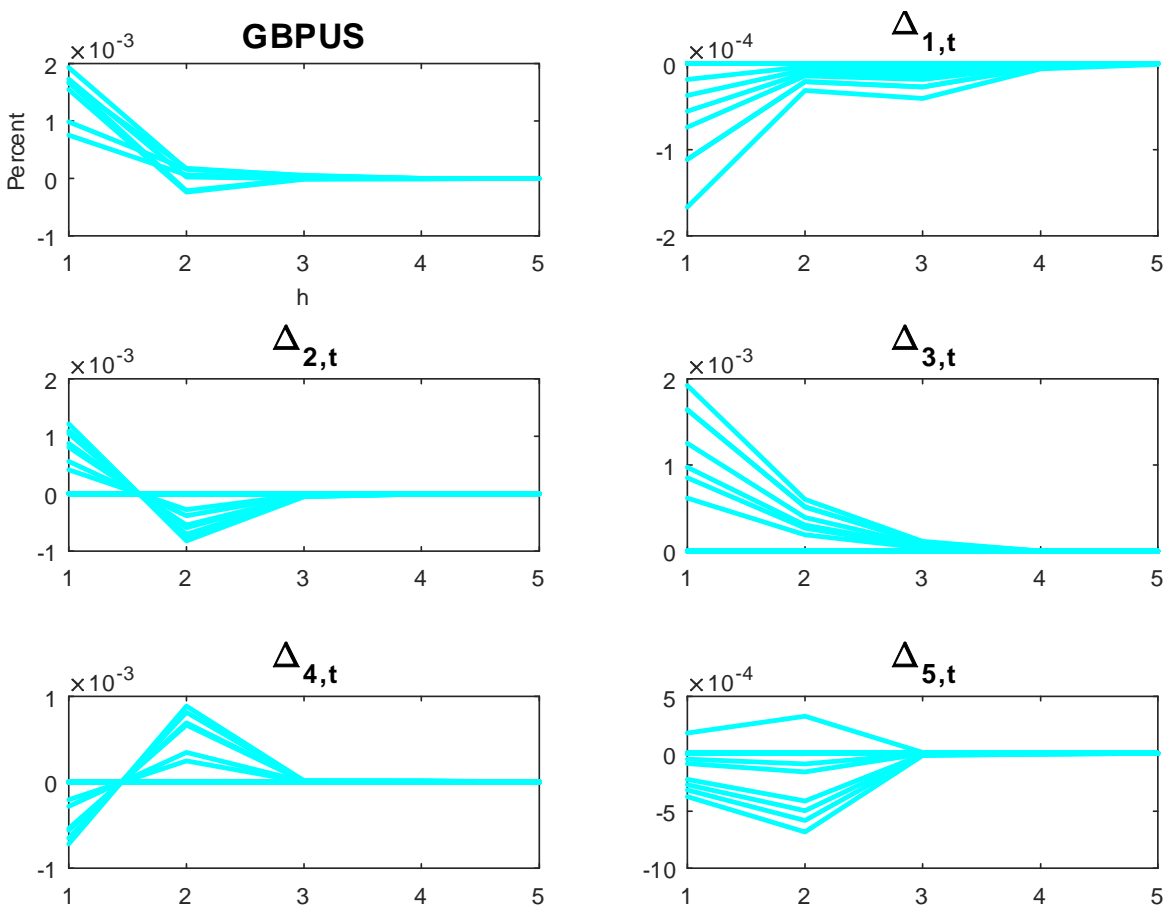

II. Fully Expansionary
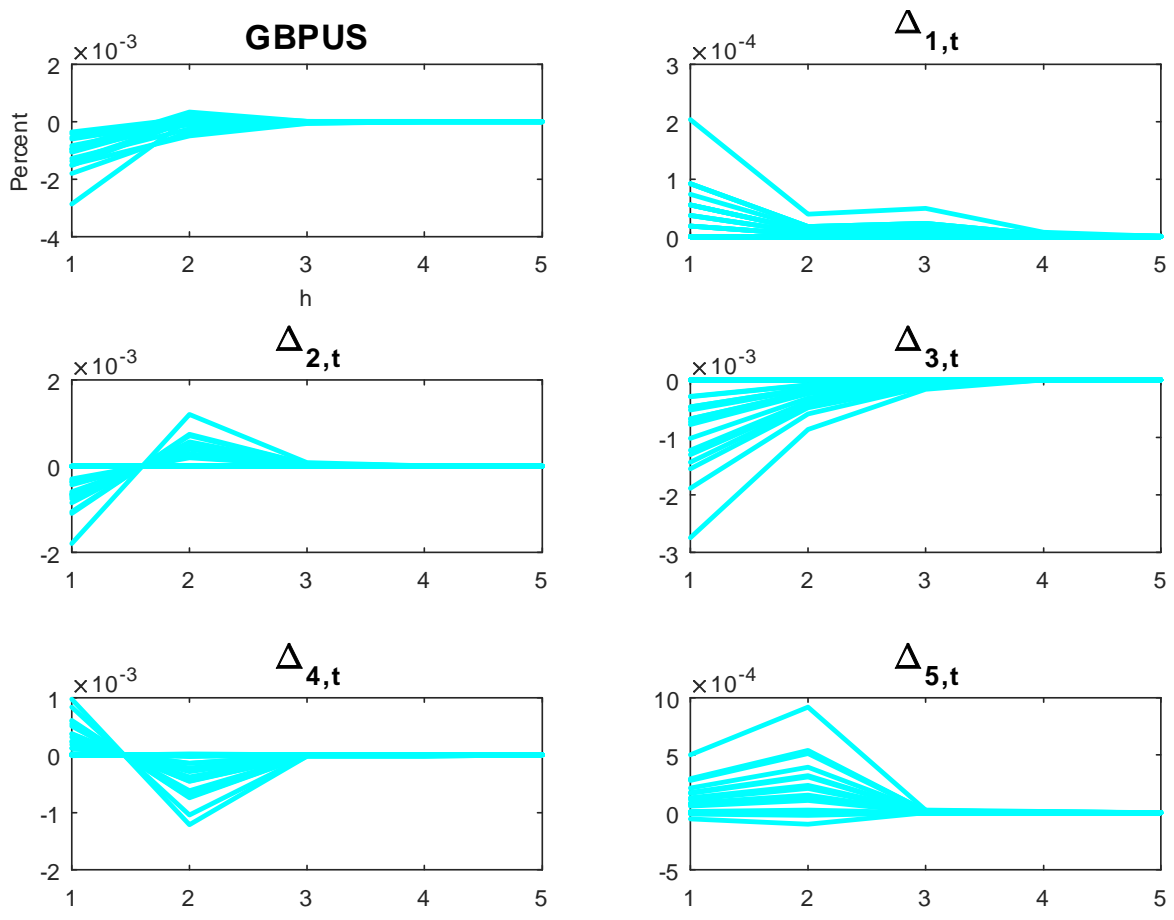

56 
Panel A (continued). United Kingdom

III. More Contractionary at Short
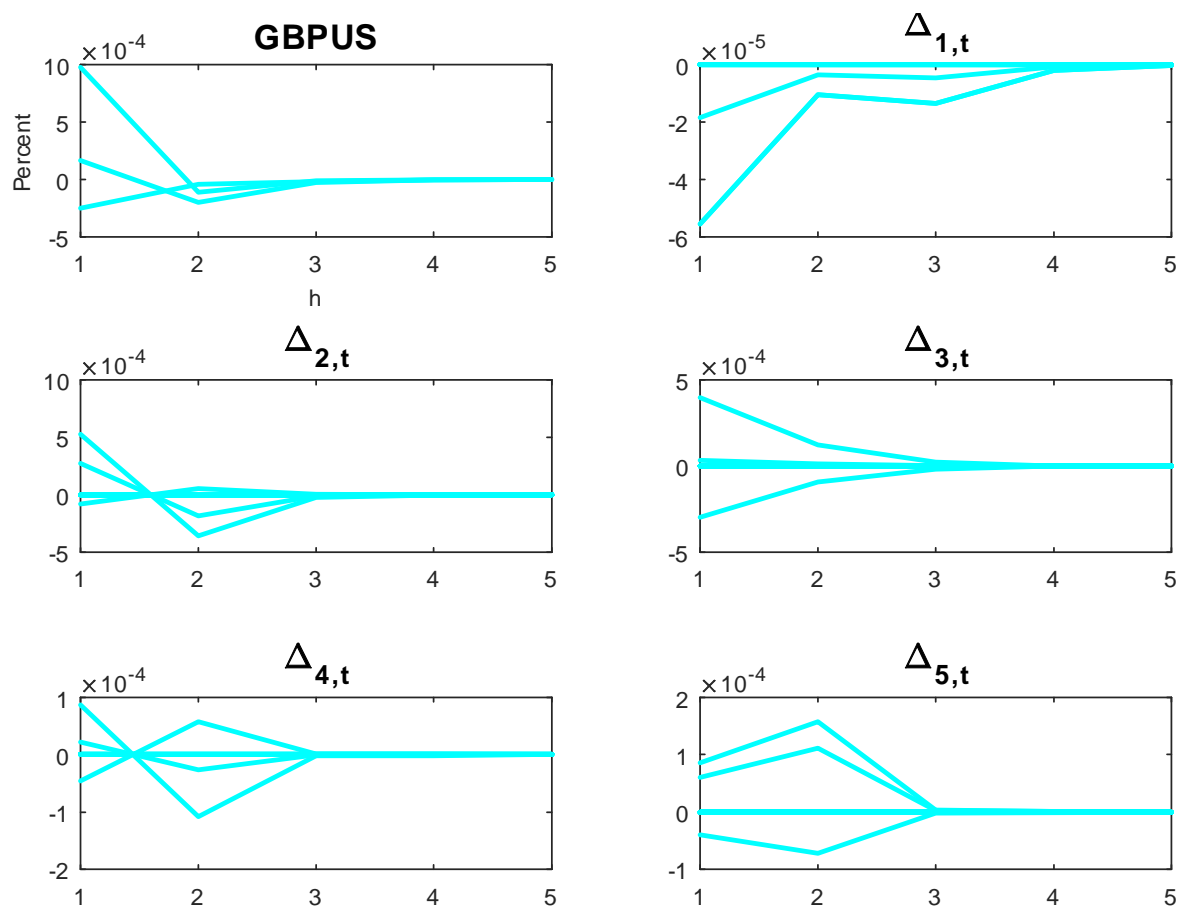

IV. More Expansionary at Short
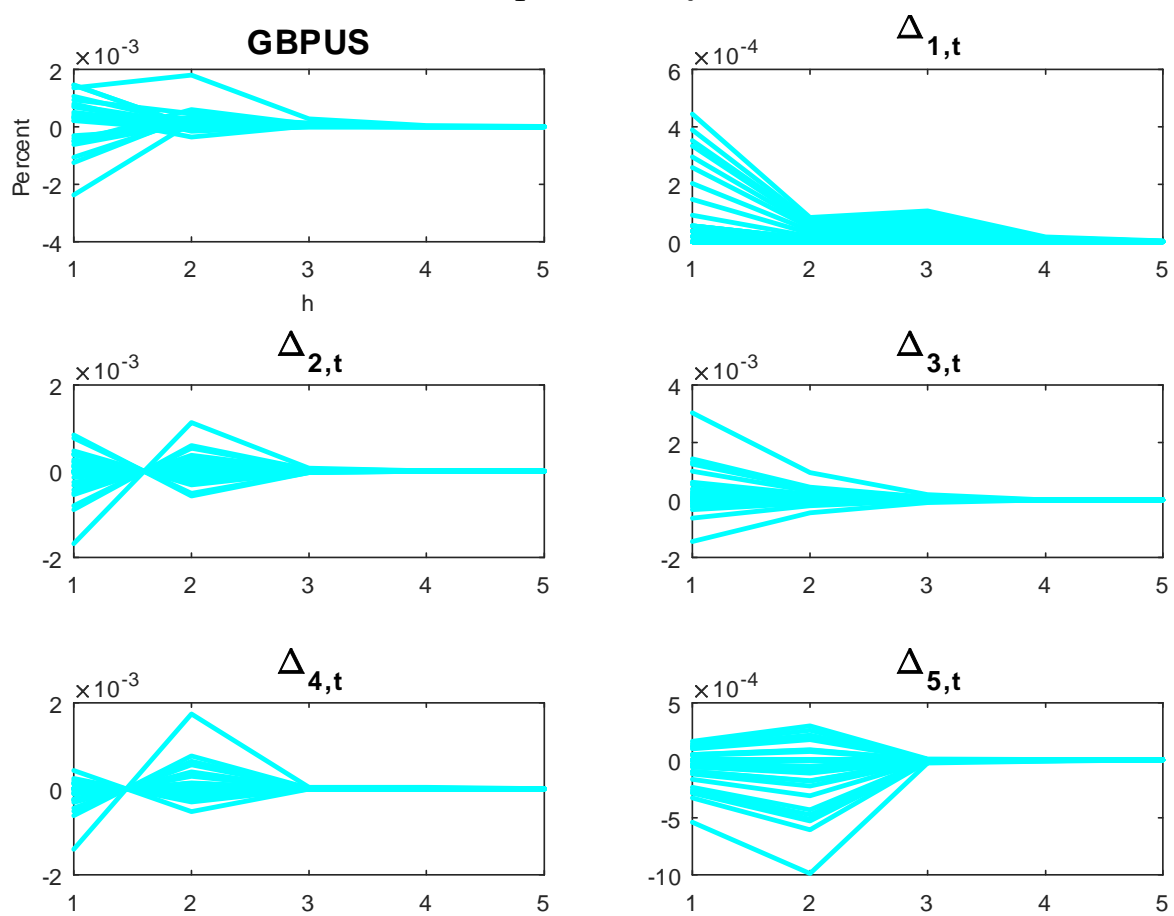
Figure 9 (continued)

\section{Panel B. Euro}

I. Fully Contractionary
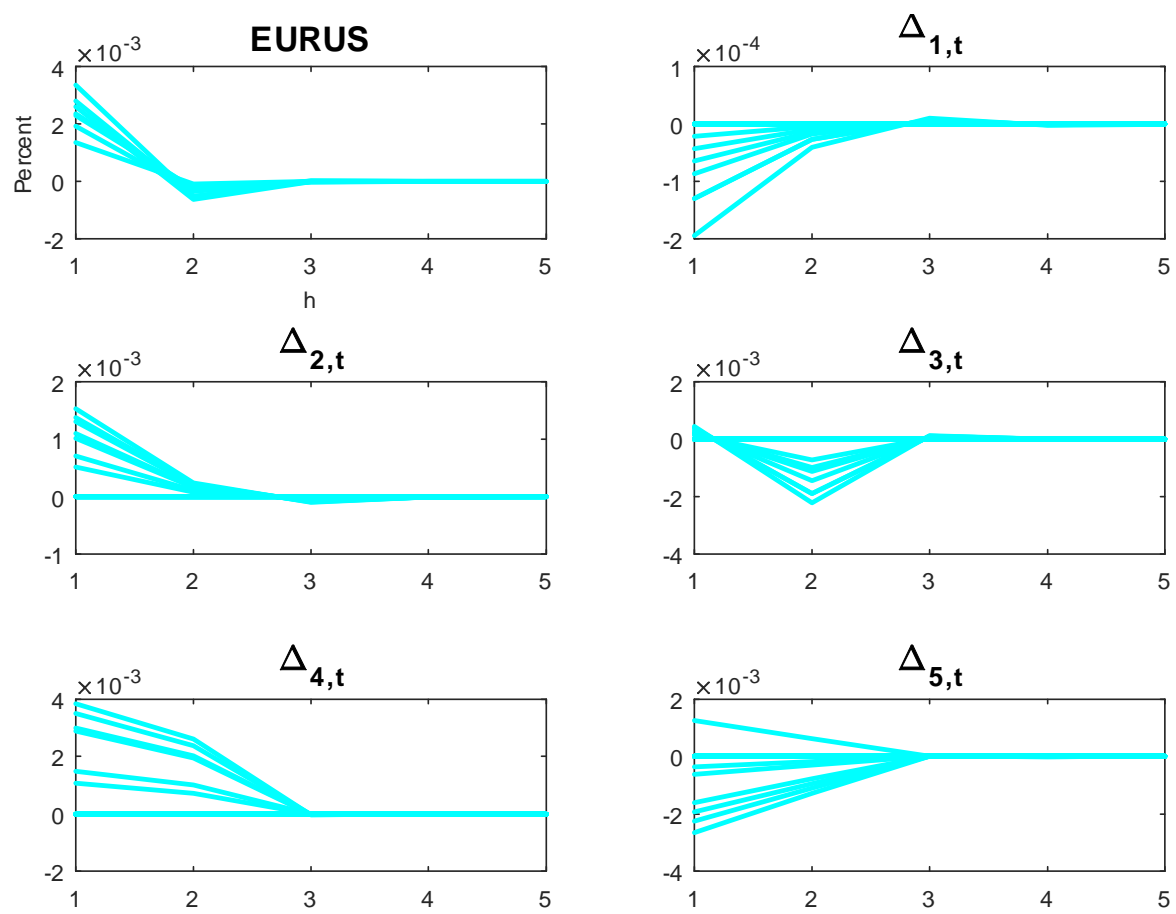

II. Fully Expansionary
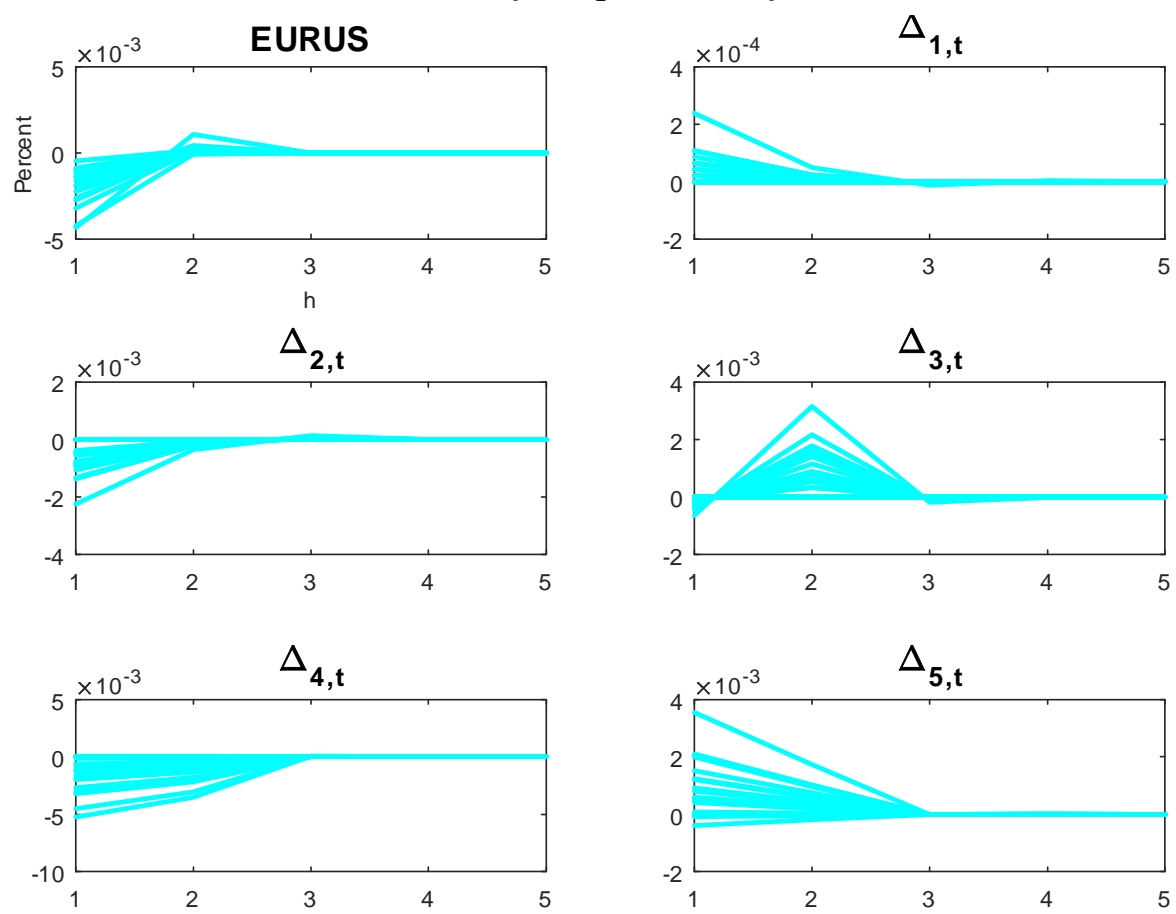


\section{Panel B (continued). Euro}

III. More Contractionary at Short
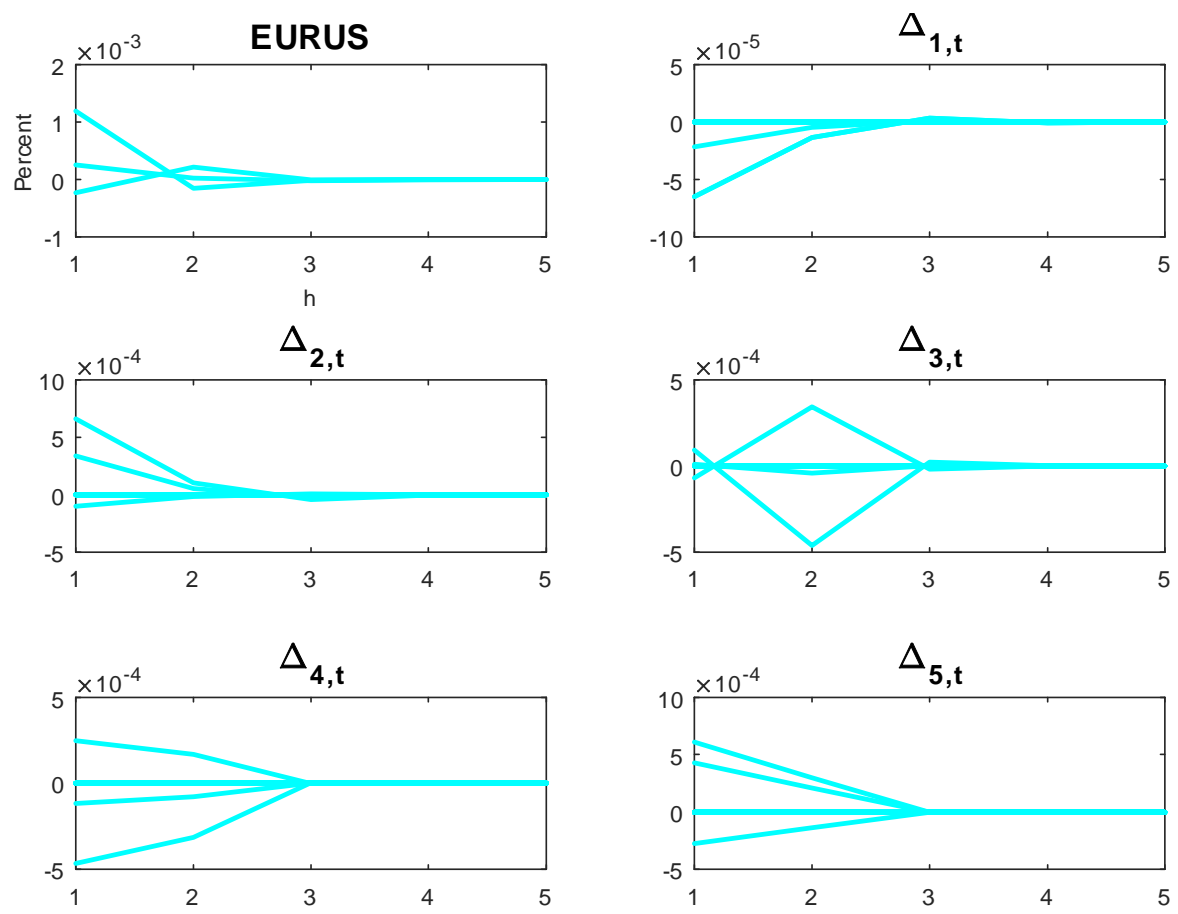

IV. More Expansionary at Short
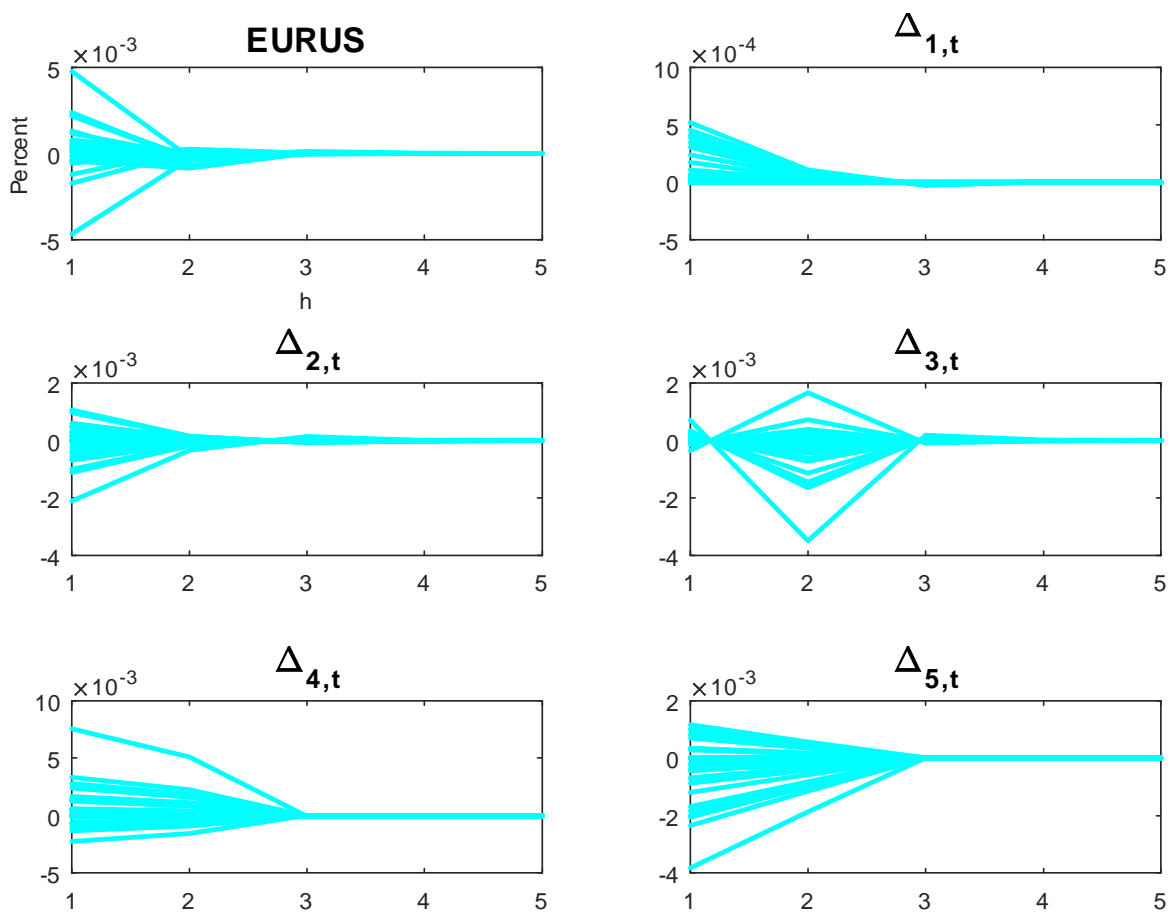
Figure 9 (continued)

Panel C. Canada

I. Fully Contractionary
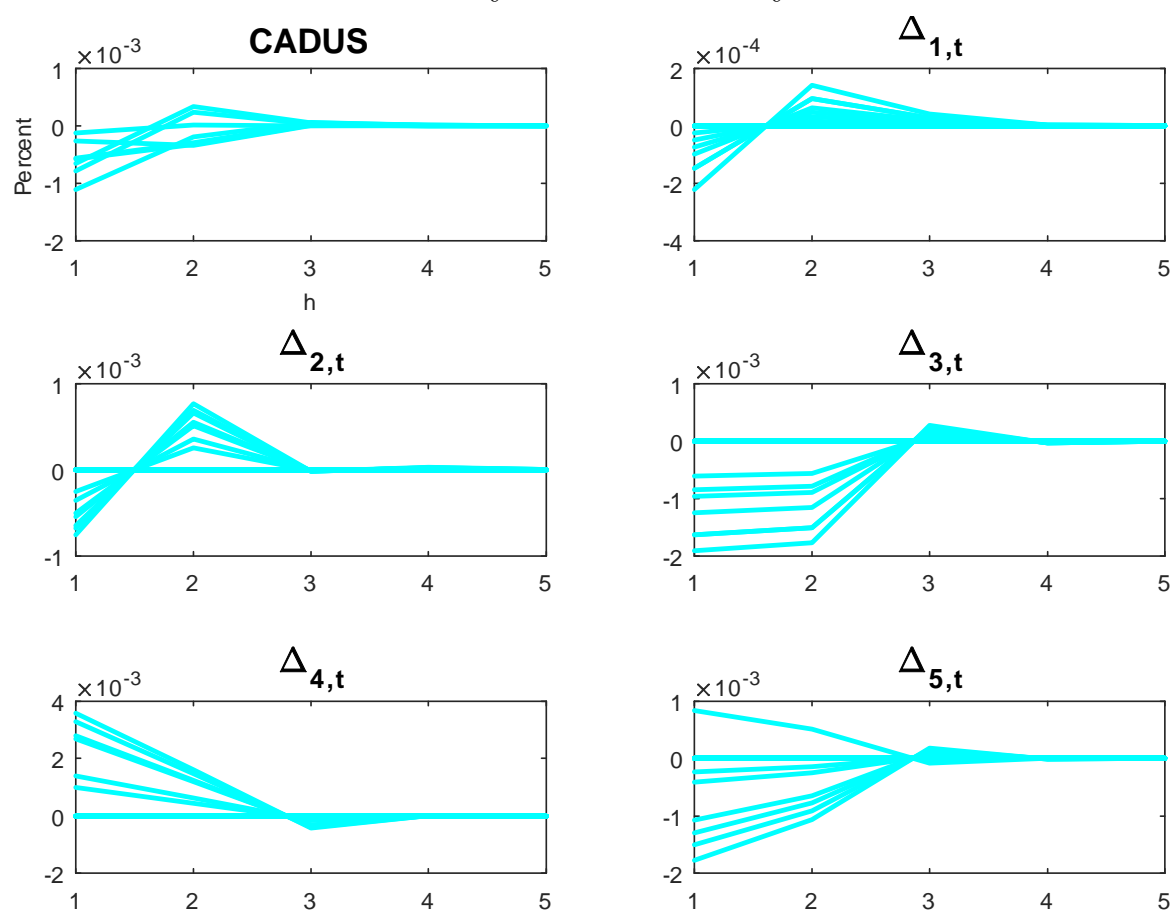

II. Fully Expansionary
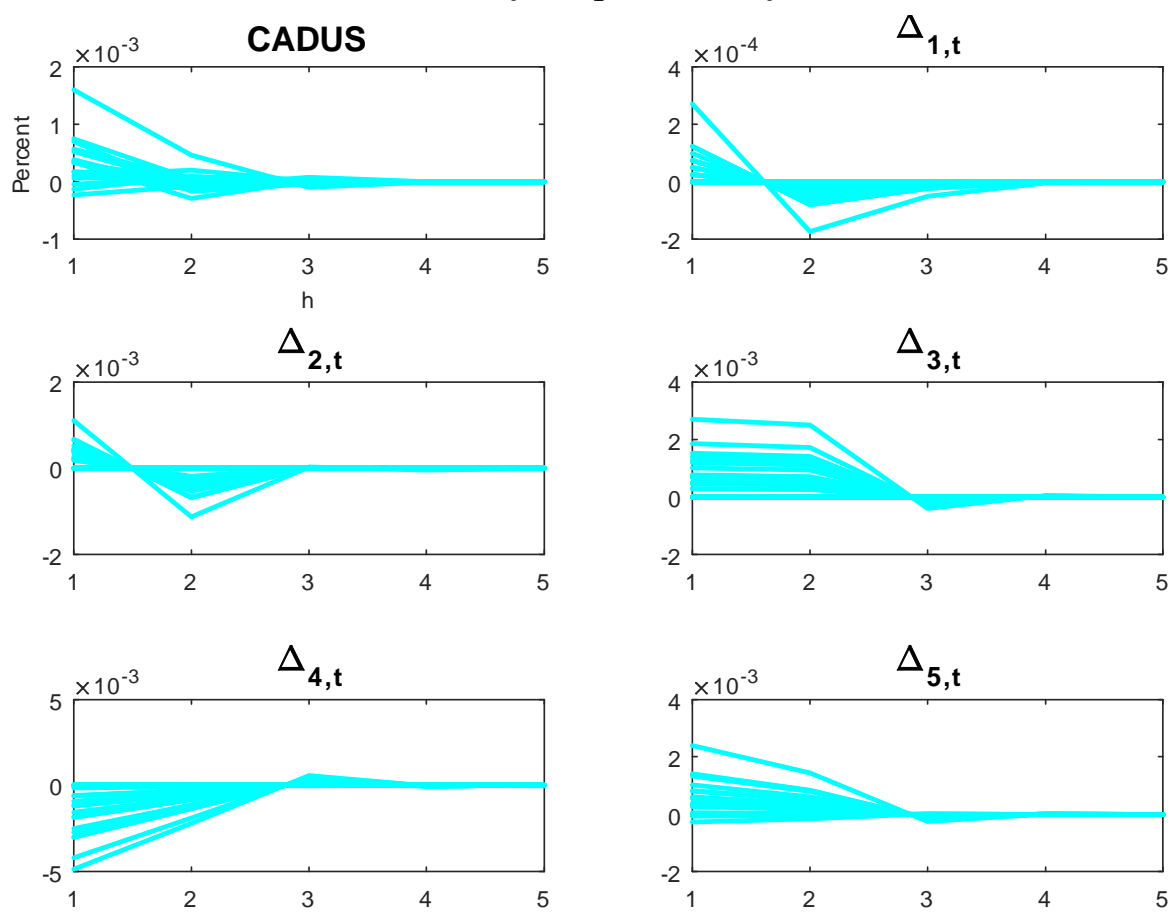
Panel C (continued). Canada

III. More Contractionary at Short
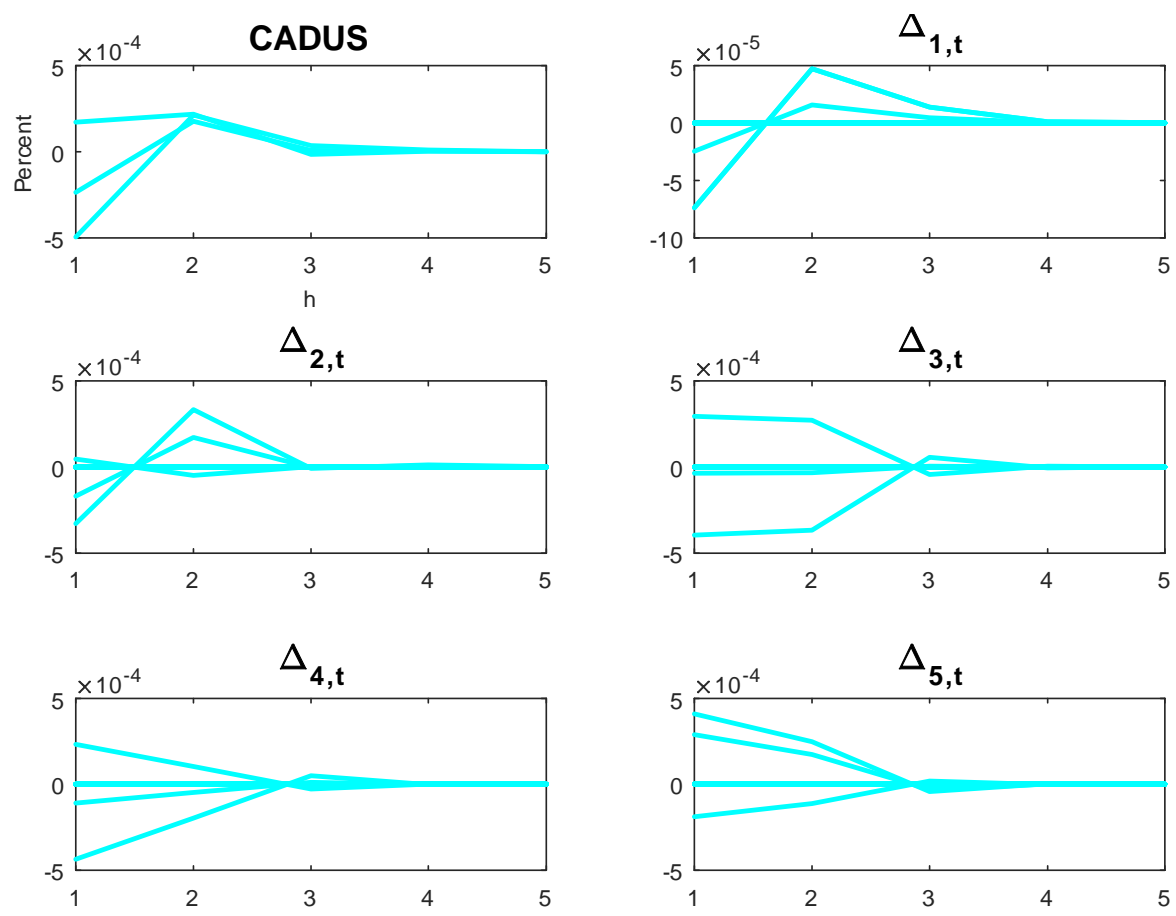

IV. More Expansionary at Short
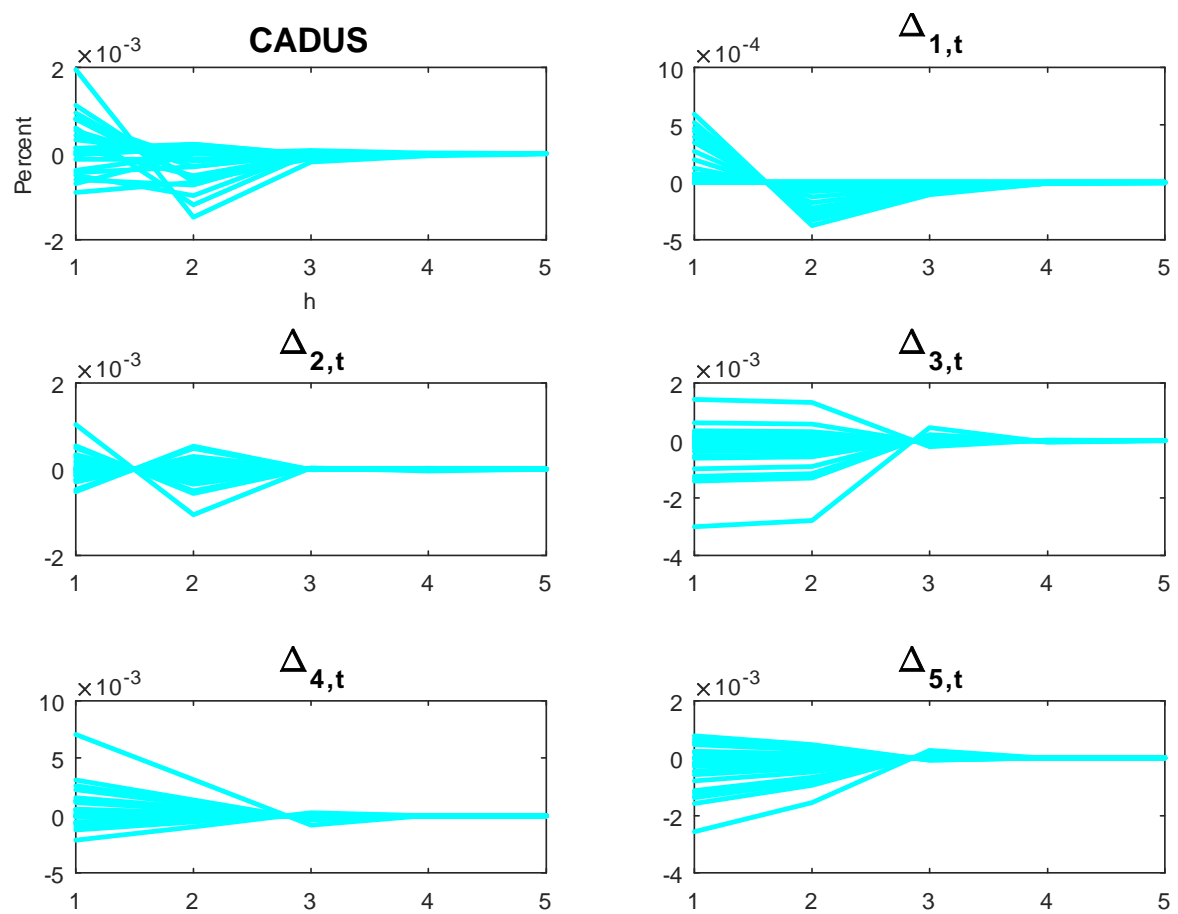
Figure 9 (continued)

Panel D. Japan

I. Fully Contractionary
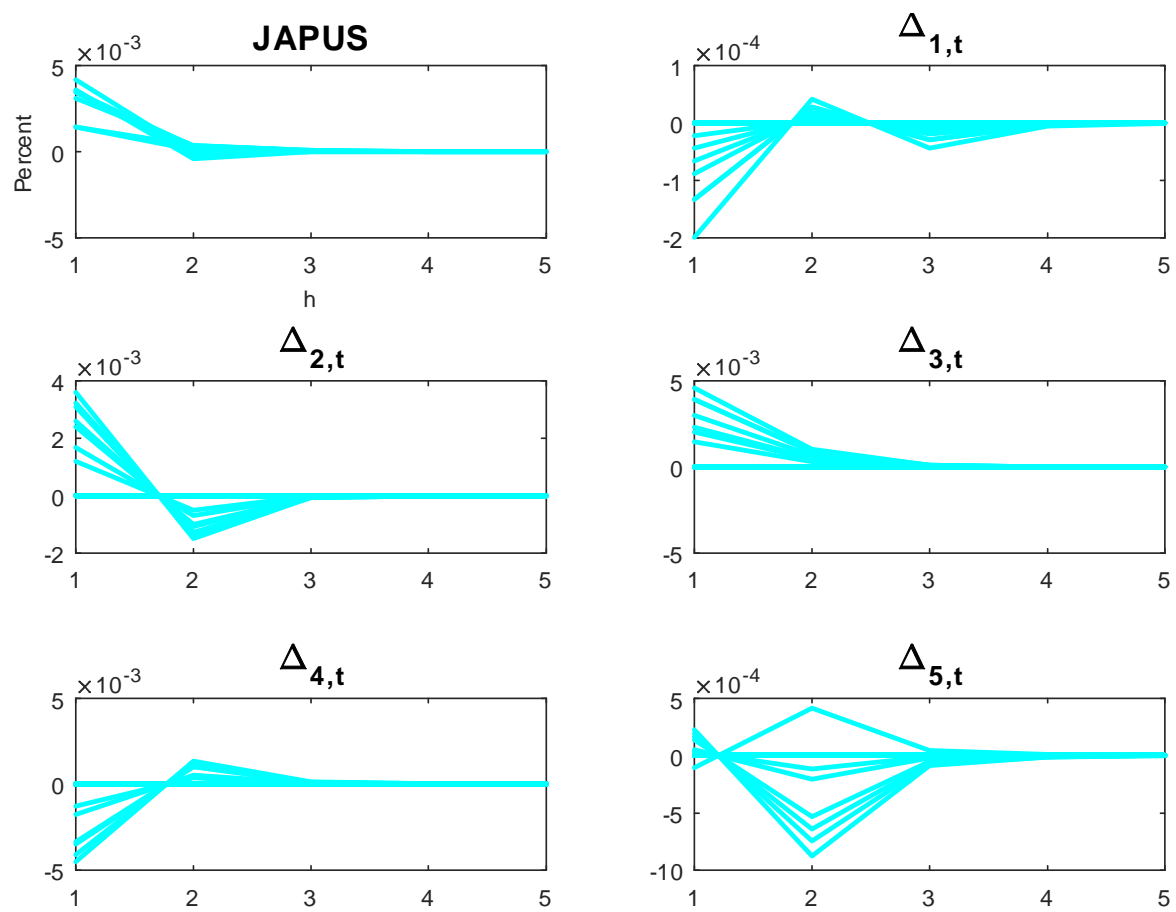

II. Fully Expansionary
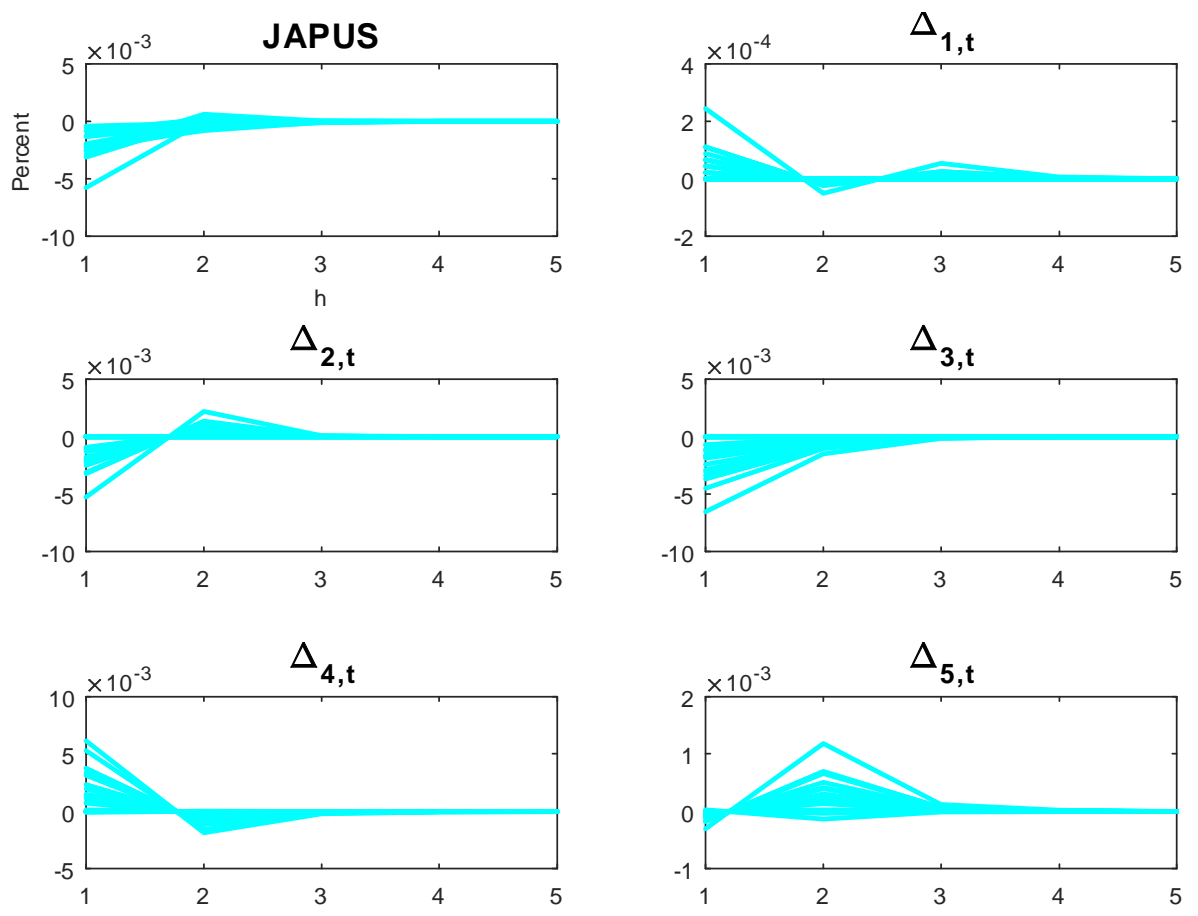


\section{Panel D (continued). Japan}

III. More Contractionary at Short
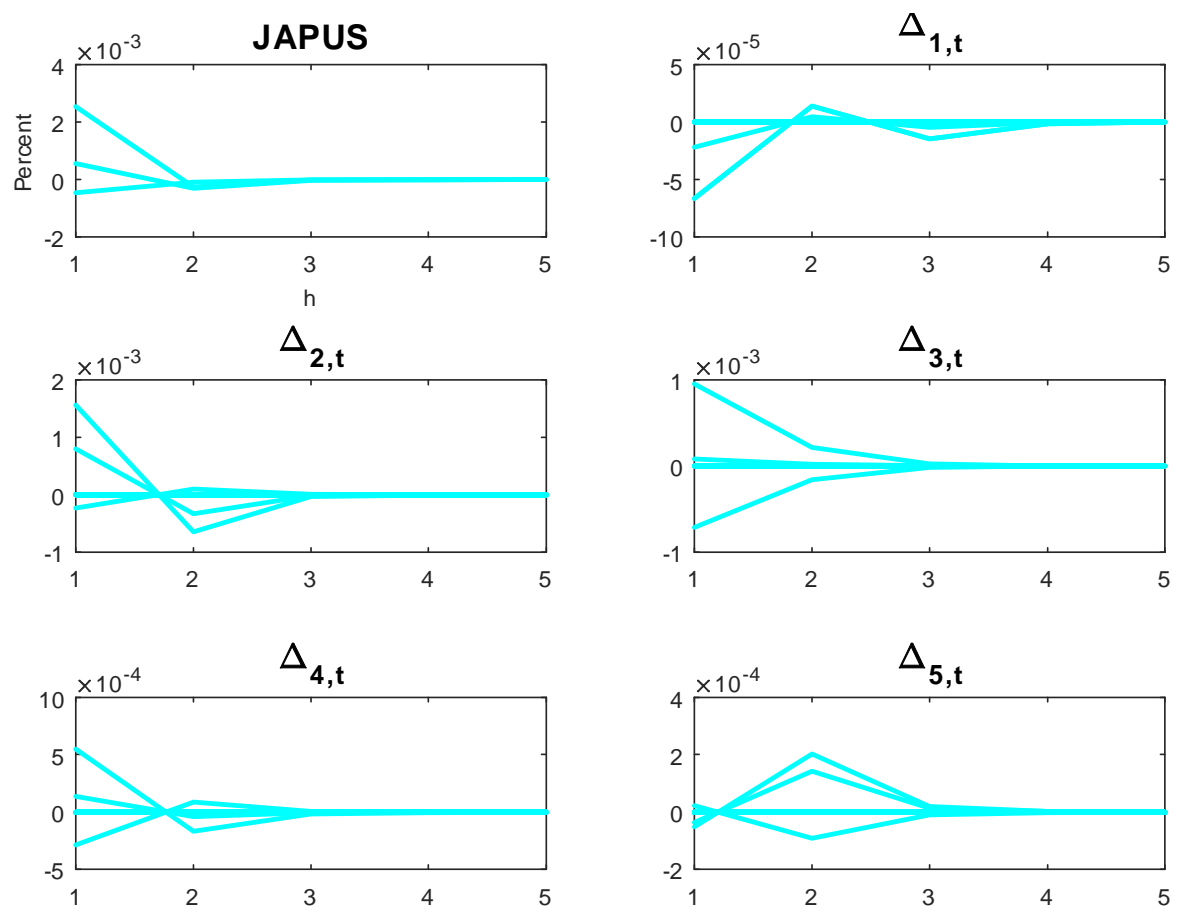

IV. More Expansionary at Short
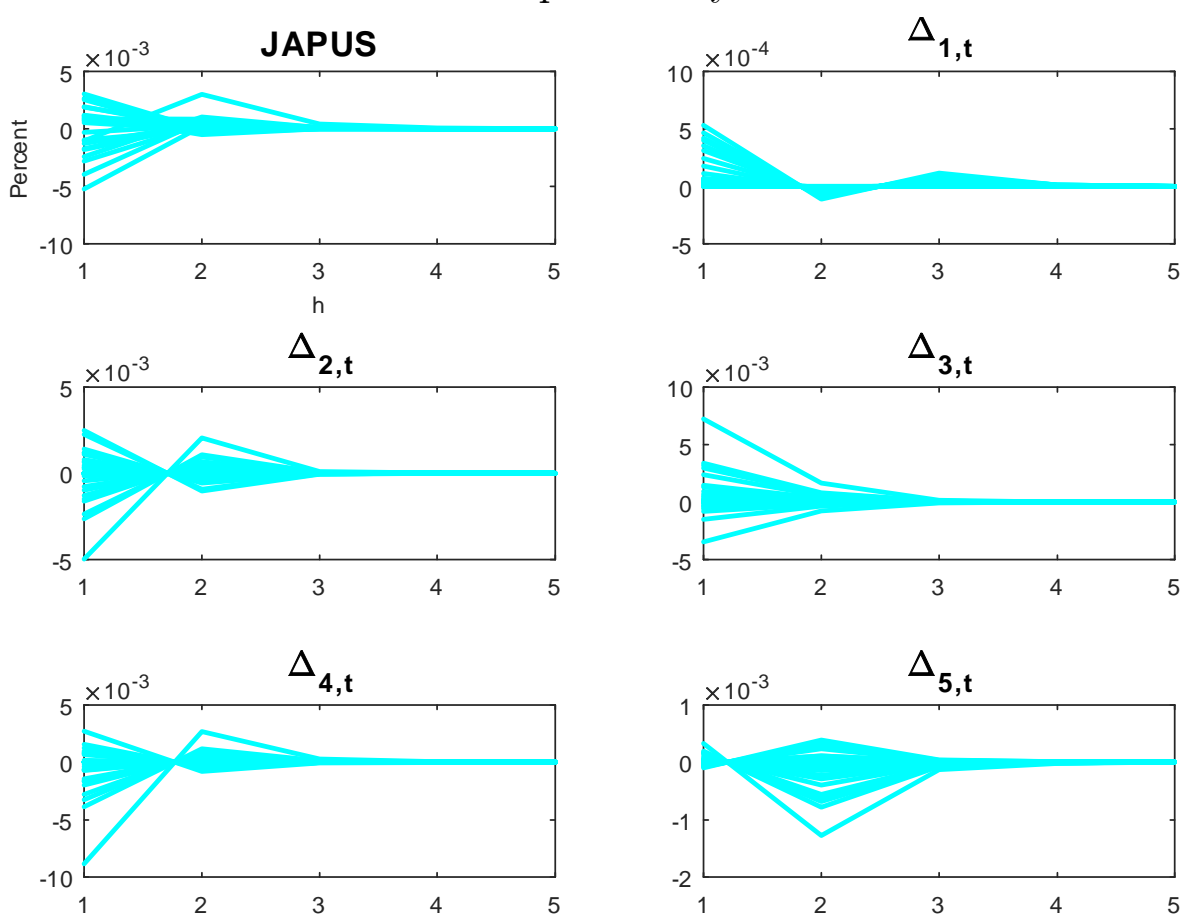
Figure 10. Response Decomposition: Unconventional Period

\section{Panel A. United Kingdom}

I. Contractionary
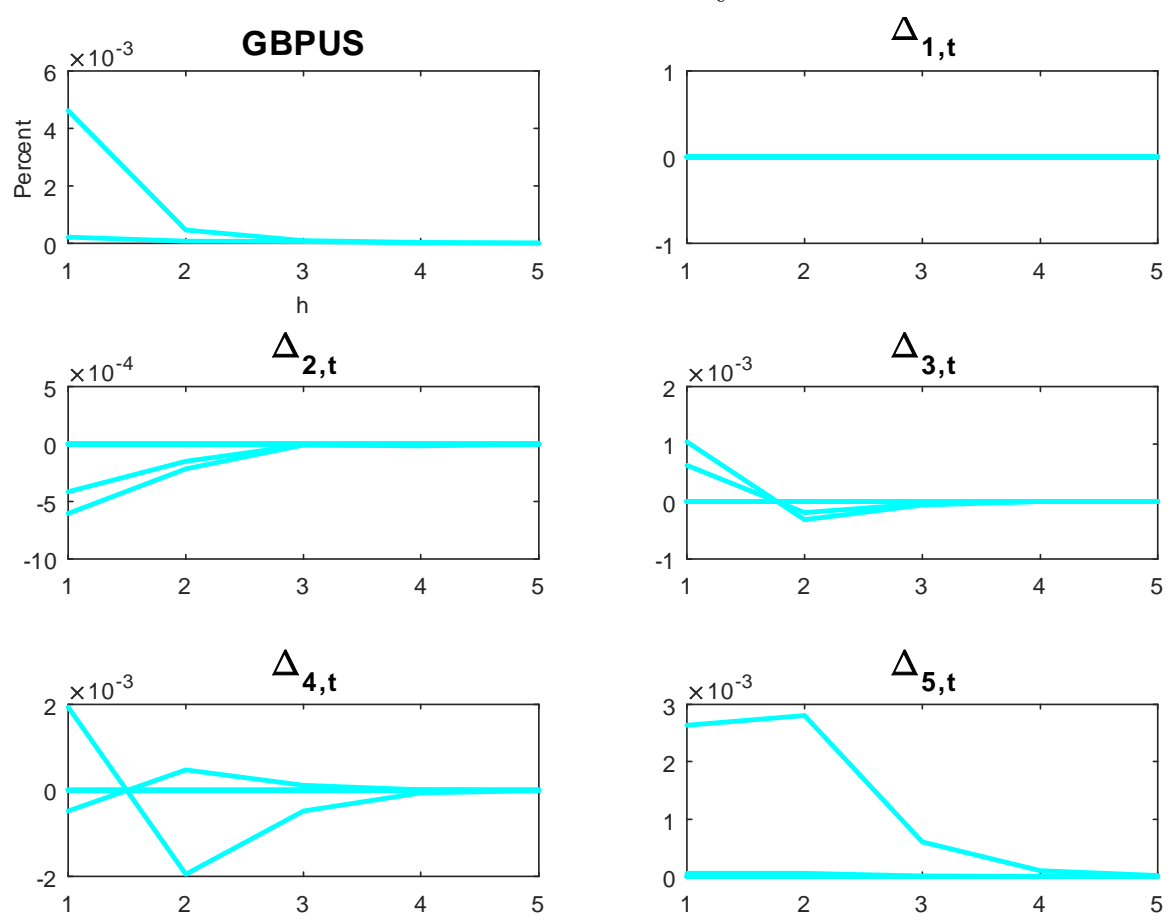

II. Expansionary
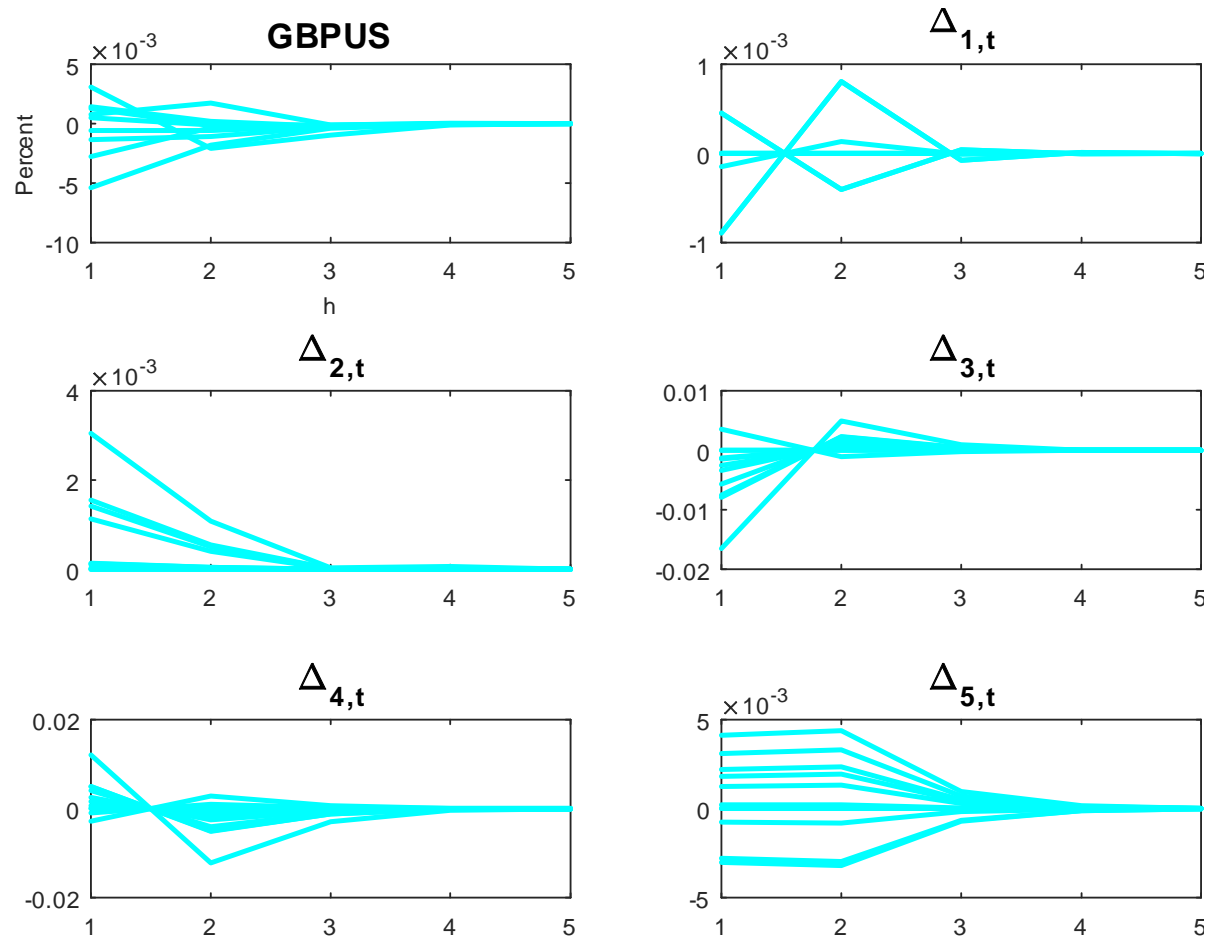


\section{Panel B. Europe}
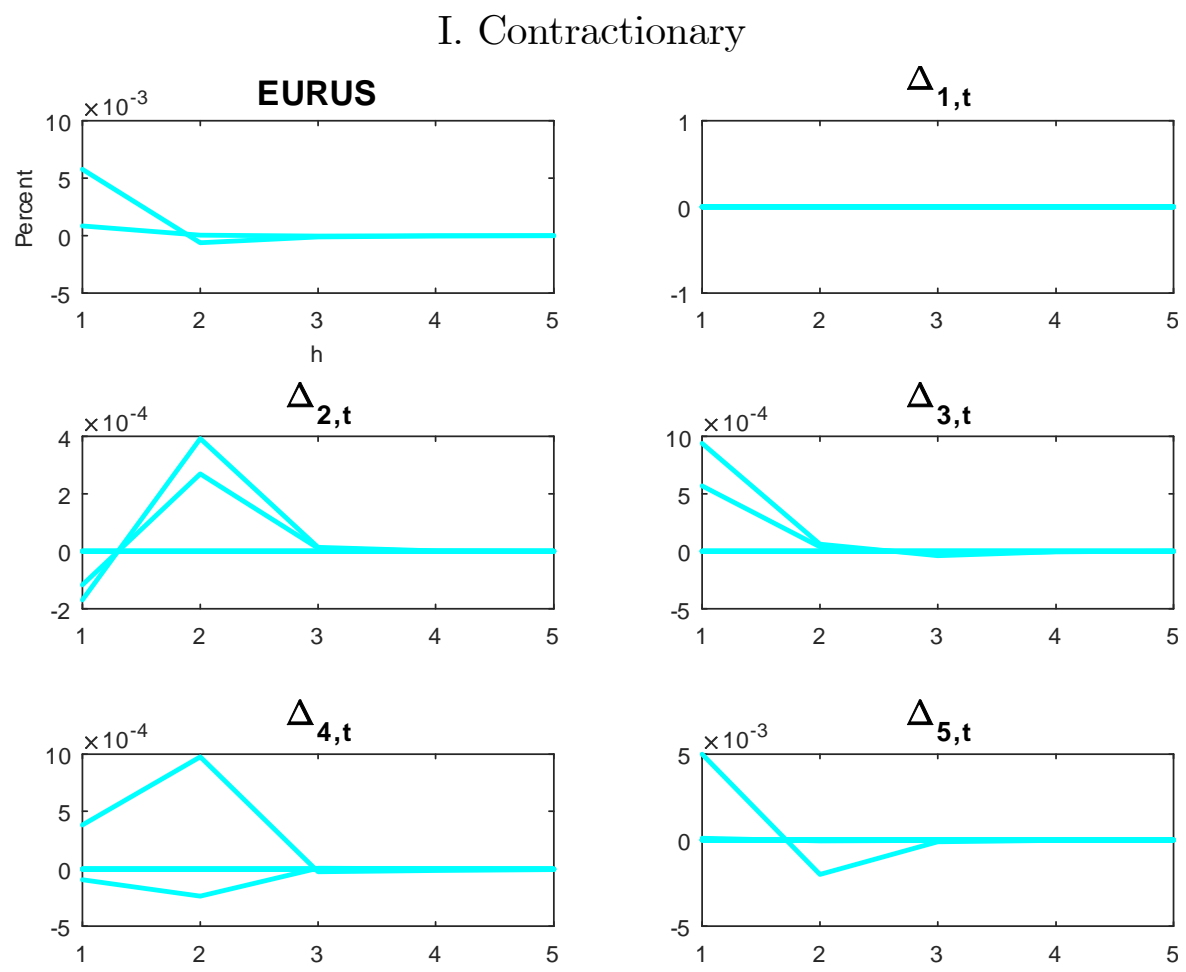

II. Expansionary
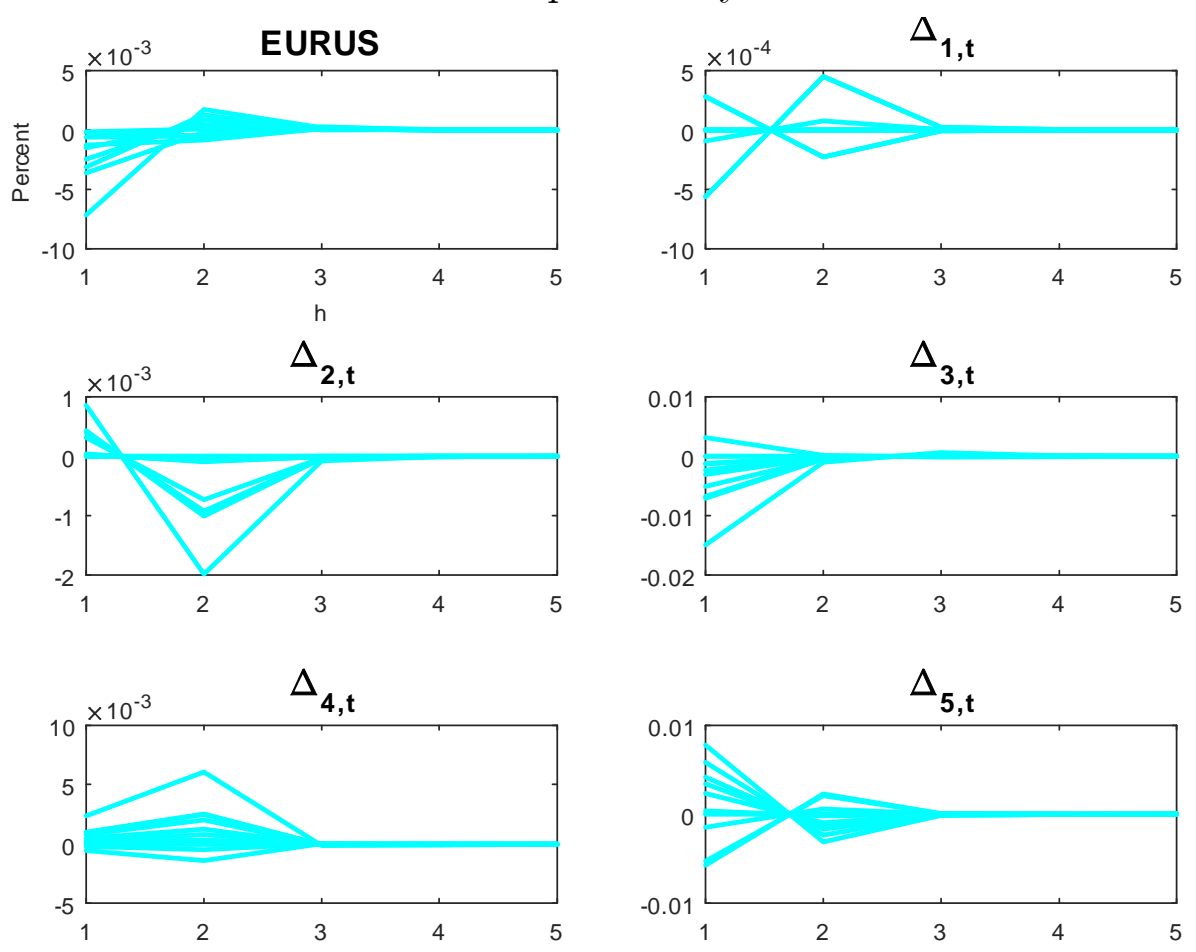
Figure 10 (continued)

\section{Panel C. Canada}

\section{Contractionary}
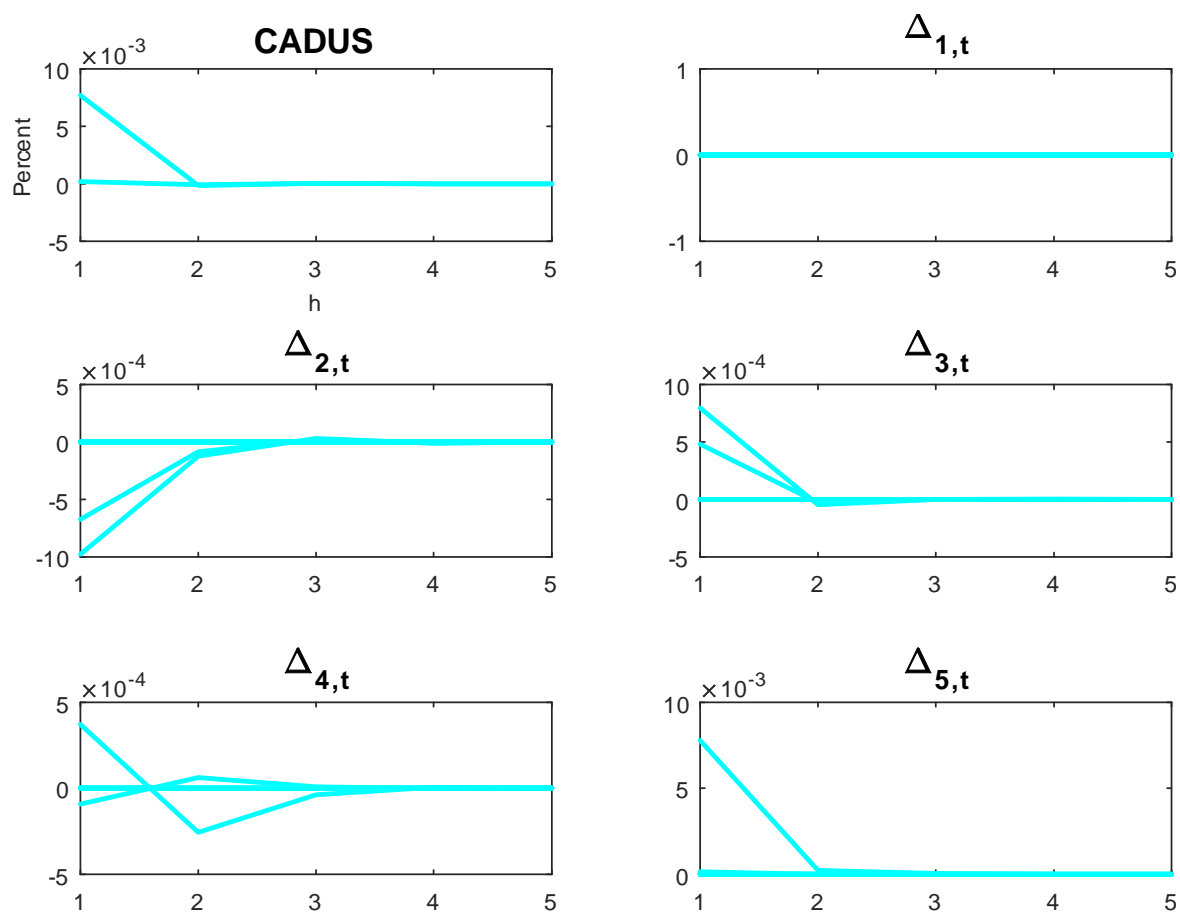

II. Expansionary
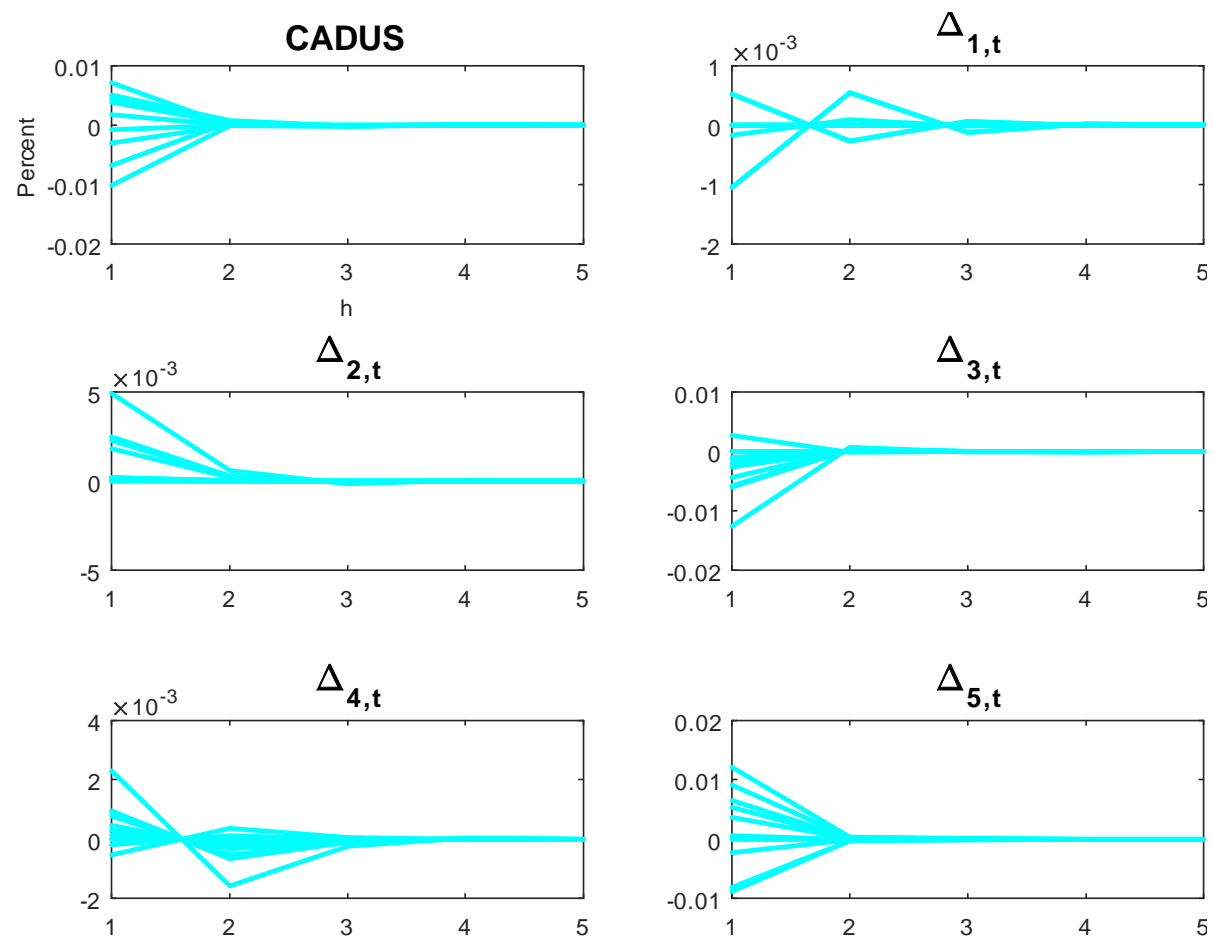


\section{Panel D. Japan}

\section{Contractionary}
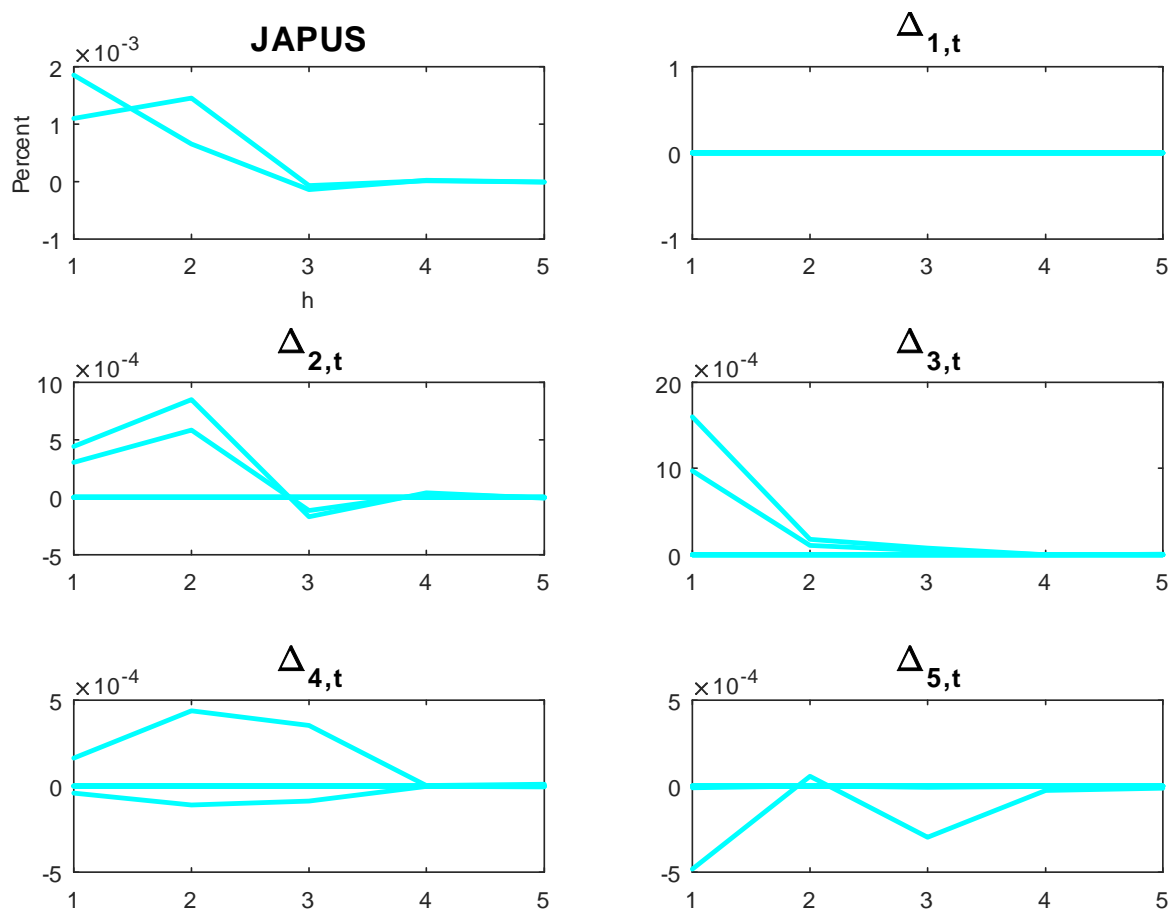

II. Expansionary
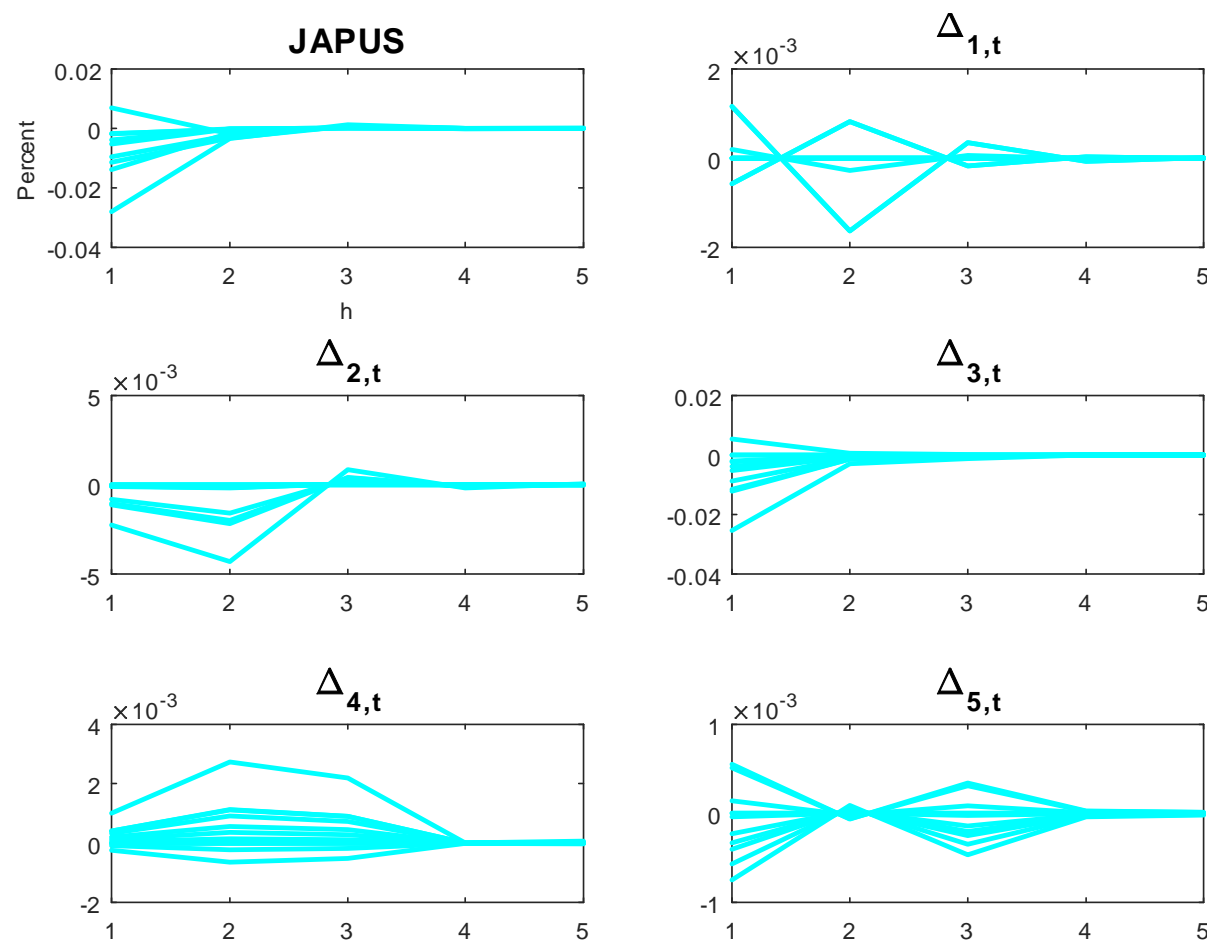
Note to Figure 9. "Fully Contractionary" means $\Delta \mathcal{Y}_{1 / 4, t}^{*}>0, \Delta \mathcal{Y}_{5, t}^{*}-\Delta \mathcal{Y}_{1 / 4, t}^{*}>0$ and "Fully Expansionary" means $\Delta \mathcal{Y}_{1 / 4, t}^{*}<0, \Delta \mathcal{Y}_{5, t}^{*}-\Delta \mathcal{Y}_{1 / 4, t}^{*}<0$. "More Contractionary at Short" means $\Delta \mathcal{Y}_{1 / 4, t}^{*}<0, \Delta \mathcal{Y}_{5, t}^{*}-\Delta \mathcal{Y}_{1 / 4, t}^{*}>0$ while "Less Expansionary at Long" means $\Delta \mathcal{Y}_{1 / 4, t}^{*}>0, \Delta \mathcal{Y}_{5, t}^{*}-\Delta \mathcal{Y}_{1 / 4, t}^{*}<0$.

Notes to Figure 10. Each of the four figures plots the monetary policy shock (panel on the right) and the corresponding exchange rate's response (panel on the left) for the currencies indicated in the title. "Contractionary" means $\Delta \mathcal{Y}_{5, t}^{*}>0$ and "Expansionary" means $\Delta \mathcal{Y}_{5, t}^{*}<0$. 